\title{
Origin of the Relative Stereoselectivity of the $\beta$-Lactam Formation in the Staudinger Reaction
}

\author{
Lei Jiao, Yong Liang, and Jiaxi $\mathrm{Xu}^{*}$ \\ Key Laboratory of Bioorganic Chemistry and Molecular Engineering of Ministry of Education, College of \\ Chemistry and Molecular Engineering, Peking University, Beijing 100871, P. R. China
}

\section{Supplemental Materials}

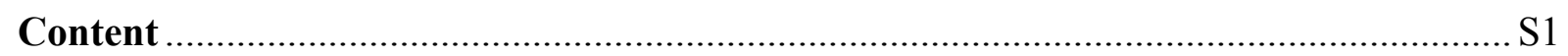

Detailed Discussion on the Isomerization of the Imine Moiety ......................................... S2

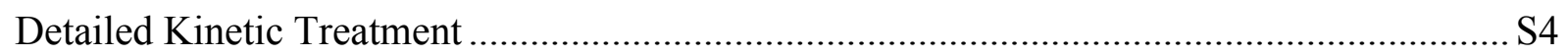

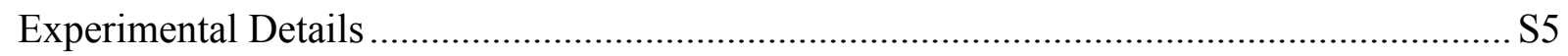

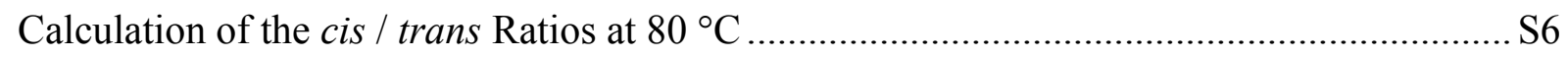

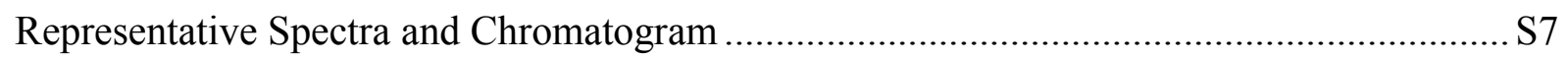

The Spectroscopic Data of the Products …...................................................................... S12

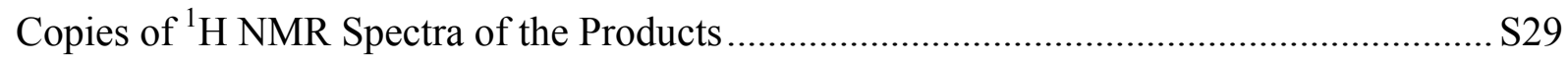




\section{Detailed Discussion on the Isomerization of the Imine Moiety}

It is unequivocal that the $\beta$-lactam products with different relative configurations are generated from zwitterionic intermediates with different configurations. To further understand how the different zwitterionic intermediates are formed, we designed and conducted the reaction of an acyl cation with an imine to simulate the formation of the zwitterionic intermediate in the Staudinger reaction. A ketene and an imine react to give a zwitterionic intermediate, while an acyl cation reacts with an imine to give an $N$-acyl iminium ion, of which imine moiety is similar to the zwitterionic intermediate and could not precede the subsequent cyclization. Thus, we can understand how the different zwitterionic intermediates are generated by analyzing experimental results.

Scheme 1. The preparation of the $N$-acetyl iminium ion $\mathbf{I}$.
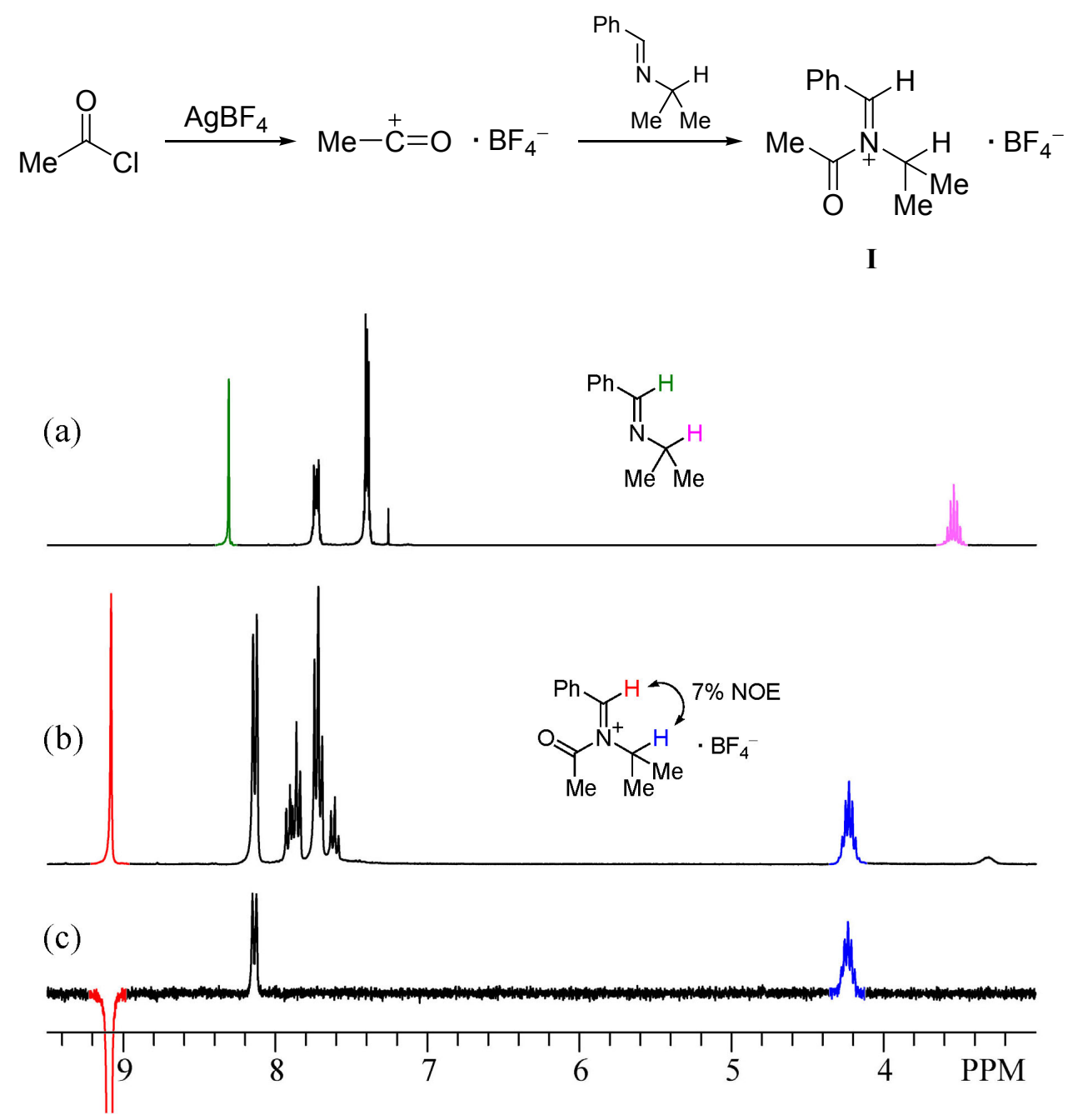

Figure 1. Partial ${ }^{1} \mathrm{H}$ NMR spectra: (a) the $N$-isopropyl imine; (b) the $N$-acetyl iminium ion I, containing small amount of benzaldehyde and $N$-isopropyl acetamide generated from the hydrolysis of $\mathbf{I}$; and (c) 1D NOE spectrum of the iminium ion $\mathbf{I}$. 
We chose acetyl cation and $N$-isopropyl imine $\mathbf{2 b}$, which mainly produces trans- $\beta$-lactam in the Staudinger reaction, as the reactants to prepare $N$-acetyl iminium I (Scheme 1). In order to avoid the nucleophilic attack of the chloride anion, ${ }^{1}$ acetyl cation was prepared by mixing equimolar amounts of acetyl chloride and $\mathrm{AgBF}_{4}$ in $\mathrm{DMSO}-d_{6}$. After separation of the precipitates of $\mathrm{AgCl}$, it was added to a solution of imine $\mathbf{2 b}$ in DMSO- $d_{6}$ to afford the $N$-acetyl iminium ion I. ${ }^{1} \mathrm{H}$ NMR spectra show that the chemical shift of the iminic hydrogen indeed shifts to lower field ${ }^{2}$ ( $\delta$ shifted from 8.31 to 9.08), which confirmed the formation of the desired $N$-acetyl iminium ion $\mathbf{I}$ (spectra $\mathbf{a}$ and $\mathbf{b}$ in Figure 1). The NOE experiment indicates that the iminium ion $\mathbf{I}$ only exists in $E$ configuration (spectrum $\mathbf{c}$ in Figure 1). The prepared iminium ion I was heated to $80{ }^{\circ} \mathrm{C}$ to observe whether the thermal isomerization occurred. However, after maintained at such temperature for several tens of minutes, I gradually became messy and the iminium ion with $Z$ configuration was never detected.

The above results reveal that the imine moiety in the $N$-acetyl iminium ion did not isomerize though its $\mathrm{C}=\mathrm{N}$ double bond becomes weaker, and also demonstrate that the isomerization of the imine itself hardly occurs under normal reaction conditions due to its higher bond order. Considering that the imine moiety, at least in part, indeed isomerizes in the Staudinger reaction, we believe that the isomerization of the zwitterionic intermediate only occurs during the direct ring-closure step. From geometry viewpoint, the isomerization progress can be explained as follows: the theoretical investigation reveals that the stable conformation of the zwitterionic intermediate is s-trans $\mathbf{I I}$, but the intermediate must adopt a near planar $s$-cis configuration IIIa to facilitate cyclization; ${ }^{3}$ the isomerization occurring during the ring-closure process can release the steric hindrance of the s-cis configuration IIIa to form a less bulky intermediate IIIb (Figure 2).

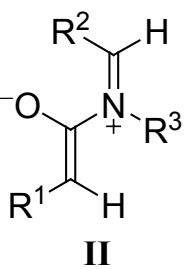

s-trans<smiles>[R]C=[N+]([R])C([O-])=C([R])[R]</smiles>

IIIa<smiles>[R]/C=[R](\[R])N([R])/C([O-])=C\[R]</smiles>

IIIb

Figure 2. The configurations of the zwitterionic intermediates.

\footnotetext{
${ }^{1}$ Bose et al studied the NMR spectrum of a $\mathrm{CCl}_{4}$ solution containing equimolar amounts of acetyl chloride and benzylideneaniline and found that $N$-[chloro(phenyl)methyl]- $N$-phenylacetamide, instead of $N$-acetyl iminium ion, was generated (the chemical shift of the iminic hydrogen shifted from 8.38 to 7.90). See: Bose, A. K.; Spiegelman, G.; Manhas, M. S. Tetrahedron Lett. 1971, 12, 3167-3170.

2 Olah, G. A.; Kreienbuhl, P. J. Am. Chem. Soc. 1967, 89, 4756-4759.

3 Cossio, F. P.; Ugalde, J. M.; Lopez, X.; Lecea, B.; Palomo, C. J. Am. Chem. Soc., 1993, 115, 995-1004.
} 


\section{Detailed Kinetic Treatment}<smiles>[R]C=CC=O</smiles>

A<smiles>[R]C=N</smiles>

B<smiles>c1ccccc1</smiles><smiles></smiles>

C

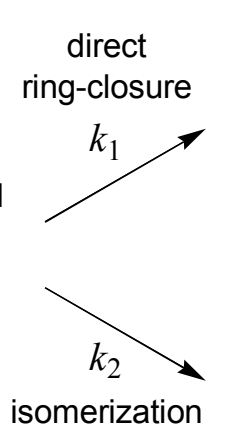<smiles>[R]C1C(=O)N([R])C1[R]</smiles>

cis- $\beta$-lactam

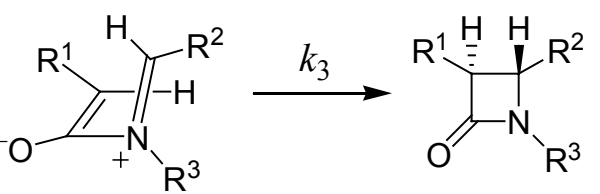

trans- $\beta$-lactam

According to the above kinetic system, the rate law of each reaction is:

$$
\begin{aligned}
& \frac{\mathrm{d}[\text { cis }]}{\mathrm{d} t}=k_{1}[\mathrm{C}] \\
& \frac{\mathrm{d}[\text { trans }]}{\mathrm{d} t}=k_{3}[\mathrm{D}] \\
& \frac{\mathrm{d}[\mathrm{D}]}{\mathrm{d} t}=k_{2}[\mathrm{C}]-k_{3}[\mathrm{D}] \\
& \frac{\mathrm{d}[\mathrm{C}]}{\mathrm{d} t}=k_{\mathrm{a}}[\mathrm{A}][\mathrm{B}]-k_{1}[\mathrm{C}]-k_{2}[\mathrm{C}]-k_{\mathrm{d}}[\mathrm{C}] \\
& \frac{\mathrm{d}[\mathrm{A}]}{\mathrm{d} t}=k_{\mathrm{d}}[\mathrm{C}]-k_{\mathrm{a}}[\mathrm{A}][\mathrm{B}] \\
& \frac{\mathrm{d}[\mathrm{B}]}{\mathrm{d} t}=k_{\mathrm{d}}[\mathrm{C}]-k_{\mathrm{a}}[\mathrm{A}][\mathrm{B}]
\end{aligned}
$$

Integrating both sides of the equations (1), (2) and (3) gives:

$$
\begin{aligned}
& {[\text { cis }]=k_{1} \int[\mathrm{C}] \mathrm{d} t} \\
& {[\text { trans }]=k_{3} \int[\mathrm{D}] \mathrm{d} t} \\
& {[\mathrm{D}]=k_{2} \int[\mathrm{C}] \mathrm{d} t-k_{3} \int[\mathrm{D}] \mathrm{d} t \quad \Rightarrow \quad k_{3} \int[\mathrm{D}] \mathrm{d} t=k_{2} \int[\mathrm{C}] \mathrm{d} t-[\mathrm{D}]}
\end{aligned}
$$

Thus, the ratio of cis and trans isomers could be described as follows:

$$
\frac{[\text { cis }]}{[\text { trans }]}=\frac{k_{1} \int[\mathrm{C}] \mathrm{d} t}{k_{3} \int[\mathrm{D}] \mathrm{d} t}=\frac{k_{1} \int[\mathrm{C}] \mathrm{d} t}{k_{2} \int[\mathrm{C}] \mathrm{d} t-[\mathrm{D}]}
$$

Because the cyclization step is very fast, the concentration of the intermediate $\mathbf{D}$ is very low, i.e. $[\mathrm{D}] \rightarrow 0$, thus:

$$
\frac{[\text { cis }]}{[\operatorname{trans}]}=\frac{k_{1}}{k_{2}}
$$




\section{Experimental Details}

General. $\alpha$-Diazocarbonyl compounds $\mathbf{1},{ }^{4} \mathbf{1 0 a}-\mathbf{f},{ }^{5}$ and ethyl 2-diazo-3-oxo-3-phenylthio-propionate ${ }^{6}$ (the precursor of ketene 20); imines $2 \mathbf{a}-\mathbf{c},{ }^{7} 5 \mathbf{a}-\mathbf{f},{ }^{7} \mathbf{8 a}-\mathbf{c},{ }^{8} 8 \mathbf{d}^{9}$ and $\mathbf{1 4 a}-\mathbf{f}^{7}$ were prepared according to published procedures. Toluene and xylenes were refluxed with sodium and freshly distilled prior to use. All reactions were performed under a nitrogen atmosphere. ${ }^{1} \mathrm{H}$ and ${ }^{13} \mathrm{C}$ NMR spectra were recorded in $\mathrm{CDCl}_{3}$ with TMS as an internal standard.

\section{The Determination of the cis / trans Ratios of the Crude $\beta$-Lactam Products.}

1. For the reactions of diazoacetate $\mathbf{1}$ and imines $\mathbf{5}$, the cis / trans ratios were obtained by the integral of the corresponding $\mathrm{C}(3)$ and $\mathrm{C}(4)$ protons of $\beta$-lactams in ${ }^{1} \mathrm{H}$ NMR spectra (protons of cis-isomers appear at about 4.7 and $4.9 \mathrm{ppm}$, while protons of trans-isomers appear at about 4.0 and $4.3 \mathrm{ppm}$ ) of the crude reaction mixture. See representative spectrum 1 for example.

2. For the reactions of diazoketones $\mathbf{1 0}$ and imine $\mathbf{5 f}$, the cis / trans ratios at $140{ }^{\circ} \mathrm{C}$ were obtained by the integral of the corresponding protons $\mathrm{C}(3)$ and $\mathrm{C}(4)$ protons of $\beta$-lactams in ${ }^{1} \mathrm{H}$ NMR spectra (protons of cis-isomers appear at about 4.8 and $5.1 \mathrm{ppm}$, while protons of trans-isomers appear at about 4.0 and 4.5 ppm) of the crude reaction mixture. See representative spectrum 2 for example. The cis / trans ratios at $130{ }^{\circ} \mathrm{C}$ were obtained by the HPLC analysis. See representative chromatogram 3 for example.

3. For the reactions of diazoacetate $\mathbf{1}$ and imines $\mathbf{1 4}$, the cis / trans ratios were obtained by the integral of the corresponding $\mathrm{C}(3)$ and $\mathrm{C}(4)$ protons of $\beta$-lactams in ${ }^{1} \mathrm{H}$ NMR spectra (protons of cis-isomers appear at about 5.0 and $5.5 \mathrm{ppm}$, while protons of trans-isomers appear at about 4.2 and $4.9 \mathrm{ppm}$ ) of the crude reaction mixture. See representative spectrum 5 for example.

The Reaction of Ketene 20 with imine 2c. The precursor of ketene 20, ethyl 2-diazo-3-oxo-3-phenylthiopropionate $(1.3 \mathrm{mmol})$, was dissolved in $15 \mathrm{~mL}$ of dry toluene and was heated to reflux for $2 \mathrm{~h}$. The resulting solution was cooled to $80^{\circ} \mathrm{C}$, and a solution of imine $2 \mathbf{c}(1 \mathrm{mmol})$ in $5 \mathrm{~mL}$ of toluene was added during 30 minutes. After the addition, the solution was maintained at $80{ }^{\circ} \mathrm{C}$ and stirred for $12 \mathrm{~h}$. The solvent was evaporated and the mixture was directly submitted to ${ }^{1} \mathrm{H}$ NMR analysis.

\footnotetext{
${ }^{4}$ Danheiser, R. L.; Okamoto, I.; Lawlor, M. D.; Lee, T. W. Org. Synth. Vol. 80, 160-171.

5 Bridson, J. N.; Hooz, J. Org. Synth. Coll. Vol. 6, 386.

${ }^{6}$ Padwa, A.; Sheehan, S. M.; Price, A. T. Tetrahedron Lett. 1994, 35, 849-852.

7 Linder, M. R.; Frey, W. U.; Podlech, J. J. Chem. Soc., Perkin Trans. 1 2001, 2566-2577.

${ }^{8}$ Wardrop, A. W. H.; Sainsbury, G. L.; Harrison, J. M.; Inch, T. D. J. Chem. Soc., Perkin Trans. 1 1976, 1279-1285.

${ }^{9}$ Nagarajan, K.; Venkateswarlu, A.; Kulkarni, C. L.; Shah, R. K. Indian J. Chem. 1974, 12, 227-235.
} 


\section{The Calculation of the cis / trans Ratios at $80^{\circ} \mathrm{C}$ Using the Eyring Equation}

The cis / trans ratios at 140 and $130^{\circ} \mathrm{C}$ are correlated with the Eyring Equation to give the $\Delta \Delta H^{\ddagger}$ and $\Delta \Delta S^{\neq}$data, and then the cis / trans ratios at $80^{\circ} \mathrm{C}$ were calculated (Table 1).

Table 1. The Calculation of the cis / trans Ratios at $80^{\circ} \mathrm{C}$

$$
\log \frac{\text { cis }}{\text { trans }}=\log \frac{k_{1}}{k_{2}}=-\frac{\Delta \Delta H^{\ddagger}}{2.303 R T}+\frac{\Delta \Delta S^{ \pm}}{2.303 R}
$$

\begin{tabular}{ccccccc}
\hline $\mathrm{R}$ & $p-\mathrm{MeO}$ & $p-\mathrm{Me}$ & $\mathrm{H}$ & $p-\mathrm{Cl}$ & $m-\mathrm{Cl}$ & $p-\mathrm{NO}_{2}$ \\
\hline cis / trans at $140^{\circ} \mathrm{C}$ & $1.95: 1$ & $1.23: 1$ & $1: 1.15$ & $1: 1.47$ & $1: 1.63$ & $1: 2.71$ \\
cis / trans at $130^{\circ} \mathrm{C}$ & $1.93: 1$ & $1.18: 1$ & $1: 1.26$ & $1: 1.67$ & $1: 1.84$ & $1: 3.25$ \\
\hline$\Delta \Delta H^{\mp} / \mathrm{kJ} \cdot \mathrm{mol}^{-1}$ & 1.1 & 5.7 & 13 & 18 & 17 & 25 \\
$\Delta \Delta S^{\neq} / \mathrm{J} \cdot \mathrm{K}^{-1} \cdot \mathrm{mol}^{-1}$ & 8.2 & 16 & 29 & 40 & 37 & 53 \\
\hline cis $/$ trans at $80^{\circ} \mathrm{C}$ & $1.85: 1$ & $1: 1.08$ & $1: 2.15$ & $1: 3.52$ & $1: 3.74$ & $1: 9.4$ \\
\hline
\end{tabular}

Meanwhile, $\Delta \Delta H^{\neq}$shows a good correlation with $\Delta \Delta S^{\neq}$(see figure below).

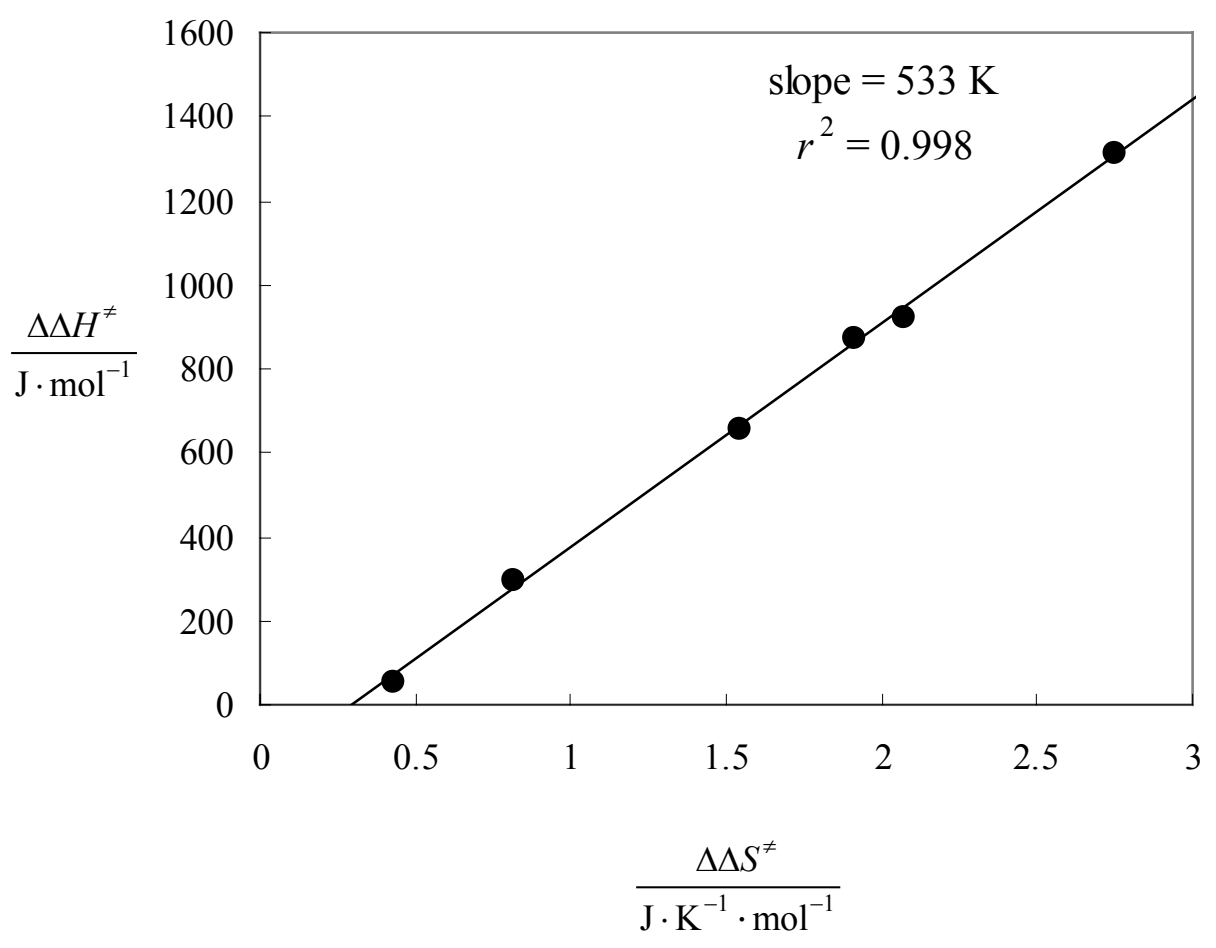




\section{Representative Spectra and Chromatogram}

1. Representative ${ }^{1} \mathrm{H}$ NMR Spectra of the Crude Products of the Reaction of $\mathbf{1}$ and $5 \mathrm{c}$
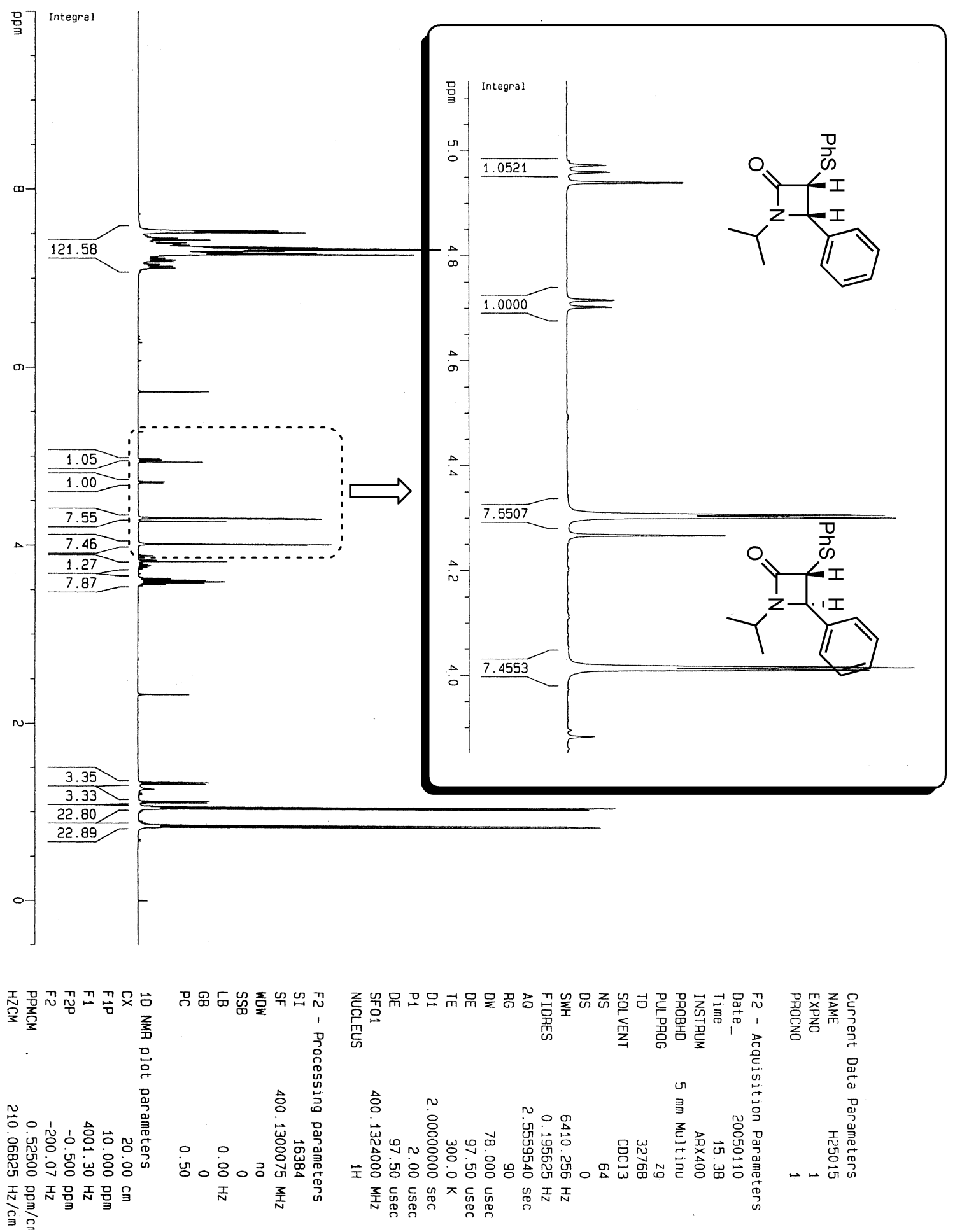
2. Representative ${ }^{1} \mathrm{H}$ NMR Spectra of the Crude Products of the Reaction of 10a and $5 f$

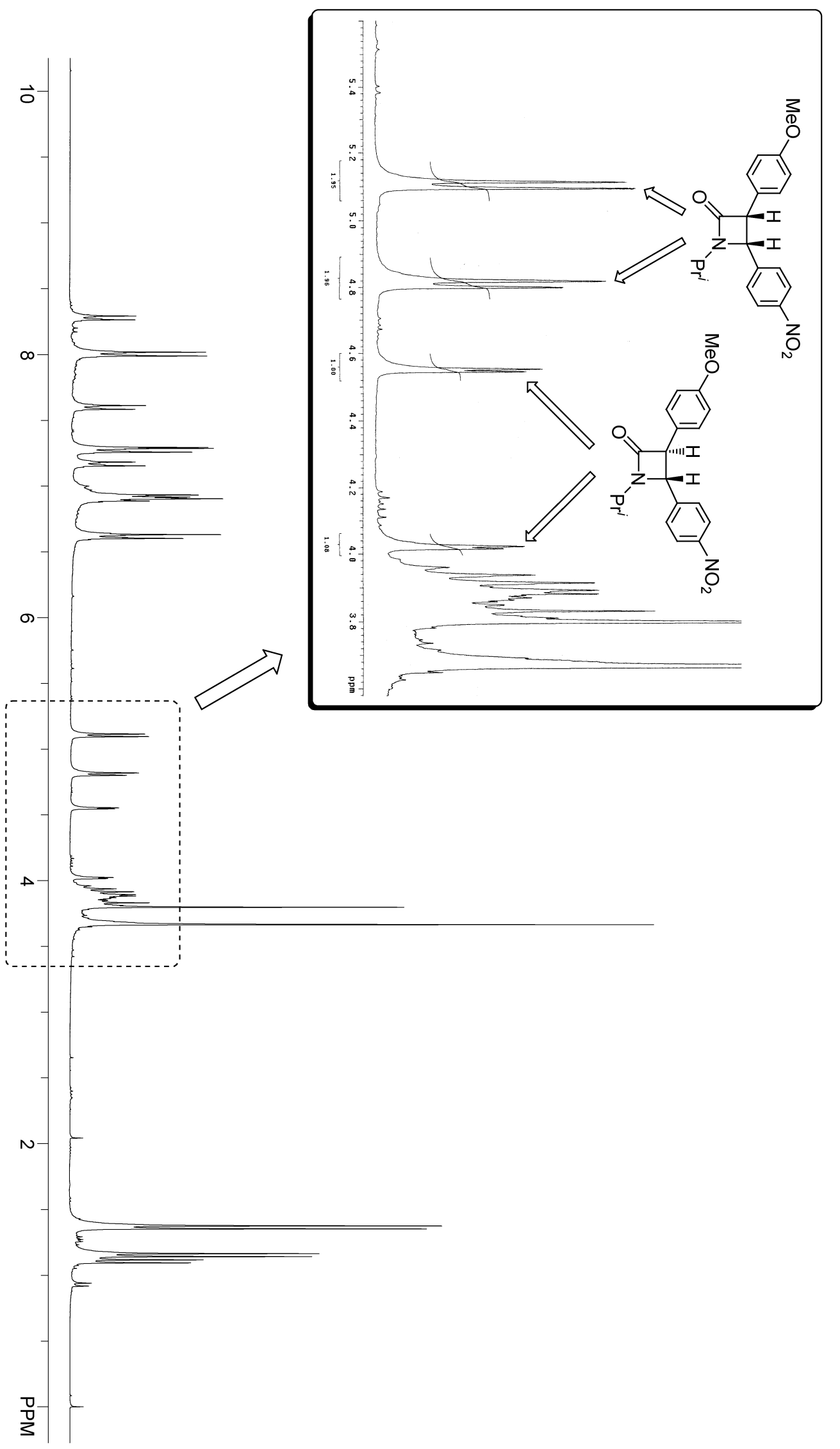


3. Representative HPLC Chromatogram of the Crude Products of the Reaction of 10a and 5f

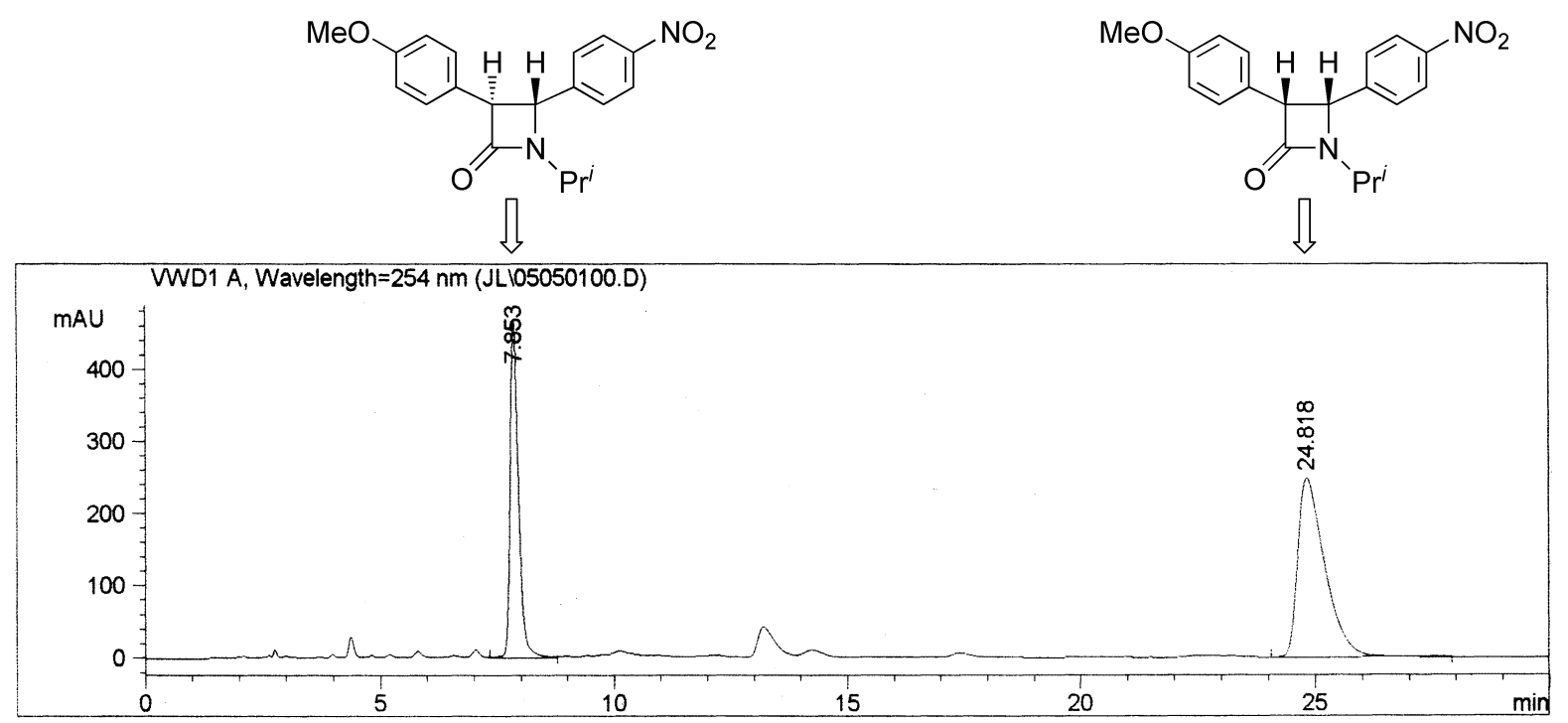

The cis / trans ratio of the $140{ }^{\circ} \mathrm{C}$ reactions were determined by ${ }^{1} \mathrm{H}$ NMR. Both the $140{ }^{\circ} \mathrm{C}$ and $130{ }^{\circ} \mathrm{C}$ reaction mixtures were analyzed by HPLC (Agilent ZORBAX SIL Column, elution with hexane $/ i$-PrOH 95:5 at $1.00 \mathrm{~mL} \cdot \mathrm{min}^{-1}$, detect at $254 \mathrm{~nm}$ ), and the response factors of the two diastereoisomers were calculated on the basis of the determined cis / trans ratio at $140{ }^{\circ} \mathrm{C}$ by ${ }^{1} \mathrm{H}$ NMR. Consequently the cis / trans ratio of the $130{ }^{\circ} \mathrm{C}$ reaction was calculated using the above response factors. 
4. Representative ${ }^{1} \mathrm{H}$ NMR Spectra of the Crude Product of the Reaction of 10f and 8d

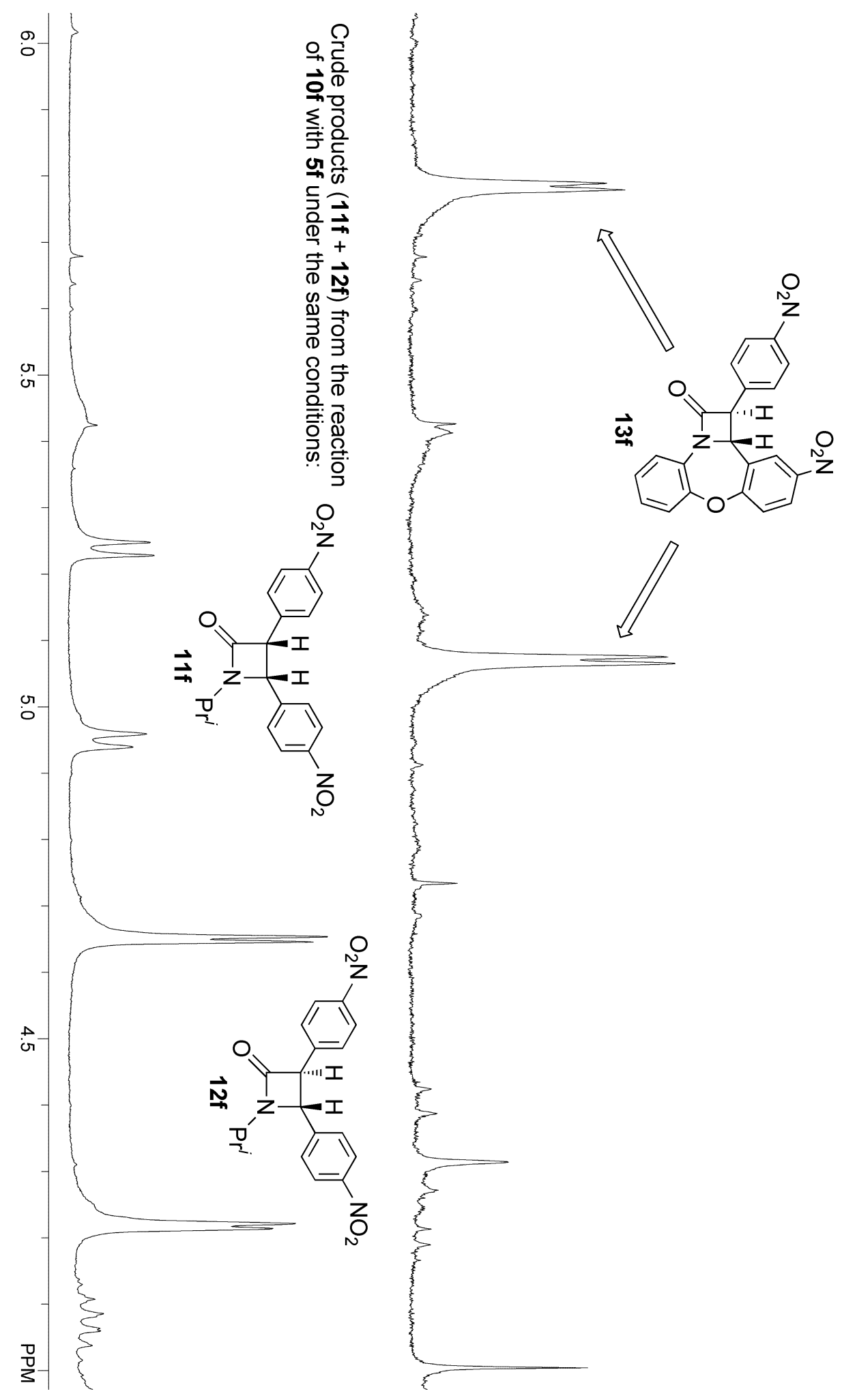


5. Representative ${ }^{1} \mathrm{H}$ NMR Spectra of the Crude Products of the Reaction of 1 and $14 a$

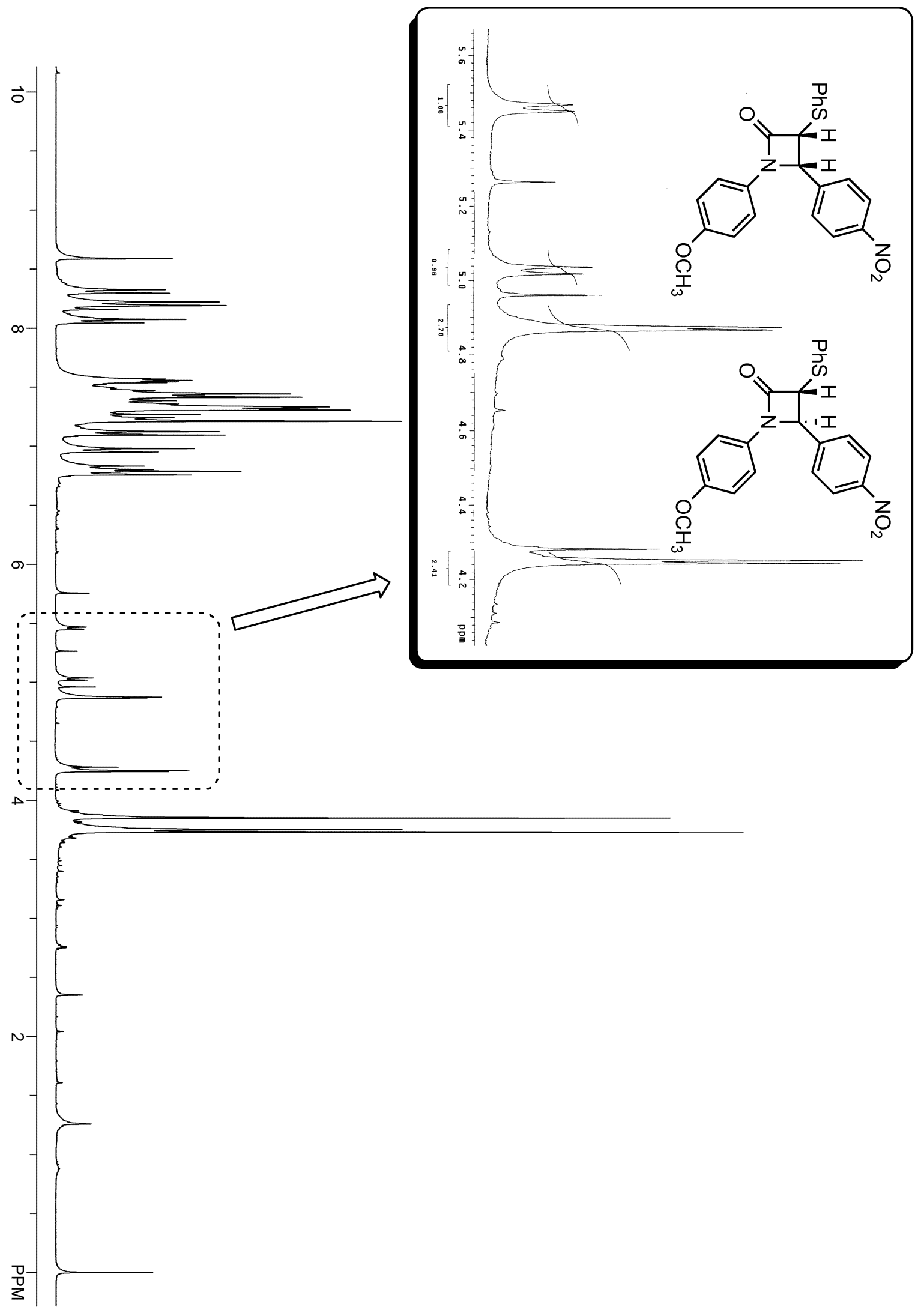




\section{The Spectroscopic Data for the Products}

( \pm )-trans-1-Benzyl-4-phenyl-3-phenylthioazetidin-2-one (4a)<smiles>O=C1[C@@H](c2ccccc2)[C@@H](c2ccccc2)N1Cc1ccccc1</smiles>

${ }^{1} \mathrm{H}$ NMR (300 MHz): $\delta 3.67(\mathrm{~d}, J=15.0 \mathrm{~Hz}, 1 \mathrm{H}), 4.15(\mathrm{dd}, J=0.5,2.1 \mathrm{~Hz}, 1 \mathrm{H}), 4.20(\mathrm{~d}, J=2.1 \mathrm{~Hz}, 1 \mathrm{H})$, $4.80(\mathrm{~d}, J=15.0 \mathrm{~Hz}, 1 \mathrm{H}), 6.72-6.76(\mathrm{~m}, 2 \mathrm{H}), 7.13-7.57(\mathrm{~m}, 13 \mathrm{H}) .{ }^{13} \mathrm{C} \mathrm{NMR}(75.5 \mathrm{MHz}): \delta 44.4,61.6$,

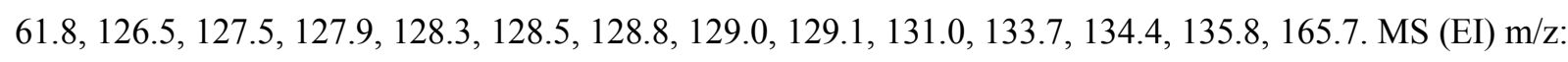
$345\left(\mathrm{M}^{+}, 0.5\right), 212(100), 91(61) . \mathrm{IR} v\left(\mathrm{~cm}^{-1}\right)$ : 1754. This compound has been reported in published literature ${ }^{10}$ however, no spectroscopic data was given.

( \pm )-cis-1-Isopropyl-4-phenyl-3-phenylthioazetidin-2-one (3b)

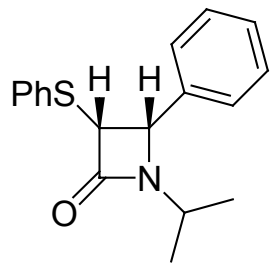

${ }^{1} \mathrm{H}$ NMR (300 MHz): $\delta 1.12(\mathrm{~d}, J=6.9 \mathrm{~Hz}, 3 \mathrm{H}), 1.33(\mathrm{~d}, J=6.9 \mathrm{~Hz}, 3 \mathrm{H}), 3.78(\mathrm{~m}, 1 \mathrm{H}), 4.71(\mathrm{~d}, J=5.4 \mathrm{~Hz}$, $1 \mathrm{H}), 4.97$ (d, $J=5.4 \mathrm{~Hz}, 1 \mathrm{H}), 7.13-7.38(\mathrm{~m}, 10 \mathrm{H}) .{ }^{13} \mathrm{C} \mathrm{NMR}(75.5 \mathrm{MHz}): \delta 20.2,21.1,45.7,58.2,59.1$, 126.7, 128.1, 128.65, 128.69, 130.5, 134.4, 135.2, 166.3. MS (ESI) m/z: $298(\mathrm{M}+\mathrm{H})^{+} . \mathrm{IR} v\left(\mathrm{~cm}^{-1}\right): 1749$.

( \pm )-trans-1-Isopropyl-4-phenyl-3-phenylthioazetidin-2-one (4b)

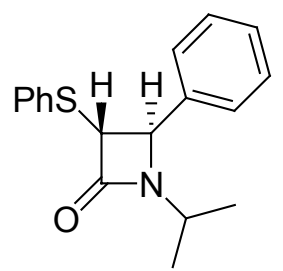

${ }^{1} \mathrm{H}$ NMR (300 MHz): $\delta 0.85(\mathrm{~d}, J=6.9 \mathrm{~Hz}, 3 \mathrm{H}), 1.06(\mathrm{~d}, J=6.9 \mathrm{~Hz}, 3 \mathrm{H}), 3.60(\mathrm{~m}, 1 \mathrm{H}), 4.02(\mathrm{~d}, J=2.1 \mathrm{~Hz}$, $1 \mathrm{H}), 4.32(\mathrm{~d}, J=2.1 \mathrm{~Hz}, 1 \mathrm{H}), 7.28-7.56(\mathrm{~m}, 10 \mathrm{H}) .{ }^{13} \mathrm{C}$ NMR $(75.5 \mathrm{MHz}): \delta 20.0,20.7,45.3,60.2,61.2$, 126.4, 128.0, 128.6, 128.7, 128.9, 131.3, 133.2, 137.6, 165.6. MS (ESI) m/z: $298(\mathrm{M}+\mathrm{H})^{+} . \mathrm{IR} v\left(\mathrm{~cm}^{-1}\right)$ : 1751.

${ }^{10}$ Sugano, Y.; Naruto, S. Chem. Lett., 1989, 1331-1334. 
( \pm )-cis-1-tert-Butyl-4-phenyl-3-phenylthioazetidin-2-one (3c)<smiles>O=C1[C@@H](c2ccccc2)[C@@H](c2ccccc2)N1Cc1ccccc1</smiles>

${ }^{1} \mathrm{H}$ NMR (300 MHz): $\delta 1.31(\mathrm{~s}, 9 \mathrm{H}), 4.66(\mathrm{~d}, J=5.4 \mathrm{~Hz}, 1 \mathrm{H}), 5.00(\mathrm{~d}, J=5.4 \mathrm{~Hz}, 1 \mathrm{H}), 7.15-7.36(\mathrm{~m}, 10 \mathrm{H})$. ${ }^{13} \mathrm{C}$ NMR (75.5 MHz): $\delta 28.1,54.9,57.5,59.3,126.7,128.0,128.6,128.7,130.6,134.6,136.4,166.5$. MS (EI) m/z: $311\left(\mathrm{M}^{+}, 0.8\right), 212(100), 57(13)$. IR $v\left(\mathrm{~cm}^{-1}\right): 1744$.

( \pm )-trans-1-Isopropyl-4-(4-methoxyphenyl)-3-phenylthioazetidin-2-one (7a)<smiles>COc1ccc([C@H]2[C@H](c3ccccc3)C(=O)N2C(C)C)cc1</smiles>

${ }^{1} \mathrm{H}$ NMR (300 MHz): $\delta 0.86(\mathrm{~d}, J=6.6 \mathrm{~Hz}, 3 \mathrm{H}), 1.05$ (d, $\left.J=6.6 \mathrm{~Hz}, 3 \mathrm{H}\right), 3.60$ (m, 1H), $3.80(\mathrm{~s}, 3 \mathrm{H}), 4.02$ $(\mathrm{d}, J=1.8 \mathrm{~Hz}, 1 \mathrm{H}), 4.28(\mathrm{~d}, J=1.8 \mathrm{~Hz}, 1 \mathrm{H}), 6.89-7.54(\mathrm{~m}, 9 \mathrm{H}) .{ }^{13} \mathrm{C} \mathrm{NMR}(75.5 \mathrm{MHz}): \delta 19.9,20.7,45.0$, 55.0, 60.0, 60.9, 114.1, 127.6, 127.8, 128.8, 129.3, 131.4, 132.9, 159.7, 165.5. MS (ESI) m/z: $328(\mathrm{M}+\mathrm{H})^{+}$. IR $v\left(\mathrm{~cm}^{-1}\right): 1748$.

( $($ )-cis-1-Isopropyl-4-(4-methylphenyl)-3-phenylthioazetidin-2-one (6b)<smiles>Cc1ccc([C@H]2[C@H](c3ccccc3)C(=O)N2C(C)C)cc1</smiles>

${ }^{1} \mathrm{H}$ NMR (300 MHz): $\delta 1.12(\mathrm{~d}, J=6.9 \mathrm{~Hz}, 3 \mathrm{H}), 1.33$ (d, $\left.J=6.9 \mathrm{~Hz}, 3 \mathrm{H}\right), 2.38(\mathrm{~s}, 3 \mathrm{H}), 3.78(\mathrm{~m}, 1 \mathrm{H}), 4.69$ (d, $J=5.1 \mathrm{~Hz}, 1 \mathrm{H}), 4.95(\mathrm{~d}, J=5.1 \mathrm{~Hz}, 1 \mathrm{H}), 7.15-7.33(\mathrm{~m}, 9 \mathrm{H}) . \mathrm{MS}(\mathrm{ESI}) \mathrm{m} / \mathrm{z}: 312(\mathrm{M}+\mathrm{H})^{+} . \mathrm{IR} v\left(\mathrm{~cm}^{-1}\right)$ : 1751.

( \pm --trans-1-Isopropyl-4-(4-methylphenyl)-3-phenylthioazetidin-2-one (7b)<smiles>Cc1ccc([C@H]2[C@H](c3ccccc3)C(=O)N2C(C)C)cc1</smiles> 
${ }^{1} \mathrm{H}$ NMR (300 MHz): $\delta 0.86(\mathrm{~d}, J=6.6 \mathrm{~Hz}, 3 \mathrm{H}), 1.05(\mathrm{~d}, J=6.6 \mathrm{~Hz}, 3 \mathrm{H}), 2.35(\mathrm{~s}, 3 \mathrm{H}), 3.60(\mathrm{~m}, 1 \mathrm{H}), 4.01$ (d, $J=2.1 \mathrm{~Hz}, 1 \mathrm{H}), 4.29$ (d, $J=2.1 \mathrm{~Hz}, 1 \mathrm{H}), 7.16-7.55$ (m, 9H). ${ }^{13} \mathrm{C}$ NMR $(75.5 \mathrm{MHz}): \delta 20.0,20.7,21.0$, 45.2, 60.1, 61.1, 126.4, 127.9, 128.8, 129.4, 131.4, 133.1, 134.6, 138.5, 165.6. MS (ESI) m/z: $312(\mathrm{M}+\mathrm{H})^{+}$. $\operatorname{IR} v\left(\mathrm{~cm}^{-1}\right): 1751$.

( \pm )-cis-4-(4-Chlorophenyl)-1-isopropyl-3-phenylthioazetidin-2-one (6d)

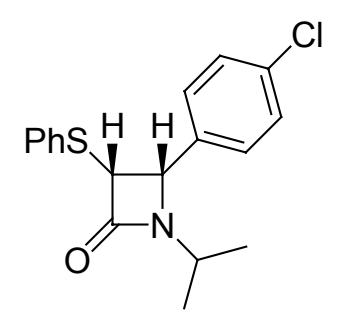

${ }^{1} \mathrm{H}$ NMR (300 MHz): $\delta 1.12(\mathrm{~d}, J=6.6 \mathrm{~Hz}, 3 \mathrm{H}), 1.32(\mathrm{~d}, J=6.6 \mathrm{~Hz}, 3 \mathrm{H}), 3.80(\mathrm{~m}, 1 \mathrm{H}), 4.73(\mathrm{~d}, J=5.4 \mathrm{~Hz}$, $1 \mathrm{H}), 4.95(\mathrm{~d}, J=5.4 \mathrm{~Hz}, 1 \mathrm{H}), 7.16-7.38(\mathrm{~m}, 9 \mathrm{H}) .{ }^{13} \mathrm{C}$ NMR $(75.5 \mathrm{MHz}): \delta 20.3,21.2,45.7,58.3,58.6$, $126.9,128.4,128.8,129.2,129.5,130.5,133.9,134.6,166.1$. MS (ESI) $\mathrm{m} / \mathrm{z}: 332(\mathrm{M}+\mathrm{H})^{+} . \mathrm{IR} v\left(\mathrm{~cm}^{-1}\right)$ : 1752.

( \pm )-trans-4-(4-Chlorophenyl)-1-isopropyl-3-phenylthioazetidin-2-one (7d)

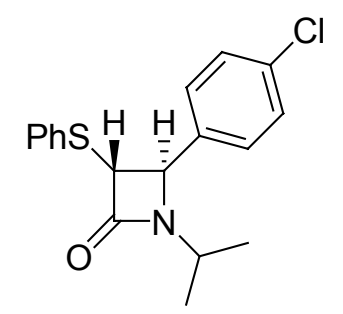

${ }^{1} \mathrm{H}$ NMR (300 MHz): $\delta 0.85$ (d, $\left.J=6.9 \mathrm{~Hz}, 3 \mathrm{H}\right), 1.04$ (d, $\left.J=6.9 \mathrm{~Hz}, 3 \mathrm{H}\right), 3.61(\mathrm{~m}, 1 \mathrm{H}), 3.99(\mathrm{~d}, J=2.1 \mathrm{~Hz}$, $1 \mathrm{H}), 4.29(\mathrm{~d}, J=2.1 \mathrm{~Hz}, 1 \mathrm{H}), 7.27-7.56(\mathrm{~m}, 9 \mathrm{H}) .{ }^{13} \mathrm{C}$ NMR $(75.5 \mathrm{MHz}): \delta 20.1,20.8,45.4,60.5,60.6$, $127.8,128.2,129.0,129.1,131.1,133.5,134.5,136.4,165.6 . \mathrm{MS}(\mathrm{ESI}) \mathrm{m} / \mathrm{z}: 332(\mathrm{M}+\mathrm{H})^{+} . \mathrm{IR} v\left(\mathrm{~cm}^{-1}\right)$ : 1751.

( \pm )-cis-4-(4-Trifluoromethylphenyl)-1-isopropyl-3-phenylthioazetidin-2-one (6e)

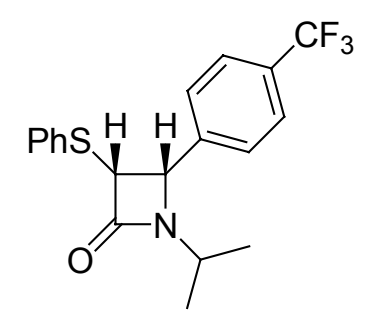

${ }^{1} \mathrm{H}$ NMR (300 MHz): $\delta 1.13(\mathrm{~d}, J=6.6 \mathrm{~Hz}, 3 \mathrm{H}), 1.33(\mathrm{~d}, J=6.6 \mathrm{~Hz}, 3 \mathrm{H}), 3.81(\mathrm{~m}, 1 \mathrm{H}), 4.79(\mathrm{~d}, J=5.1 \mathrm{~Hz}$, $1 \mathrm{H}), 5.02(\mathrm{~d}, J=5.1 \mathrm{~Hz}, 1 \mathrm{H}), 7.11-7.62(\mathrm{~m}, 9 \mathrm{H}) .{ }^{13} \mathrm{C}$ NMR $(75.5 \mathrm{MHz}): \delta 20.3,21.2,45.9,58.2,58.7$, $124.99,125.04,127.0,128.5,128.8,130.6,133.7,139.5,165.9$. MS (ESI) m/z: $366(\mathrm{M}+\mathrm{H})^{+}$. IR $v\left(\mathrm{~cm}^{-1}\right)$ : 
1753.

( \pm --trans $-4-(4-$ Trifluoromethylphenyl)-1-isopropyl-3-phenylthioazetidin-2-one (7e)<smiles>CC(C)N1C(=O)[C@]([PH3+])(c2ccccc2)[C@H]1c1ccc(C(F)(F)F)cc1</smiles>

${ }^{1} \mathrm{H}$ NMR (300 MHz): $\delta 0.85(\mathrm{~d}, J=6.9 \mathrm{~Hz}, 3 \mathrm{H}), 1.04(\mathrm{~d}, J=6.9 \mathrm{~Hz}, 3 \mathrm{H}), 3.63(\mathrm{~m}, 1 \mathrm{H}), 4.00(\mathrm{~d}, J=2.1 \mathrm{~Hz}$, $1 \mathrm{H}), 4.36(\mathrm{~d}, J=2.1 \mathrm{~Hz}, 1 \mathrm{H}), 7.31-7.66(\mathrm{~m}, 9 \mathrm{H}) .{ }^{13} \mathrm{C} \mathrm{NMR}(75.5 \mathrm{MHz}): \delta 20.1,20.9,45.7,60.7,60.9$, $125.94,125.99,126.8,128.5,129.2,130.8,133.9,142.2,165.7$. MS (ESI) m/z: $366(\mathrm{M}+\mathrm{H})^{+}$. IR $v\left(\mathrm{~cm}^{-1}\right)$ : 1756.

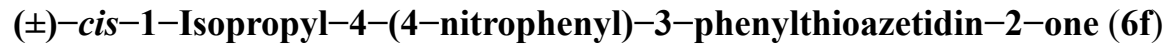<smiles>CC(C)N1C(=O)[C@]([PH3+])(c2ccc([N+](=O)[O-])cc2)[C@H]1c1ccccc1</smiles>

${ }^{1} \mathrm{H}$ NMR (300 MHz): $\delta 1.14$ (d, $\left.J=6.6 \mathrm{~Hz}, 3 \mathrm{H}\right), 1.34(\mathrm{~d}, J=6.6 \mathrm{~Hz}, 3 \mathrm{H}), 3.84(\mathrm{~m}, 1 \mathrm{H}), 4.85$ (d, $J=5.4 \mathrm{~Hz}$, $1 \mathrm{H}), 5.11(\mathrm{~d}, J=5.4 \mathrm{~Hz}, 1 \mathrm{H}), 7.10-7.18(\mathrm{~m}, 5 \mathrm{H}), 7.47-7.51(\mathrm{~m}, 2 \mathrm{H}), 8.19-8.22(\mathrm{~m}, 2 \mathrm{H}) .{ }^{13} \mathrm{C}$ NMR $(75.5$ MHz): $\delta 20.3,21.2,46.0,58.2,58.4,123.1,127.1,128.8,128.9,130.3,133.3,142.9,147.9,165.6$. MS (ESI) $\mathrm{m} / \mathrm{z}: 343(\mathrm{M}+\mathrm{H})^{+}$. IR $v\left(\mathrm{~cm}^{-1}\right): 1753$.

( \pm -) trans $^{-1}$-Isopropyl-4-(4-nitrophenyl)-3-phenylthioazetidin-2-one (7f)<smiles>CC(C)N1C(=O)[C@H](c2ccccc2)[C@H]1c1ccc([N+](=O)[O-])cc1</smiles>

${ }^{1} \mathrm{H}$ NMR (300 MHz): $\delta 0.86(\mathrm{~d}, J=6.9 \mathrm{~Hz}, 3 \mathrm{H}), 1.04(\mathrm{~d}, J=6.9 \mathrm{~Hz}, 3 \mathrm{H}), 3.65(\mathrm{~m}, 1 \mathrm{H}), 4.02(\mathrm{~d}, J=2.1 \mathrm{~Hz}$, 1H), 4.42 (d, $J=2.1 \mathrm{~Hz}, 1 \mathrm{H}), 7.35-7.60(\mathrm{~m}, 7 \mathrm{H}), 8.24-8.27$ (m, 2H). ${ }^{13} \mathrm{C}$ NMR (75.5 MHz): $\delta 20.1,20.8$, $45.7,60.2,61.1,124.2,127.2,128.7,129.2,130.3,134.1,145.4,148.0,165.4$. MS (ESI) m/z: $343(\mathrm{M}+\mathrm{H})^{+}$. $\operatorname{IR} v\left(\mathrm{~cm}^{-1}\right): 1755$. 
( \pm - trans $-4-M e t h y l-2-$ phenylthio-azeto[ $[1,2-d]$ dibenzo $[b, f]$ oxazepin-1-one (9a)<smiles></smiles>

${ }^{1} \mathrm{H}$ NMR $(300 \mathrm{MHz}): \delta 2.30(\mathrm{~s}, 3 \mathrm{H}), 4.75(\mathrm{~d}, J=3.0 \mathrm{~Hz}, 1 \mathrm{H}), 5.46(\mathrm{~d}, J=3.0 \mathrm{~Hz}, 1 \mathrm{H}), 6.90-7.99(\mathrm{~m}, 12 \mathrm{H})$. ${ }^{13} \mathrm{C}$ NMR (75.5 MHz): $\delta$ 20.8, 56.1, 59.7, 120.0, 121.3, 121.5, 124.7, 125.1, 126.4, 128.7, 128.8, 129.4, 129.6, 130.7, 131.3, 133.8, 135.0, 144.1, 155.9, 161.9. MS (EI) m/z: $359\left(\mathrm{M}^{+}, 17\right)$. IR $v\left(\mathrm{~cm}^{-1}\right): 1756$.

$( \pm)-$ trans $^{-2}-$ Phenylthio-azeto[1,2-d]dibenzo[b,floxazepin-1-one $(9 \mathrm{~b})$<smiles>O=C1[C@H]([PbH]c2ccccc2)[C@@H]2c3ccccc3Oc3ccccc3N12</smiles>

${ }^{1} \mathrm{H}$ NMR (300 MHz): $\delta 4.76(\mathrm{~d}, J=2.7 \mathrm{~Hz}, 1 \mathrm{H}), 5.48(\mathrm{~d}, J=2.7 \mathrm{~Hz}, 1 \mathrm{H}), 6.95-7.99(\mathrm{~m}, 13 \mathrm{H}) .{ }^{13} \mathrm{C}$ NMR (75.5 MHz): $\delta 56.3,59.7,120.0,121.5,121.6,124.7,125.2,125.3,125.9,128.7,129.3,129.4,129.5,130.4$, 131.3, 133.7, 143.9, 158.1, 161.8. MS (EI) m/z: $345\left(\mathrm{M}^{+}, 22\right)$. IR $v\left(\mathrm{~cm}^{-1}\right): 1736$.

$( \pm)-$ trans $-4-$ Chloro-2-phenylthio-azeto[1,2-d]dibenzo[b,f]oxazepin-1-one $(9 \mathrm{c})$<smiles>C[C@@]12c3cc(Cl)ccc3Oc3ccccc3N1C(=O)[C@@H]2[PbH]c1ccccc1</smiles>

${ }^{1} \mathrm{H}$ NMR (300 MHz): $\delta 4.71(\mathrm{~d}, J=2.4 \mathrm{~Hz}, 1 \mathrm{H}), 5.42(\mathrm{~d}, J=2.4 \mathrm{~Hz}, 1 \mathrm{H}), 7.00-7.99(\mathrm{~m}, 12 \mathrm{H}) .{ }^{13} \mathrm{C}$ NMR (75.5 MHz): $\delta 56.6,59.3,120.2,121.5,123.1,124.9,125.5,126.2,129.0,129.4,129.5,130.2,130.5,131.0$, 134.0, 143.7, 156.5, 161.6. MS (EI) m/z: $379\left(\mathrm{M}^{+}, 28\right)$. IR $v\left(\mathrm{~cm}^{-1}\right): 1765$. 
$( \pm)-$ trans $^{-4}-$ Nitro-2-phenylthio-azeto $[1,2-d]$ dibenzo $[b, f]$ oxazepin-1-one $(9 \mathrm{~d})$

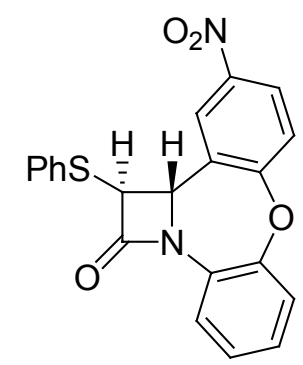

${ }^{1} \mathrm{H}$ NMR (300 MHz): $\delta 4.82(\mathrm{~d}, J=3.0 \mathrm{~Hz}, 1 \mathrm{H}), 5.42(\mathrm{~d}, J=3.0 \mathrm{~Hz}, 1 \mathrm{H}), 7.00-8.24(\mathrm{~m}, 12 \mathrm{H}) .{ }^{13} \mathrm{C}$ NMR (75.5 MHz): $\delta$ 57.4, 59.3, 120.3, 121.5, 122.1, 122.9, 125.2, 125.9, 126.0, 128.8, 129.2, 129.6, 130.5, 130.6, 134.1, 143.0, 144.3, 161.2, 162.3. MS (EI) m/z: $390\left(\mathrm{M}^{+}, 52\right)$. IR $v\left(\mathrm{~cm}^{-1}\right): 1753$.

( \pm )-cis-1-Isopropyl-3-(4-methoxyphenyl)-4-(4-nitrophenyl)azetidin-2-one (11a)<smiles>COc1ccc([C@H]2C(=O)N(C(C)C)[C@H]2c2ccc([N+](=O)[O-])cc2)cc1</smiles>

${ }^{1} \mathrm{H}$ NMR $(300 \mathrm{MHz}): \delta 1.15(\mathrm{~d}, J=6.6 \mathrm{~Hz}, 3 \mathrm{H}), 1.36(\mathrm{~d}, J=6.6 \mathrm{~Hz}, 3 \mathrm{H}), 3.67$ (s, 3H), $3.92(\mathrm{~m}, 1 \mathrm{H}), 4.81$ $(\mathrm{d}, J=5.7 \mathrm{~Hz}, 1 \mathrm{H}), 5.10(\mathrm{~d}, J=5.7 \mathrm{~Hz}, 1 \mathrm{H}), 6.60-8.02(\mathrm{~m}, 8 \mathrm{H}) .{ }^{13} \mathrm{C}$ NMR $(75.5 \mathrm{MHz}): \delta 20.3,21.3,45.5$, 55.0, 58.5, 59.6, 113.7, 123.1, 123.9, 128.2, 129.6, 144.5, 147.3, 158.5, 168.0. MS (ESI) m/z: $341(\mathrm{M}+\mathrm{H})^{+}$. IR $v\left(\mathrm{~cm}^{-1}\right): 1744$.

( \pm )-trans-1-Isopropyl-3-(4-methoxyphenyl)-4-(4-nitrophenyl)azetidin-2-one (12a)<smiles>COc1ccc([C@H]2C(=O)N(C(C)C)[C@H]2c2ccc([N+](=O)[O-])cc2)cc1</smiles>

${ }^{1} \mathrm{H}$ NMR (300 MHz): $\delta 1.11(\mathrm{~d}, J=6.6 \mathrm{~Hz}, 3 \mathrm{H}), 1.37(\mathrm{~d}, J=6.6 \mathrm{~Hz}, 3 \mathrm{H}), 3.81(\mathrm{~s}, 3 \mathrm{H}), 3.92(\mathrm{~m}, 1 \mathrm{H}), 4.02$ $(\mathrm{d}, J=2.1 \mathrm{~Hz}, 1 \mathrm{H}), 4.54(\mathrm{~d}, J=2.1 \mathrm{~Hz}, 1 \mathrm{H}), 6.89-8.30$ (m, 8H). ${ }^{13} \mathrm{C} \mathrm{NMR}(75.5 \mathrm{MHz}): \delta 20.7,21.3,45.5$, 55.3, 62.3, 64.1, 114.4, 124.3, 126.4, 127.2, 128.4, 146.8, 148.0, 159.3, 168.2. MS (ESI) m/z: $341(\mathrm{M}+\mathrm{H})^{+}$. $\operatorname{IR} v\left(\mathrm{~cm}^{-1}\right): 1747$. 
( $)^{-}-c i s-1-$ Isopropyl-3-(4-methylphenyl)-4-(4-nitrophenyl)azetidin-2-one (11b)<smiles>Cc1ccc([C@H]2C(=O)N(C(C)C)[C@H]2c2ccc([N+](=O)[O-])cc2)cc1</smiles>

${ }^{1} \mathrm{H}$ NMR (300 MHz): $\delta 1.15$ (d, $\left.J=6.6 \mathrm{~Hz}, 3 \mathrm{H}\right), 1.36$ (d, $\left.J=6.6 \mathrm{~Hz}, 3 \mathrm{H}\right), 2.16(\mathrm{~s}, 3 \mathrm{H}), 3.91(\mathrm{~m}, 1 \mathrm{H}), 4.82$ $(\mathrm{d}, J=5.7 \mathrm{~Hz}, 1 \mathrm{H}), 5.12(\mathrm{~d}, J=5.7 \mathrm{~Hz}, 1 \mathrm{H}), 6.88-8.00(\mathrm{~m}, 8 \mathrm{H}) .{ }^{13} \mathrm{C} \mathrm{NMR}(75.5 \mathrm{MHz}): \delta 20.2,20.8,21.2$, $45.5,58.3,59.9,123.0,128.2,128.3,128.8,128.9,136.8,144.4,147.2,167.8$. MS (ESI) m/z: $325(\mathrm{M}+\mathrm{H})^{+}$. IR $v\left(\mathrm{~cm}^{-1}\right): 1745$.

( \pm )-trans -1 -Isopropyl-3-(4-methylphenyl)-4-(4-nitrophenyl)azetidin-2-one (12b)<smiles>Cc1ccc([C@H]2C(=O)N(C(C)C)[C@H]2c2ccc([N+](=O)[O-])cc2)cc1</smiles>

${ }^{1} \mathrm{H}$ NMR (300 MHz): $\delta 1.11(\mathrm{~d}, J=6.6 \mathrm{~Hz}, 3 \mathrm{H}), 1.36(\mathrm{~d}, J=6.6 \mathrm{~Hz}, 3 \mathrm{H}), 2.34(\mathrm{~s}, 3 \mathrm{H}), 3.91(\mathrm{~m}, 1 \mathrm{H}), 4.04$ $(\mathrm{d}, J=2.4 \mathrm{~Hz}, 1 \mathrm{H}), 4.57$ (d, $J=2.4 \mathrm{~Hz}, 1 \mathrm{H}), 7.12-8.30$ (m, 8H). ${ }^{13} \mathrm{C}$ NMR $(75.5 \mathrm{MHz}): \delta 20.6,21.0,21.2$, $45.5,61.9,64.3,124.2,127.1,127.2,129.6,131.3,137.7,146.8,147.9,168.0$. MS (ESI) m/z: $325(\mathrm{M}+\mathrm{H})^{+}$. $\operatorname{IR} v\left(\mathrm{~cm}^{-1}\right): 1749$.

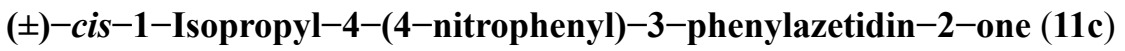<smiles>CC(C)N1C(=O)[C@H](c2ccccc2)[C@H]1c1ccc([N+](=O)[O-])cc1</smiles>

${ }^{1} \mathrm{H}$ NMR (300 MHz): $\delta 1.16(\mathrm{~d}, J=6.6 \mathrm{~Hz}, 3 \mathrm{H}), 1.37(\mathrm{~d}, J=6.6 \mathrm{~Hz}, 3 \mathrm{H}), 3.92(\mathrm{~m}, 1 \mathrm{H}), 4.87(\mathrm{~d}, J=5.7 \mathrm{~Hz}$, 1H), $5.14(\mathrm{~d}, J=5.7 \mathrm{~Hz}, 1 \mathrm{H}), 6.98-8.00(\mathrm{~m}, 9 \mathrm{H}) .{ }^{13} \mathrm{C} \mathrm{NMR}(75.5 \mathrm{MHz}): \delta 20.4,21.3,45.6,58.3,60.2$, 123.1, 127.3, 128.2, 128.3, 128.5, 131.9, 144.3, 147.3, 167.6. MS (ESI) $\mathrm{m} / \mathrm{z}: 311(\mathrm{M}+\mathrm{H})^{+} . \mathrm{IR} v\left(\mathrm{~cm}^{-1}\right)$ : 1744. 
( \pm )-trans-1-Isopropyl-4-(4-nitrophenyl)-3-phenylazetidin-2-one (12c)<smiles>CC(C)N1C(=O)[C@H](c2ccccc2)[C@H]1c1ccc([N+](=O)[O-])cc1</smiles>

${ }^{1} \mathrm{H}$ NMR (300 MHz): $\delta 1.12(\mathrm{~d}, J=6.6 \mathrm{~Hz}, 3 \mathrm{H}), 1.37(\mathrm{~d}, J=6.6 \mathrm{~Hz}, 3 \mathrm{H}), 3.92(\mathrm{~m}, 1 \mathrm{H}), 4.08(\mathrm{~d}, J=2.4 \mathrm{~Hz}$, $1 \mathrm{H}), 4.61(\mathrm{~d}, J=2.4 \mathrm{~Hz}, 1 \mathrm{H}), 7.24-8.31(\mathrm{~m}, 9 \mathrm{H}) .{ }^{13} \mathrm{C} \mathrm{NMR}(75.5 \mathrm{MHz}): \delta 20.7,21.3,45.5,61.8,64.5$, 124.3, 127.2, 127.9, 129.0, 134.3, 146.7, 148.0, 167.7. MS (ESI) m/z: $311(\mathrm{M}+\mathrm{H})^{+} . \mathrm{IR} v\left(\mathrm{~cm}^{-1}\right): 1749$.

( \pm )-cis-3-(4-Chlorophenyl)-1-isopropyl-4-(4-nitrophenyl)azetidin-2-one (11d)<smiles>CC(C)N1C(=O)[C@H](c2ccc(Cl)cc2)[C@H]1c1ccc([N+](=O)[O-])cc1</smiles>

${ }^{1} \mathrm{H}$ NMR (300 MHz): $\delta 1.15$ (d, $\left.J=6.6 \mathrm{~Hz}, 3 \mathrm{H}\right), 1.37$ (d, $\left.J=6.6 \mathrm{~Hz}, 3 \mathrm{H}\right), 3.90(\mathrm{~m}, 1 \mathrm{H}), 4.83(\mathrm{~d}, J=5.7 \mathrm{~Hz}$, $1 \mathrm{H}), 5.15(\mathrm{~d}, J=5.7 \mathrm{~Hz}, 1 \mathrm{H}), 6.94-8.04(\mathrm{~m}, 8 \mathrm{H}) .{ }^{13} \mathrm{C} \mathrm{NMR}(75.5 \mathrm{MHz}): \delta 20.3,21.2,45.7,58.2,59.3$, $123.3,128.2,128.5,129.8,130.5,133.2,143.9,147.4,167.1$. MS (ESI) m/z: $345(\mathrm{M}+\mathrm{H})^{+}$. IR $v\left(\mathrm{~cm}^{-1}\right)$ : 1746.

( \pm )-trans $-3-(4-$ Chlorophenyl)-1-isopropyl-4-(4-nitrophenyl)azetidin-2-one (12d)<smiles>CC(C)N1C(=O)[C@H](c2ccc(Cl)cc2)[C@@H]1c1ccc([N+](=O)[O-])cc1</smiles>

${ }^{1} \mathrm{H}$ NMR (300 MHz): $\delta 1.12(\mathrm{~d}, J=6.6 \mathrm{~Hz}, 3 \mathrm{H}), 1.37(\mathrm{~d}, J=6.6 \mathrm{~Hz}, 3 \mathrm{H}), 3.90(\mathrm{~m}, 1 \mathrm{H}), 4.07$ (d, $J=2.1 \mathrm{~Hz}$, $1 \mathrm{H}), 4.60(\mathrm{~d}, J=2.1 \mathrm{~Hz}, 1 \mathrm{H}), 7.20-8.30(\mathrm{~m}, 8 \mathrm{H}) .{ }^{13} \mathrm{C} \mathrm{NMR}(75.5 \mathrm{MHz}): \delta 20.5,21.1,45.5,61.6,63.6$, $124.2,127.2,128.5,129.0,132.8,133.6,146.3,147.9,167.1$. MS (ESI) m/z: $345(\mathrm{M}+\mathrm{H})^{+}$. IR $v\left(\mathrm{~cm}^{-1}\right)$ : 1751. 
( \pm )-cis-3-(3-Chlorophenyl)-1-isopropyl-4-(4-nitrophenyl)azetidin-2-one (11e)<smiles>CC(C)N1C(=O)[C@H](c2cccc(Cl)c2)[C@@H]1c1ccc([N+](=O)[O-])cc1</smiles>

${ }^{1} \mathrm{H}$ NMR (300 MHz): $\delta 1.16(\mathrm{~d}, J=6.6 \mathrm{~Hz}, 3 \mathrm{H}), 1.38(\mathrm{~d}, J=6.6 \mathrm{~Hz}, 3 \mathrm{H}), 3.91(\mathrm{~m}, 1 \mathrm{H}), 4.83(\mathrm{~d}, J=5.7 \mathrm{~Hz}$, $1 \mathrm{H}), 5.16(\mathrm{~d}, J=5.7 \mathrm{~Hz}, 1 \mathrm{H}), 7.01-8.05(\mathrm{~m}, 8 \mathrm{H}) .{ }^{13} \mathrm{C}$ NMR $(75.5 \mathrm{MHz}): \delta 20.3,21.2,45.7,58.2,59.4$, 123.2, 126.6, 127.5, 128.2, 128.5, 129.5, 134.0, 134.1, 143.8, 147.4, 166.8. MS (ESI) m/z: $345(\mathrm{M}+\mathrm{H})^{+}$. IR $v\left(\mathrm{~cm}^{-1}\right): 1746$.

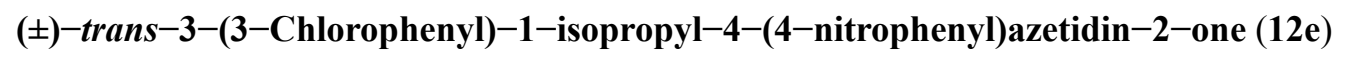<smiles>CC(C)N1C(=O)[C@H](c2cccc(Cl)c2)[C@@H]1c1ccc([N+](=O)[O-])cc1</smiles>

${ }^{1} \mathrm{H}$ NMR (300 MHz): $\delta 1.13(\mathrm{~d}, J=6.6 \mathrm{~Hz}, 3 \mathrm{H}), 1.38(\mathrm{~d}, J=6.6 \mathrm{~Hz}, 3 \mathrm{H}), 3.90(\mathrm{~m}, 1 \mathrm{H}), 4.08(\mathrm{~d}, J=2.1 \mathrm{~Hz}$, $1 \mathrm{H}), 4.62(\mathrm{~d}, J=2.1 \mathrm{~Hz}, 1 \mathrm{H}), 7.15-8.32(\mathrm{~m}, 8 \mathrm{H}) .{ }^{13} \mathrm{C} \mathrm{NMR}(75.5 \mathrm{MHz}): \delta 20.6,21.2,45.6,61.4,63.7$, 124.3, 125.4, 127.2, 127.3, 128.0, 130.2, 134.7, 136.2, 146.2, 148.0, 166.8. MS (ESI) m/z: $345(\mathrm{M}+\mathrm{H})^{+}$. IR $v\left(\mathrm{~cm}^{-1}\right): 1750$.

( \pm )-cis-1-Isopropyl-3,4-bis(4-nitrophenyl)azetidin-2-one (11f)<smiles>CC(C)N1C(=O)[C@H](c2ccc([N+](=O)[O-])cc2)[C@H]1c1ccc([N+](=O)[O-])cc1</smiles>

${ }^{1} \mathrm{H}$ NMR (300 MHz): $\delta 1.18(\mathrm{~d}, J=6.6 \mathrm{~Hz}, 3 \mathrm{H}), 1.40(\mathrm{~d}, J=6.6 \mathrm{~Hz}, 3 \mathrm{H}), 3.92(\mathrm{~m}, 1 \mathrm{H}), 4.97(\mathrm{~d}, J=5.7 \mathrm{~Hz}$, $1 \mathrm{H}), 5.27(\mathrm{~d}, J=5.7 \mathrm{~Hz}, 1 \mathrm{H}), 7.22-8.05(\mathrm{~m}, 8 \mathrm{H}) .{ }^{13} \mathrm{C} \mathrm{NMR}(75.5 \mathrm{MHz}): \delta 20.3,21.1,46.0,58.1,59.2$, 123.5, 128.1, 129.4, 130.0, 139.7, 143.3, 146.8, 147.6, 166.2. MS (ESI) m/z: $356(\mathrm{M}+\mathrm{H})^{+}$. IR $v\left(\mathrm{~cm}^{-1}\right)$ : 1748. 
( \pm )-trans $-1-$ Isopropyl-3,4-bis(4-nitrophenyl)azetidin-2-one (12f)<smiles>CC(C)N1C(=O)[C@H](c2ccc([N+](=O)[O-])cc2)[C@H]1c1ccc([N+](=O)[O-])cc1</smiles>

${ }^{1} \mathrm{H}$ NMR (300 MHz): $\delta 1.14(\mathrm{~d}, J=6.9 \mathrm{~Hz}, 3 \mathrm{H}), 1.38(\mathrm{~d}, J=6.9 \mathrm{~Hz}, 3 \mathrm{H}), 3.90(\mathrm{~m}, 1 \mathrm{H}), 4.22(\mathrm{~d}, J=2.1 \mathrm{~Hz}$, $1 \mathrm{H}), 4.65(\mathrm{~d}, J=2.1 \mathrm{~Hz}, 1 \mathrm{H}), 7.45-8.34(\mathrm{~m}, 8 \mathrm{H}) .{ }^{13} \mathrm{C} \mathrm{NMR}(75.5 \mathrm{MHz}): \delta 20.6,21.3,45.9,61.2,63.7$, 124.3, 124.5, 127.3, 128.2, 141.6, 145.8, 147.5, 148.3, 166.0. MS (ESI) m/z: $356(\mathrm{M}+\mathrm{H})^{+}$. IR $v\left(\mathrm{~cm}^{-1}\right)$ : 1751.

( \pm -trans-2-(4-Methoxyphenyl)-4-Nitro-azeto[1,2-d]dibenzo[b,floxazepin-1-one (13a)<smiles>COc1ccc([C@H]2C(=O)N3c4ccccc4Oc4ccc([N+](=O)[O-])cc4[C@H]23)cc1</smiles>

${ }^{1} \mathrm{H}$ NMR (300 MHz): $\delta 3.83$ (s, 3H), $4.85(\mathrm{~d}, J=3.0 \mathrm{~Hz}, 1 \mathrm{H}), 5.60(\mathrm{~d}, J=3.0 \mathrm{~Hz}, 1 \mathrm{H}), 6.96-8.28(\mathrm{~m}, 11 \mathrm{H})$. ${ }^{13} \mathrm{C}$ NMR $(75.5 \mathrm{MHz}): \delta 55.3,58.7,59.8,114.6,120.3,121.5,122.2,122.9,124.8,125.1,125.9,126.0$, 128.7, 129.3, 131.8, 143.0, 144.4, 159.6, 162.7, 163.6. MS (EI) m/z: $388\left(\mathrm{M}^{+}, 15\right)$. IR $v\left(\mathrm{~cm}^{-1}\right): 1753$.

( $($ )-trans-2-(4-Methylphenyl)-4-Nitro-azeto[1,2-d] dibenzo $[b, f]$ oxazepin-1-one (13b)<smiles>Cc1ccc([C@H]2C(=O)N3c4ccccc4Oc4ccc([N+](=O)[O-])cc4[C@H]23)cc1</smiles>

${ }^{1} \mathrm{H}$ NMR (300 MHz): $\delta 2.38(\mathrm{~s}, 3 \mathrm{H}), 4.87(\mathrm{~d}, J=3.0 \mathrm{~Hz}, 1 \mathrm{H}), 5.63(\mathrm{~d}, J=3.0 \mathrm{~Hz}, 1 \mathrm{H}), 7.06-8.30(\mathrm{~m}, 11 \mathrm{H})$. ${ }^{13} \mathrm{C}$ NMR (75.5 MHz): $\delta$ 21.1, 59.1, 59.7, 120.3, 121.5, 122.2, 123.0, 124.8, 125.97, 126.00, 127.4, 129.4, 129.9, 130.1 131.9, 138.2, 143.0, 144.4, 162.7, 163.5. MS (EI) m/z: $372\left(\mathrm{M}^{+}, 53\right)$. IR $v\left(\mathrm{~cm}^{-1}\right): 1758$. 
( \pm -trans $-2-(4-C h l o r o p h e n y l)-4-N i t r o-a z e t o[1,2-d]$ dibenzo $[b, f]$ oxazepin-1-one (13d)<smiles>O=C1[C@H](c2ccc(Cl)cc2)[C@H]2c3cc([N+](=O)[O-])ccc3Oc3ccccc3N12</smiles>

${ }^{1} \mathrm{H}$ NMR (300 MHz): $\delta 4.90(\mathrm{~d}, J=3.0 \mathrm{~Hz}, 1 \mathrm{H}), 5.65(\mathrm{~d}, J=3.0 \mathrm{~Hz}, 1 \mathrm{H}), 7.07-8.32(\mathrm{~m}, 11 \mathrm{H}) .{ }^{13} \mathrm{C}$ NMR (75.5 MHz): $\delta 58.5,59.5,120.3,121.6,122.1,123.1,125.1,126.1,126.2,128.9,129.2,129.5,131.60$, 131.64, 134.4, 143.0, 144.5, 162.66, 162.72. MS (EI) m/z: $392\left(\mathrm{M}^{+}, 89\right) . \operatorname{IR~} v\left(\mathrm{~cm}^{-1}\right): 1747$.

( \pm --trans-4-Nitro-2-(4-Nitrophenyl)-azeto[1,2-d]dibenzo[b,floxazepin-1-one (13f)<smiles>O=C1[C@H](c2ccc([N+](=O)[O-])cc2)[C@@H]2c3cc([N+](=O)[O-])ccc3Oc3ccccc3N12</smiles>

${ }^{1} \mathrm{H}$ NMR (300 MHz): $\delta 5.04(\mathrm{~d}, J=3.0 \mathrm{~Hz}, 1 \mathrm{H}), 5.77(\mathrm{~d}, J=3.0 \mathrm{~Hz}, 1 \mathrm{H}), 7.12-8.36(\mathrm{~m}, 11 \mathrm{H}) . \mathrm{MS}(\mathrm{EI}) \mathrm{m} / \mathrm{z}$ : $403\left(\mathrm{M}^{+}, 32\right)$. IR $v\left(\mathrm{~cm}^{-1}\right): 1745$.

( \pm )-cis-1-(4-Methoxyphenyl)-4-(4-nitrophenyl)-3-phenylthioazetidin-2-one (15a)<smiles>COc1ccc(N2C(=O)[C@H](c3ccccc3)[C@H]2c2ccc([N+](=O)[O-])cc2)cc1</smiles>

${ }^{1} \mathrm{H}$ NMR (300 MHz): $\delta 3.75$ (s, 3H), $5.02(\mathrm{~d}, J=5.4 \mathrm{~Hz}, 1 \mathrm{H}), 5.46$ (d, $\left.J=5.4 \mathrm{~Hz}, 1 \mathrm{H}\right), 6.80-8.18(\mathrm{~m}, 13 \mathrm{H})$. ${ }^{13} \mathrm{C}$ NMR (75.5 MHz): $\delta 55.4,58.6,59.3,114.5,118.4,123.5,127.4,128.6,129.0,130.1,130.8,132.9$, 141.2, 148.0, 156.6, 162.4. MS (ESI) m/z: $407(\mathrm{M}+\mathrm{H})^{+}$. IR $v\left(\mathrm{~cm}^{-1}\right): 1754$. 
( \pm )-trans-1-(4-Methoxyphenyl)-4-(4-nitrophenyl)-3-phenylthioazetidin-2-one (16a)<smiles>COc1ccc(N2C(=O)[C@@H](c3ccccc3)[C@H]2c2ccc([N+](=O)[O-])cc2)cc1</smiles>

${ }^{1} \mathrm{H}$ NMR (300 MHz): $\delta 3.72(\mathrm{~s}, 3 \mathrm{H}), 4.26(\mathrm{~d}, J=2.4 \mathrm{~Hz}, 1 \mathrm{H}), 4.89(\mathrm{~d}, J=2.4 \mathrm{~Hz}, 1 \mathrm{H}), 6.75-8.21(\mathrm{~m}, 13 \mathrm{H})$. ${ }^{13} \mathrm{C}$ NMR (75.5 MHz): $\delta 55.3,61.86,61.94,114.4,118.4,124.4,126.8,128.5,129.3,129.9,131.3,132.8$, 143.6, 148.0, 156.5, 161.8. MS (ESI) m/z: $407(\mathrm{M}+\mathrm{H})^{+} . \mathrm{IR} v\left(\mathrm{~cm}^{-1}\right): 1753$.

( $($ )-cis-1-(4-Methylphenyl)-4-(4-nitrophenyl)-3-phenylthioazetidin-2-one (15b)<smiles>Cc1ccc(N2C(=O)[C@H](c3ccccc3)[C@H]2c2ccc([N+](=O)[O-])cc2)cc1</smiles>

${ }^{1} \mathrm{H}$ NMR (300 MHz): $\delta 2.28$ (s, 3H), $5.02(\mathrm{~d}, J=5.7 \mathrm{~Hz}, 1 \mathrm{H}), 5.47$ (d, $\left.J=5.7 \mathrm{~Hz}, 1 \mathrm{H}\right), 7.07-8.18(\mathrm{~m}, 13 \mathrm{H})$. ${ }^{13} \mathrm{C}$ NMR (75.5 MHz): $\delta 20.9,58.6,59.2,117.0,123.5,127.5,128.6,129.0,129.8,130.9,132.9,134.2$, 134.6, 141.2, 148.0, 162.7. MS (ESI) m/z: $391(\mathrm{M}+\mathrm{H})^{+} . \mathrm{IR} v\left(\mathrm{~cm}^{-1}\right): 1757$.

( \pm )-trans $-1-(4-$ Methylphenyl)-4-(4-nitrophenyl)-3-phenylthioazetidin-2-one (16b)<smiles>Cc1ccc(N2C(=O)[C@H](c3ccccc3)[C@H]2c2ccc([N+](=O)[O-])cc2)cc1</smiles>

${ }^{1} \mathrm{H}$ NMR (300 MHz): $\delta 2.25(\mathrm{~s}, 3 \mathrm{H}), 4.26(\mathrm{~d}, J=2.4 \mathrm{~Hz}, 1 \mathrm{H}), 4.91(\mathrm{~d}, J=2.4 \mathrm{~Hz}, 1 \mathrm{H}), 7.05-8.21(\mathrm{~m}, 13 \mathrm{H})$. ${ }^{13} \mathrm{C}$ NMR (75.5 MHz): $\delta$ 20.8, 61.76, 116.9, 124.4, 126.8, 128.5, 129.3, 129.7, 131.2, 132.8, 134.0, 134.4, 143.6, 148.0, 162.1. MS (ESI) m/z: $391(\mathrm{M}+\mathrm{H})^{+}$. IR $v\left(\mathrm{~cm}^{-1}\right): 1757$. 
$( \pm)-c i s-4-(4-N i t r o p h e n y l)-1-p h e n y l-3-$ phenylthioazetidin-2-one (15c)<smiles>O=C1[C@@H](c2ccccc2)[C@H](c2ccc([N+](=O)[O-])cc2)N1c1ccccc1</smiles>

${ }^{1} \mathrm{H}$ NMR (300 MHz): $\delta 5.04$ (d, $\left.J=5.7 \mathrm{~Hz}, 1 \mathrm{H}\right), 5.50(\mathrm{~d}, J=5.7 \mathrm{~Hz}, 1 \mathrm{H}), 7.12-8.20(\mathrm{~m}, 14 \mathrm{H}) .{ }^{13} \mathrm{C}$ NMR (75.5 MHz): $\delta 58.6,59.3$, 117.1, 123.6, 124.8, 127.6, 128.6, 129.0, 129.4, 131.0, 132.8, 136.6, 141.0, 148.1, 163.0. MS (ESI) m/z: $377(\mathrm{M}+\mathrm{H})^{+}$. IR $v\left(\mathrm{~cm}^{-1}\right): 1759$.

( \pm -trans $-4-(4-$ Nitrophenyl)-1-phenyl-3-phenylthioazetidin-2-one (16c)<smiles>O=C1[C@@H](c2ccccc2)[C@@H](c2ccc([N+](=O)[O-])cc2)N1c1ccccc1</smiles>

${ }^{1} \mathrm{H}$ NMR (300 MHz): $\delta 4.28(\mathrm{~d}, J=2.4 \mathrm{~Hz}, 1 \mathrm{H}), 4.93(\mathrm{~d}, J=2.4 \mathrm{~Hz}, 1 \mathrm{H}), 7.07-8.21(\mathrm{~m}, 14 \mathrm{H}) .{ }^{13} \mathrm{C} \mathrm{NMR}$ (75.5 MHz): $\delta 61.80,61.82,116.9,124.4,124.7,126.8,128.5,129.2,129.3,131.2,132.8,136.5,143.5$, 148.0, 162.4. MS (ESI) m/z: $377(\mathrm{M}+\mathrm{H})^{+}$. IR $v\left(\mathrm{~cm}^{-1}\right): 1759$.

( \pm )-cis-1-(4-Chlorophenyl)-4-(4-nitrophenyl)-3-phenylthioazetidin-2-one (15d)<smiles>O=C1[C@@H](c2ccccc2)[C@@H](c2ccc([N+](=O)[O-])cc2)N1c1ccc(Cl)cc1</smiles>

${ }^{1} \mathrm{H}$ NMR $(300 \mathrm{MHz}): \delta 5.06(\mathrm{~d}, J=5.4 \mathrm{~Hz}, 1 \mathrm{H}), 5.49(\mathrm{~d}, J=5.4 \mathrm{~Hz}, 1 \mathrm{H}), 7.21-8.19(\mathrm{~m}, 13 \mathrm{H}) .{ }^{13} \mathrm{C} \mathrm{NMR}$ (75.5 MHz): $\delta 58.8,59.4,118.3,123.6,127.6,128.5$, 129.0, 129.4, 129.9, 130.9, 132.6, 135.1, 140.5, 148.1, 163.0. MS (ESI) m/z: $411(\mathrm{M}+\mathrm{H})^{+}$. IR $v\left(\mathrm{~cm}^{-1}\right): 1761$. 
( \pm )-trans-1-(4-Chlorophenyl)-4-(4-nitrophenyl)-3-phenylthioazetidin-2-one (16d)<smiles>O=C1[C@@H](c2ccccc2)[C@@H](c2ccc([N+](=O)[O-])cc2)N1c1ccc(Cl)cc1</smiles>

${ }^{1} \mathrm{H}$ NMR (300 MHz): $\delta 4.28(\mathrm{~d}, J=2.4 \mathrm{~Hz}, 1 \mathrm{H}), 4.89$ (d, $\left.J=2.4 \mathrm{~Hz}, 1 \mathrm{H}\right), 7.10-8.24(\mathrm{~m}, 13 \mathrm{H}) .{ }^{13} \mathrm{C}$ NMR (75.5 MHz): $\delta$ 62.0, 62.2, 118.2, 124.6, 126.8, 128.8, 129.37, 129.42, 129.9, 130.9, 133.1, 135.0, 143.0, 148.2, 162.4. MS (ESI) m/z: $411(\mathrm{M}+\mathrm{H})^{+}$. IR $v\left(\mathrm{~cm}^{-1}\right): 1760$.

( \pm -cis-1-(4-Acetylphenyl)-4-(4-nitrophenyl)-3-phenylthioazetidin-2-one (15e)<smiles>O=C1[C@H](c2ccc([N+](=O)[O-])cc2)[C@@H](c2ccccc2)[C@@H]1c1ccc(Cl)cc1</smiles>

${ }^{1} \mathrm{H}$ NMR (300 MHz): $\delta 2.54$ (s, 3H), 5.09 (d, $\left.J=5.4 \mathrm{~Hz}, 1 \mathrm{H}\right), 5.57$ (d, $\left.J=5.4 \mathrm{~Hz}, 1 \mathrm{H}\right), 7.23-8.21(\mathrm{~m}, 13 \mathrm{H})$. ${ }^{13} \mathrm{C}$ NMR (75.5 MHz): $\delta$ 26.4, 59.0, 59.5, 116.8, 123.7, 127.8, 128.5, 129.1, 129.9, 131.1, 132.5, 133.3, 140.2, 140.4, 148.2, 163.4, 196.4. MS (ESI) m/z: $419(\mathrm{M}+\mathrm{H})^{+}$. IR $v\left(\mathrm{~cm}^{-1}\right): 1678,1764$.

( \pm -trans-1-(4-Acetylphenyl)-4-(4-nitrophenyl)-3-phenylthioazetidin-2-one (16e)<smiles>O=C1[C@@H](c2ccccc2)[C@@H](c2ccc([N+](=O)[O-])cc2)N1c1ccc(Cl)cc1</smiles>

${ }^{1} \mathrm{H}$ NMR (300 MHz): $\delta 2.52$ (s, 3H), $4.32(\mathrm{~d}, J=2.4 \mathrm{~Hz}, 1 \mathrm{H}), 4.98(\mathrm{~d}, J=2.4 \mathrm{~Hz}, 1 \mathrm{H}), 7.22-8.24(\mathrm{~m}, 13 \mathrm{H})$.

${ }^{13} \mathrm{C}$ NMR (75.5 MHz): $\delta$ 26.3, 62.0, 62.2, 116.6, 124.6, 126.7, 128.8, 129.4, 129.8, 130.7, 133.1, 133.2, 140.1, 142.9, 148.2, 162.8, 196.4. MS (ESI) m/z: $419(\mathrm{M}+\mathrm{H})^{+}$. IR $v\left(\mathrm{~cm}^{-1}\right): 1677,1763$. 
( \pm )-cis-1-Isopropyl-4-(4-methoxyphenyl)-3-phenoxyazetidin-2-one (17a)<smiles>COc1ccc([C@H]2[C@H](c3ccccc3)C(=O)N2C(C)C)cc1</smiles>

${ }^{1} \mathrm{H}$ NMR (300 MHz): $\delta 1.06(\mathrm{~d}, J=6.9 \mathrm{~Hz}, 3 \mathrm{H}), 1.29(\mathrm{~d}, J=6.9 \mathrm{~Hz}, 3 \mathrm{H}), 3.74(\mathrm{~s}, 3 \mathrm{H}), 3.85(\mathrm{~m}, 1 \mathrm{H}), 4.91$ $(\mathrm{d}, J=4.5 \mathrm{~Hz}, 1 \mathrm{H}), 5.32$ (d, $J=4.5 \mathrm{~Hz}, 1 \mathrm{H}), 6.72-7.33$ (m, 9H). ${ }^{13} \mathrm{C}$ NMR $(75.5 \mathrm{MHz}): \delta 20.0,21.2,44.8$, $55.0,60.5,80.8,113.4,115.4,121.7,126.1,129.0,129.9,156.9,159.6,165.3 . \mathrm{MS}(\mathrm{ESI}) \mathrm{m} / \mathrm{z}: 312(\mathrm{M}+\mathrm{H})^{+}$. IR $v\left(\mathrm{~cm}^{-1}\right): 1751$.

( \pm )-trans-1-Isopropyl-4-(4-methoxyphenyl)-3-phenoxyazetidin-2-one (17b)<smiles>COc1ccc([C@H]2[C@H]([C@H](O)c3ccccc3)C(=O)N2C(C)C)cc1</smiles>

${ }^{1} \mathrm{H}$ NMR (300 MHz): $\delta 1.06$ (d, $\left.J=6.9 \mathrm{~Hz}, 3 \mathrm{H}\right), 1.31(\mathrm{~d}, J=6.9 \mathrm{~Hz}, 3 \mathrm{H}), 3.77(\mathrm{~m}, 1 \mathrm{H}), 3.86(\mathrm{~s}, 3 \mathrm{H}), 4.50$ $(\mathrm{d}, J=1.2 \mathrm{~Hz}, 1 \mathrm{H}), 4.92$ (d, $J=1.2 \mathrm{~Hz}, 1 \mathrm{H}), 6.75-7.34$ (m, 9H). ${ }^{13} \mathrm{C}$ NMR $(75.5 \mathrm{MHz}): \delta 20.1,21.2,45.1$, $55.3,62.3,86.5,114.4,115.2,121.8,128.2,128.9,129.5,157.1,160.1,165.1$. MS (ESI) m/z: $312(\mathrm{M}+\mathrm{H})^{+}$. IR $v\left(\mathrm{~cm}^{-1}\right): 1759$.

( \pm )-cis-1-Isopropyl-4-(4-methoxyphenyl)-3-(1,3-dioxoisoindolin-2-yl)azetidin-2-one (18a)<smiles>COc1ccc(C2C(N3C(=O)c4ccccc4C3=O)C(=O)N2C(C)C)cc1</smiles>

${ }^{1} \mathrm{H}$ NMR (300 MHz): $\delta 1.26(\mathrm{~d}, J=6.6 \mathrm{~Hz}, 3 \mathrm{H}), 1.41(\mathrm{~d}, J=6.6 \mathrm{~Hz}, 3 \mathrm{H}), 3.67(\mathrm{~s}, 3 \mathrm{H}), 3.98(\mathrm{~m}, 1 \mathrm{H}), 5.04$ $(\mathrm{d}, J=5.1 \mathrm{~Hz}, 1 \mathrm{H}), 5.38$ (d, $J=5.1 \mathrm{~Hz}, 1 \mathrm{H}), 6.73-7.89$ (m, 8H). ${ }^{13} \mathrm{C}$ NMR $(75.5 \mathrm{MHz}): \delta 20.0,21.2,45.6$, $55.0,59.0,59.9,113.6,123.4,125.7,128.7,131.2,134.1,159.4,163.5,166.9 . \mathrm{MS}(\mathrm{ESI}) \mathrm{m} / \mathrm{z}: 365(\mathrm{M}+\mathrm{H})^{+}$. $\operatorname{IR} v\left(\mathrm{~cm}^{-1}\right): 1719,1755$. 
( \pm -trans-1-Isopropyl-4-(4-methoxyphenyl)-3-(1,3-dioxoisoindolin-2-yl)azetidin-2-one $(18 \mathrm{~b})$<smiles>COc1ccc(C2[C@H](N3C(=O)c4ccccc4C3=O)C(=O)N2C(C)C)cc1</smiles>

${ }^{1} \mathrm{H}$ NMR (300 MHz): $\delta 1.12(\mathrm{~d}, J=6.9 \mathrm{~Hz}, 3 \mathrm{H}), 1.41(\mathrm{~d}, J=6.9 \mathrm{~Hz}, 3 \mathrm{H}), 3.81(\mathrm{~s}, 3 \mathrm{H}), 3.85(\mathrm{~m}, 1 \mathrm{H}), 4.94$ $(\mathrm{d}, J=2.4 \mathrm{~Hz}, 1 \mathrm{H}), 5.11$ (d, $J=2.4 \mathrm{~Hz}, 1 \mathrm{H}), 6.91-7.85$ (m, 8H). ${ }^{13} \mathrm{C}$ NMR $(75.5 \mathrm{MHz}): \delta 19.9,20.6,45.2$, $55.1,58.7,61.7,114.2,123.4,127.9,128.9,131.5,134.2,159.9,164.6,166.8$. MS (ESI) m/z: $365(\mathrm{M}+\mathrm{H})^{+}$. $\operatorname{IR} v\left(\mathrm{~cm}^{-1}\right): 1717,1757$.

( \pm )-cis-1-Isopropyl-3-methyl-4-(4-nitrophenyl)azetidin-2-one (19a)<smiles>CC(C)N1C(=O)[C@H](C)[C@H]1c1ccc([N+](=O)[O-])cc1</smiles>

${ }^{1} \mathrm{H}$ NMR (300 MHz): $\delta 0.81(\mathrm{~d}, J=7.5 \mathrm{~Hz}, 3 \mathrm{H}), 1.16(\mathrm{~d}, J=6.6 \mathrm{~Hz}, 3 \mathrm{H}), 1.35(\mathrm{~d}, J=6.6 \mathrm{~Hz}, 3 \mathrm{H}), 3.53$ (m, $1 \mathrm{H}), 3.81(\mathrm{~m}, 1 \mathrm{H}), 4.90(\mathrm{~d}, J=5.7 \mathrm{~Hz}, 1 \mathrm{H}), 7.49-7.52(\mathrm{~m}, 2 \mathrm{H}), 8.27-8.30(\mathrm{~m}, 2 \mathrm{H}) .{ }^{13} \mathrm{C} \mathrm{NMR}(75.5 \mathrm{MHz})$ : $\delta$ 9.6, 20.3, 21.3, 45.4, 49.1, 56.8, 123.7, 128.1, 144.9, 147.7, 170.7. MS (ESI) m/z: $249(\mathrm{M}+\mathrm{H})^{+} . \mathrm{IR} v$ $\left(\mathrm{cm}^{-1}\right): 1746$.

( \pm )-trans-1-Isopropyl-3-methyl-4-(4-nitrophenyl)azetidin-2-one (19b)<smiles>CC(C)N1C(=O)[C@H](C)[C@H]1c1ccc([N+](=O)[O-])cc1</smiles>

${ }^{1} \mathrm{H}$ NMR (300 MHz): $\delta 1.06$ (d, $\left.J=6.6 \mathrm{~Hz}, 3 \mathrm{H}\right), 1.29(\mathrm{~d}, J=6.6 \mathrm{~Hz}, 3 \mathrm{H}), 1.40(\mathrm{~d}, J=7.5 \mathrm{~Hz}, 3 \mathrm{H}), 2.97$ (dq, $J=1.8,7.5 \mathrm{~Hz}, 1 \mathrm{H}), 3.83(\mathrm{~m}, 1 \mathrm{H}), 4.28(\mathrm{~d}, J=1.8 \mathrm{~Hz}, 1 \mathrm{H}), 7.59-7.62(\mathrm{~m}, 2 \mathrm{H}), 8.26-8.28(\mathrm{~m}, 2 \mathrm{H}) .{ }^{13} \mathrm{C}$ NMR (75.5 MHz): $\delta$ 12.7, 20.2, 21.0, 44.9, 54.6, 60.5, 123.9, 127.1, 147.2, 144.7, 170.3. MS (ESI) m/z: $249(\mathrm{M}+\mathrm{H})^{+}$. IR $v\left(\mathrm{~cm}^{-1}\right): 1747$. 
Ethyl ( \pm )-trans $-1-$ tert-butyl-4-phenyl-3-phenylthioazetidin-2-one-3-carboxylate (21b)

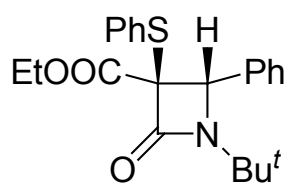

${ }^{1} \mathrm{H}$ NMR (300 MHz): $\delta 1.08$ (t, $\left.J=7.2 \mathrm{~Hz}, 3 \mathrm{H}\right), 1.33$ (s, 9H), $4.12(\mathrm{~m}, 2 \mathrm{H}), 5.23$ (s, 1H), 7.10-7.38 (m, $10 \mathrm{H}) .{ }^{13} \mathrm{C}$ NMR (75.5 MHz): $\delta$ 13.7, 28.0, 55.3, 62.4, 62.9, 69.4, 127.8, 128.0, 128.4, 128.7, 129.0, 130.6, 133.0 135.2, 163.2, 167.4. MS (ESI) m/z: $384(\mathrm{M}+\mathrm{H})^{+} . \mathrm{IR} v\left(\mathrm{~cm}^{-1}\right): 1720,1762$. 


\section{Copies of ${ }^{1} \mathrm{H}$ NMR Spectra of the Products}

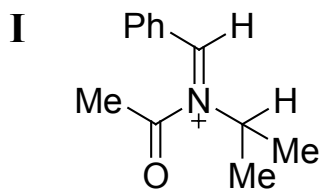

(containing some benzaldehyde and $N$-isopropyl acetamide)

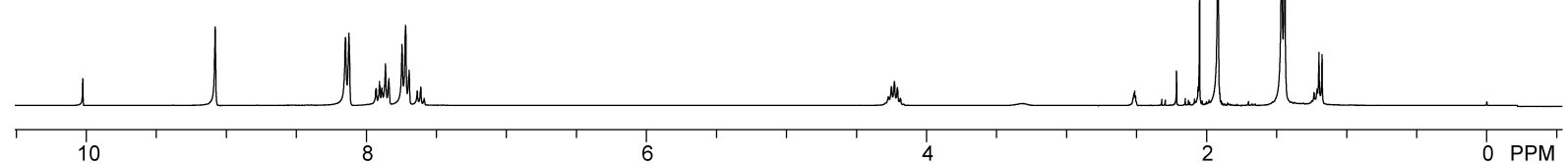

I NOE

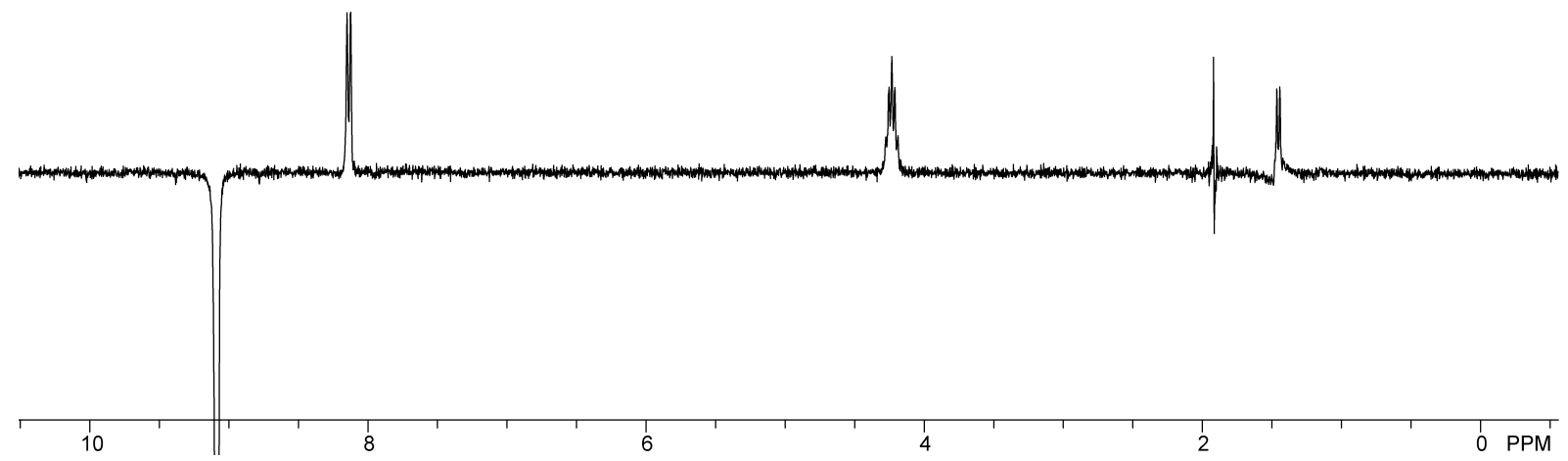




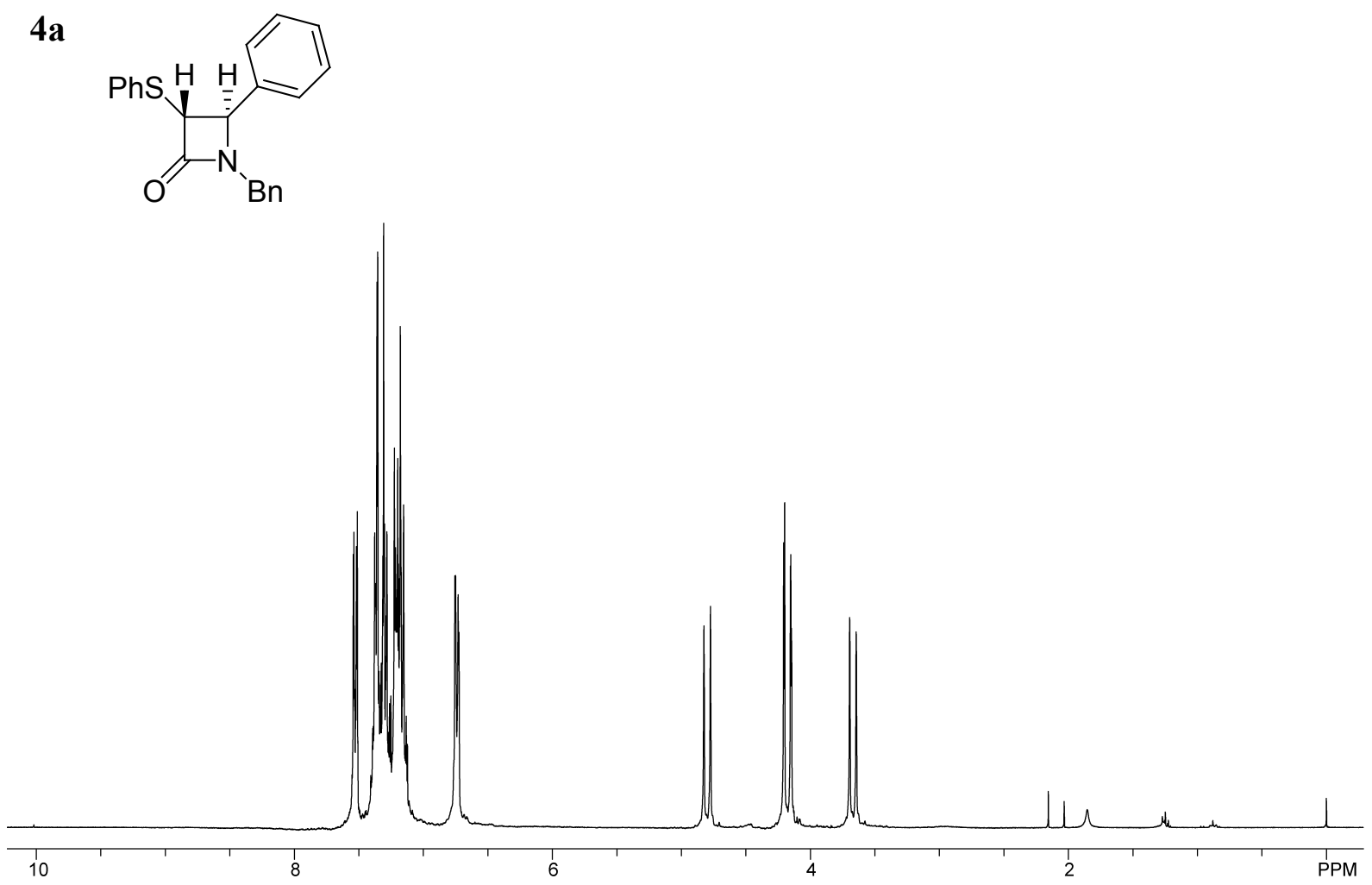


Supplemental Materials

S31

Bb
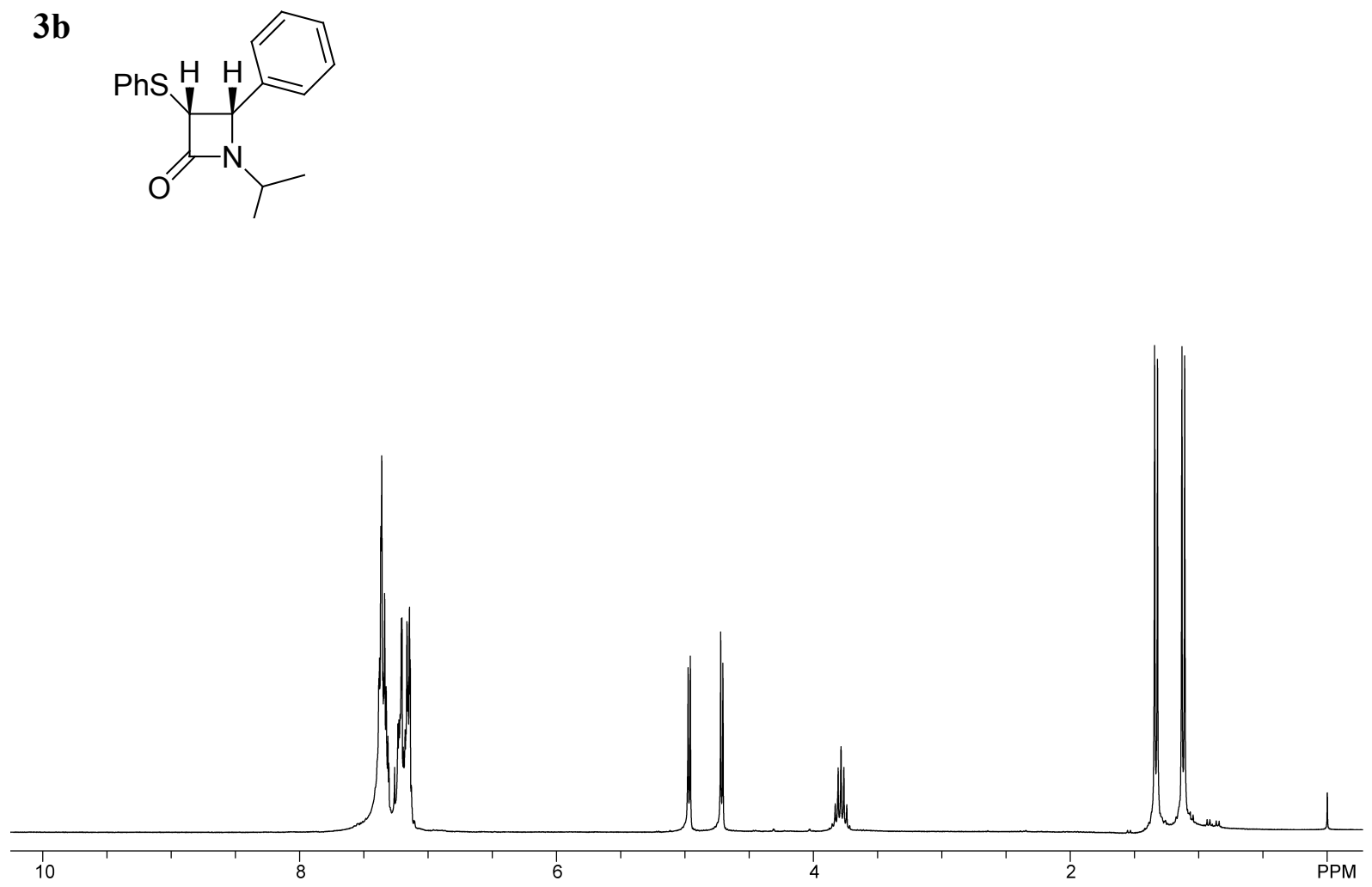

Ab
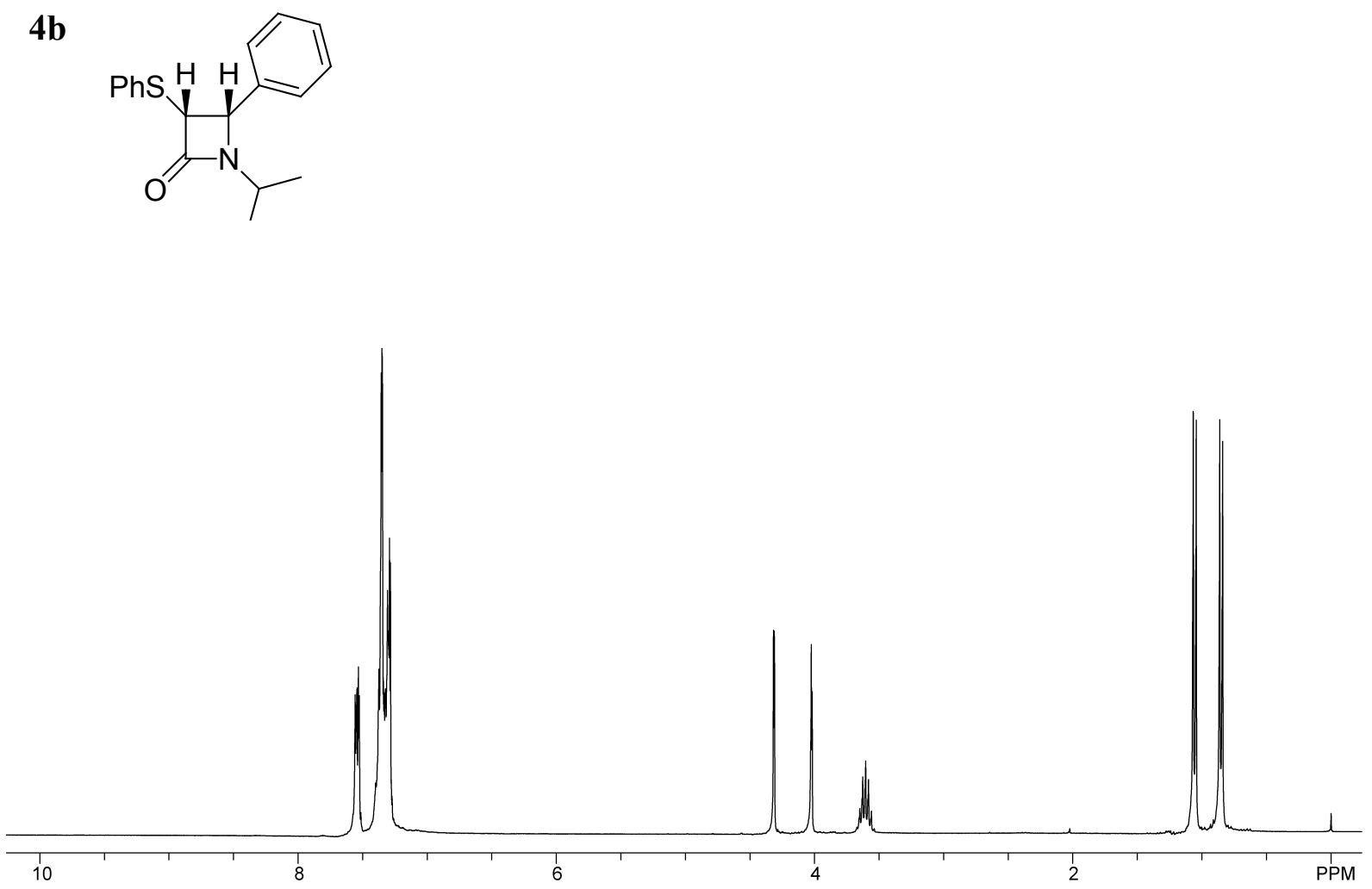
$3 c$<smiles>O=C1[C@@H]([PbH](c2ccccc2)c2ccccc2)[C@@H](c2ccccc2)N1Cc1ccccc1</smiles>

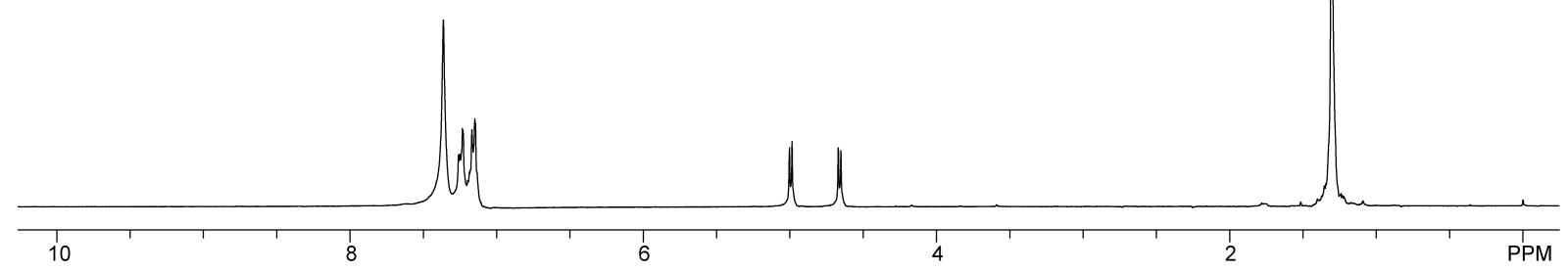


$7 \mathbf{a}$
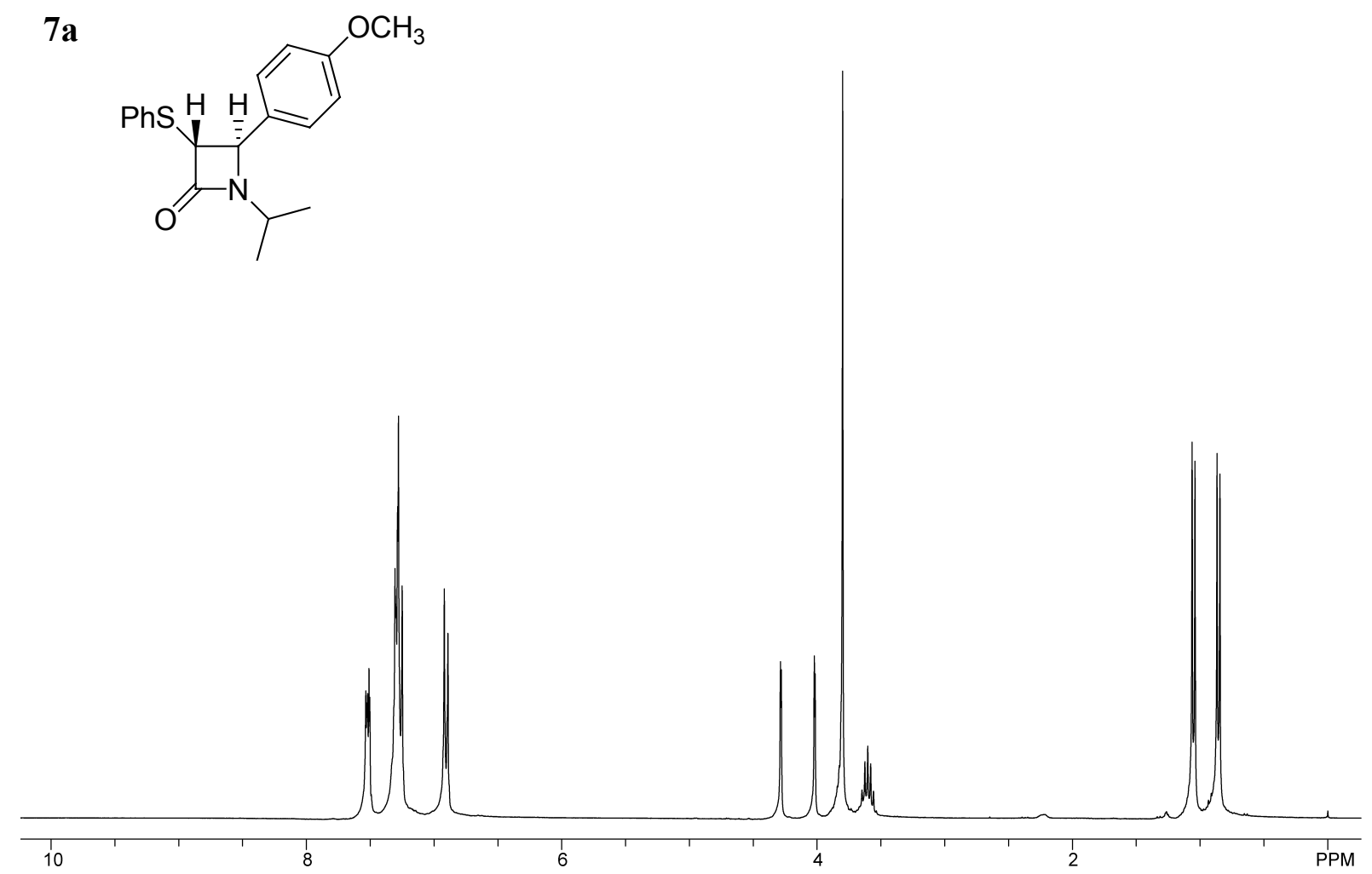
6b

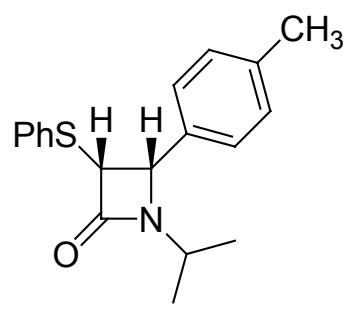

(containing some 7b)

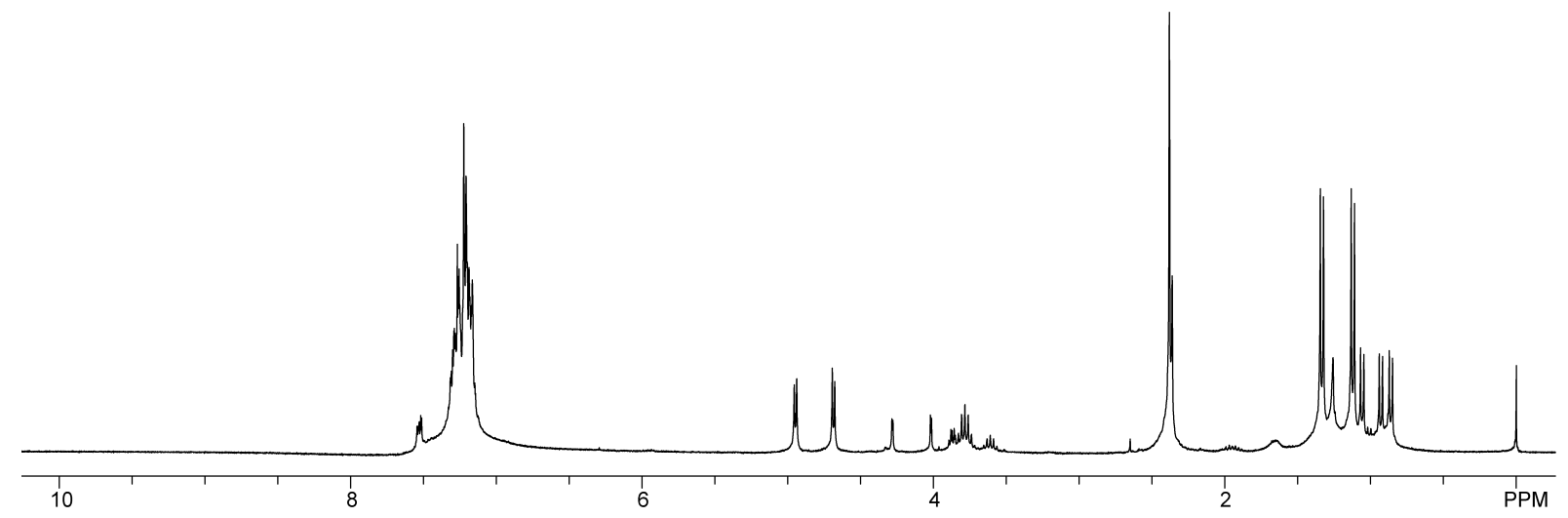

$7 b$
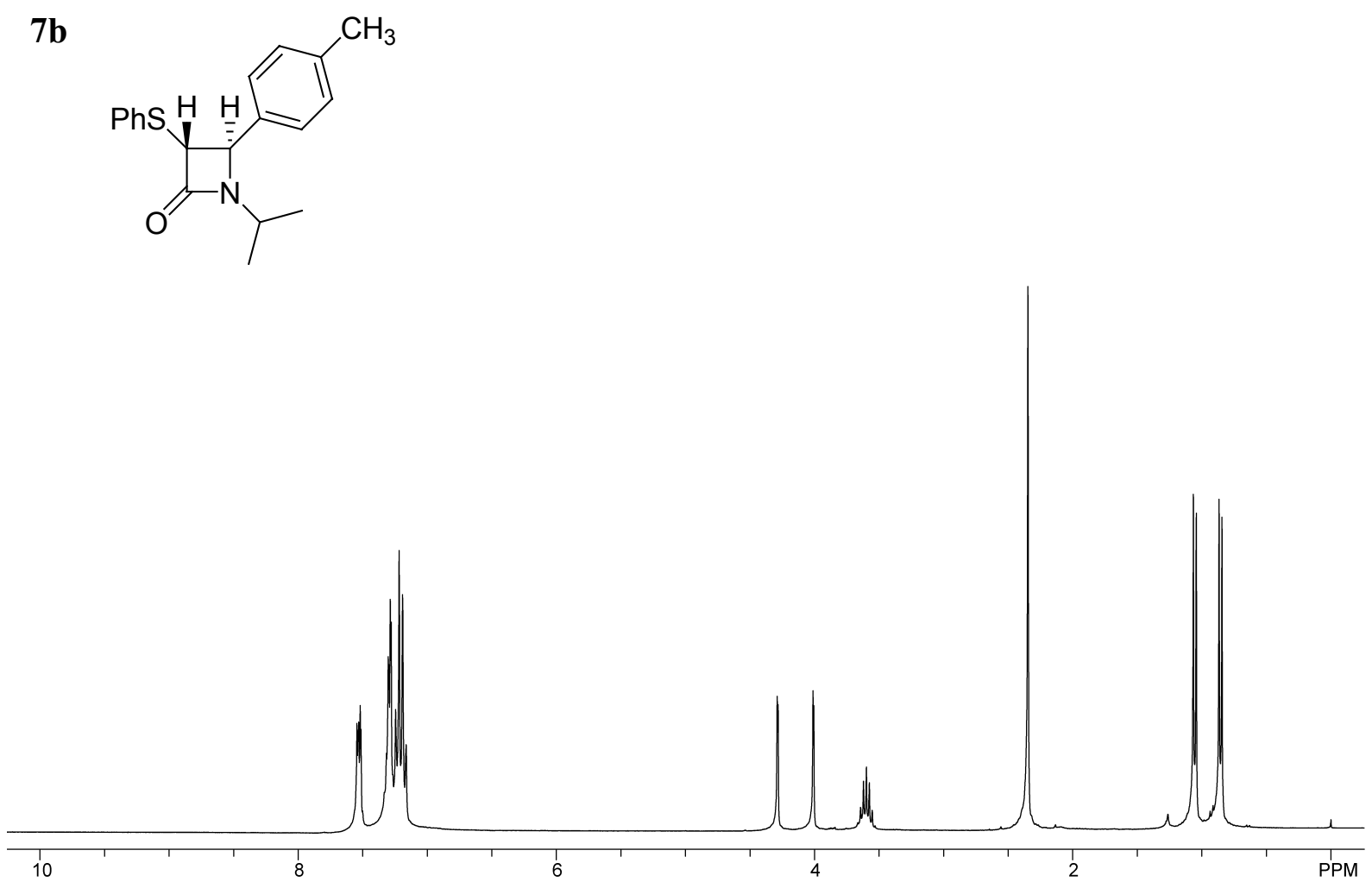
6d

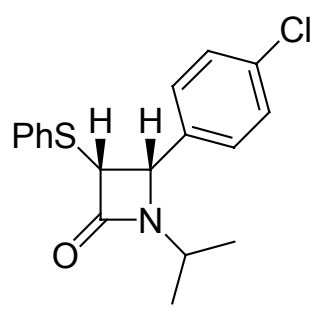

(containing some 7d)

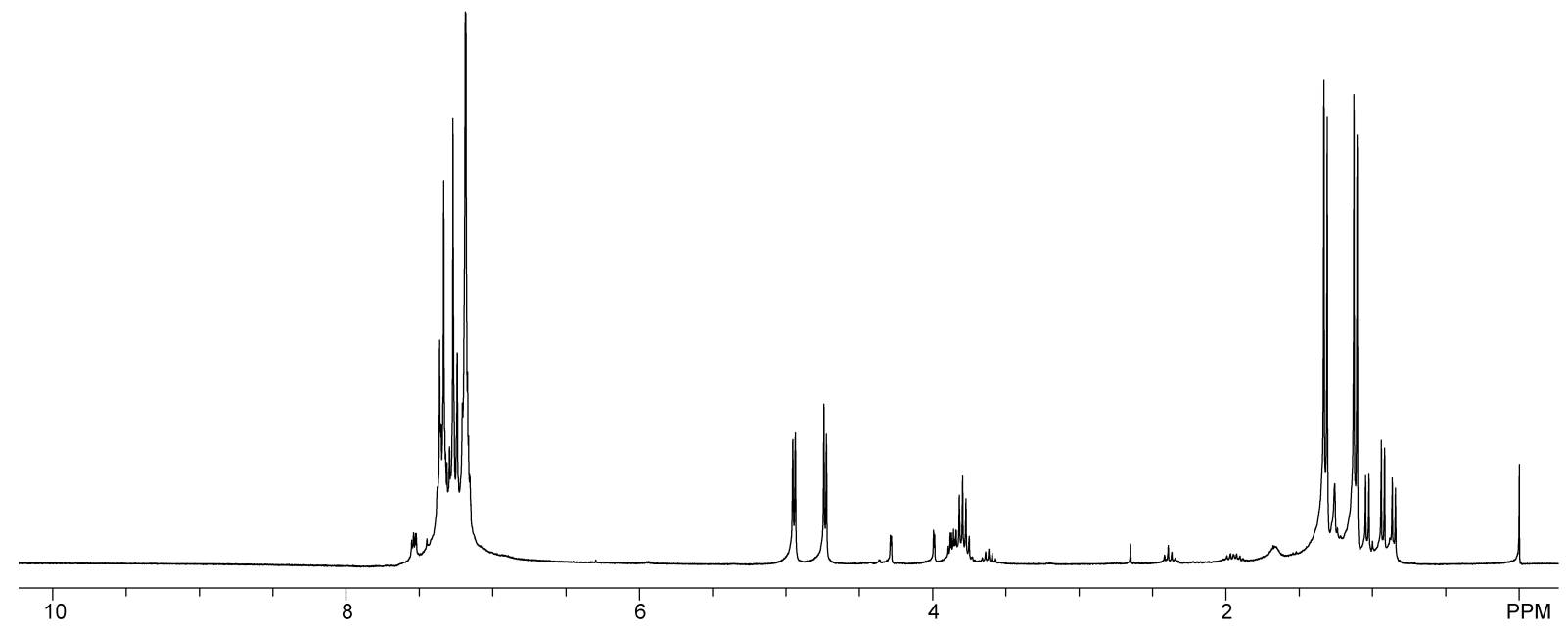

7d<smiles>CC(C)N1C(=O)[C@]([PH3+])(c2ccccc2)[C@H]1c1ccc(Cl)cc1</smiles>

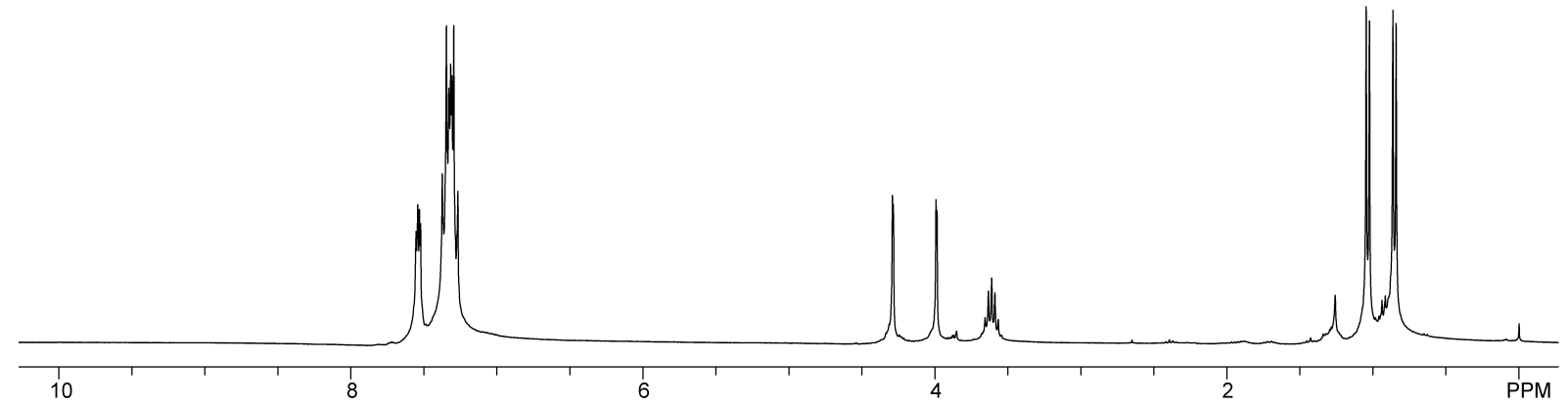


Supplemental Materials

S36

$6 e$
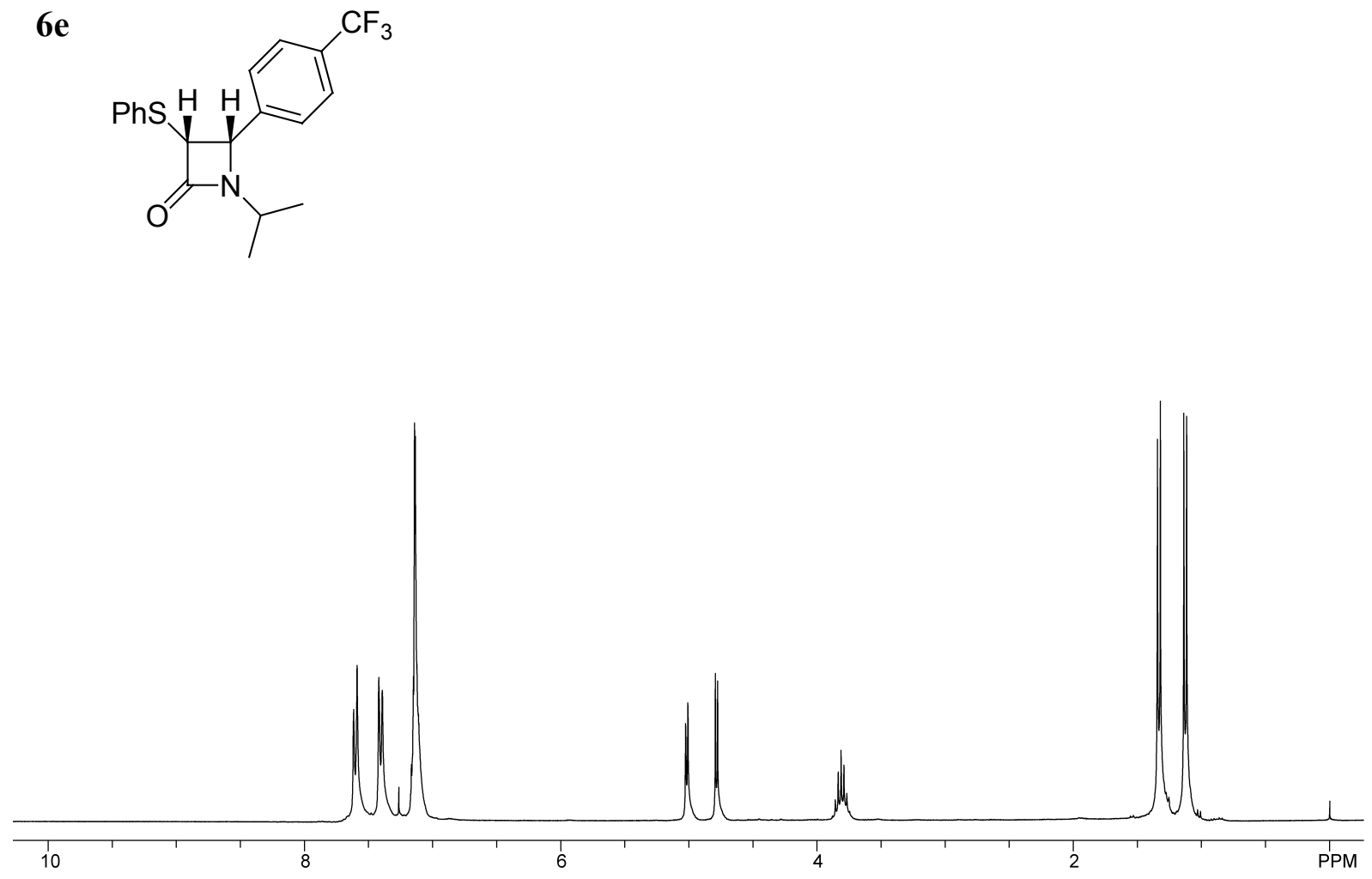

$7 e$
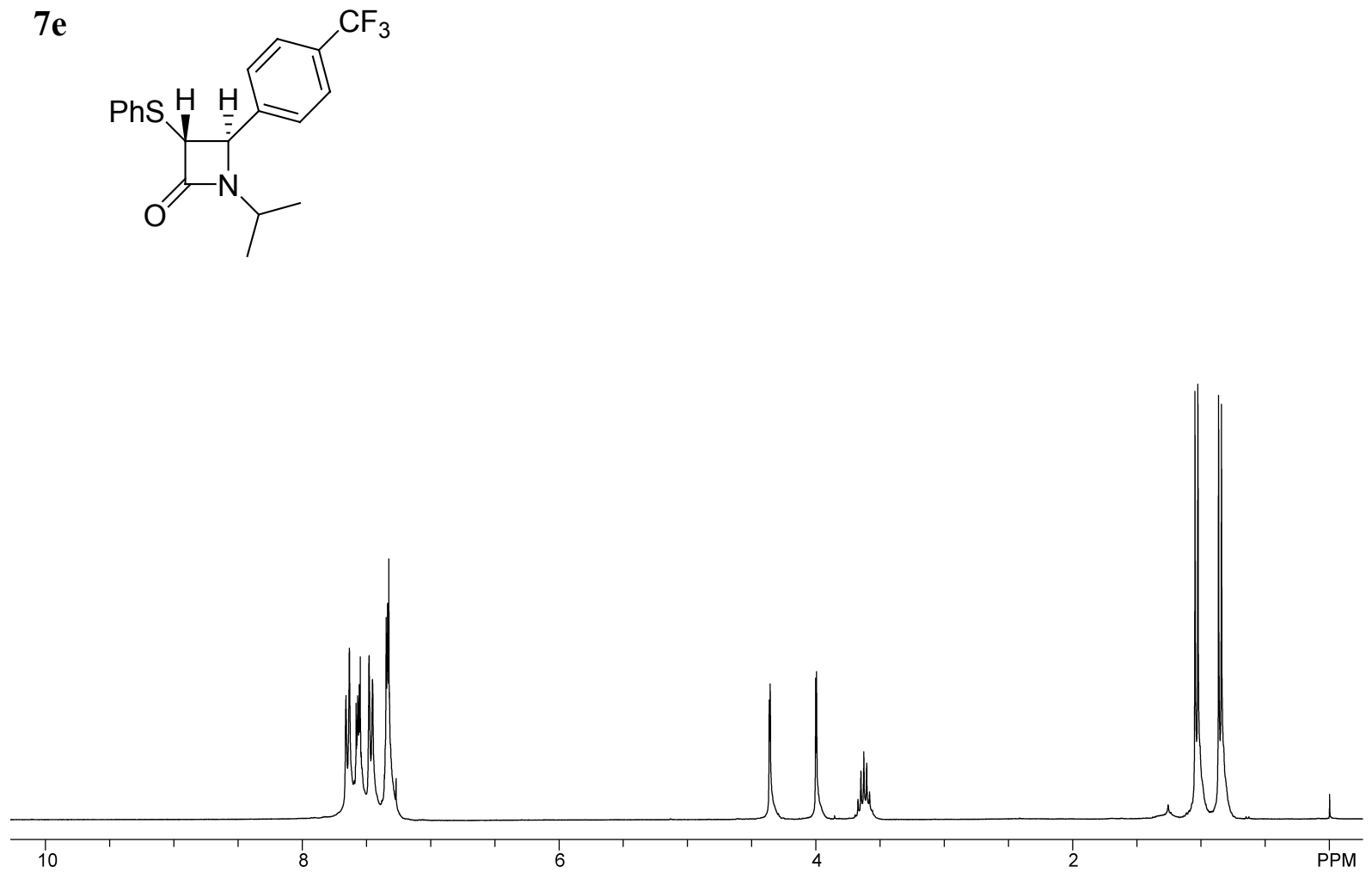

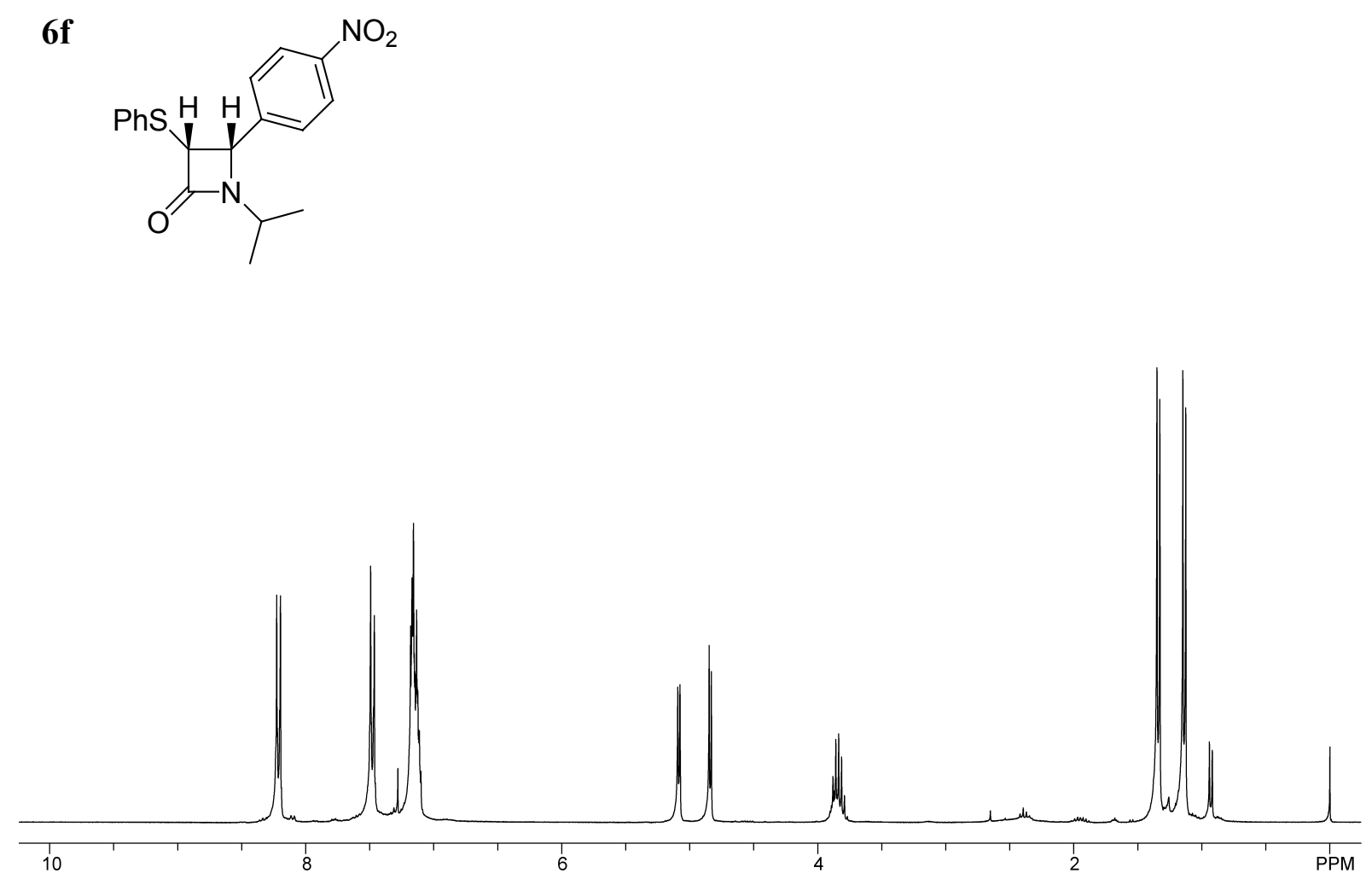

7f<smiles>CC(C)N1C(=O)[C@]([PH3+])(c2ccccc2)[C@H]1c1ccc([N+](=O)[O-])cc1</smiles>

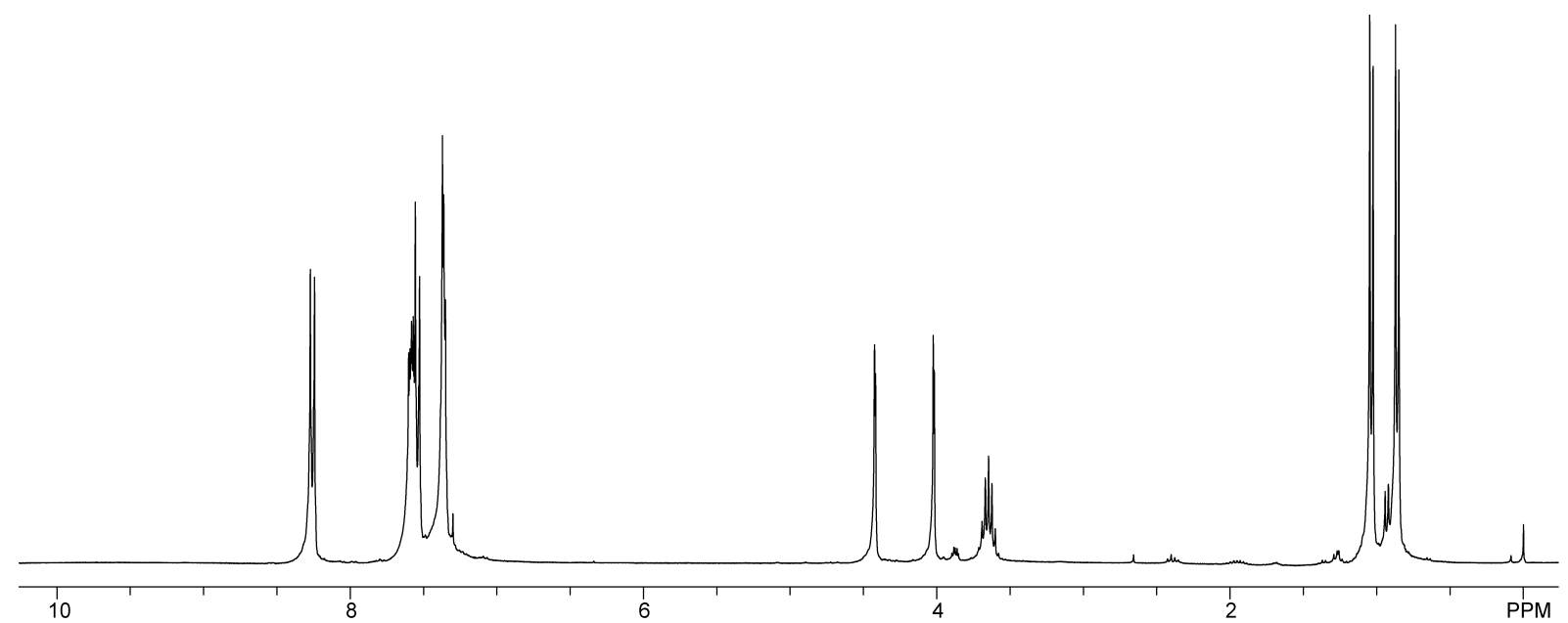




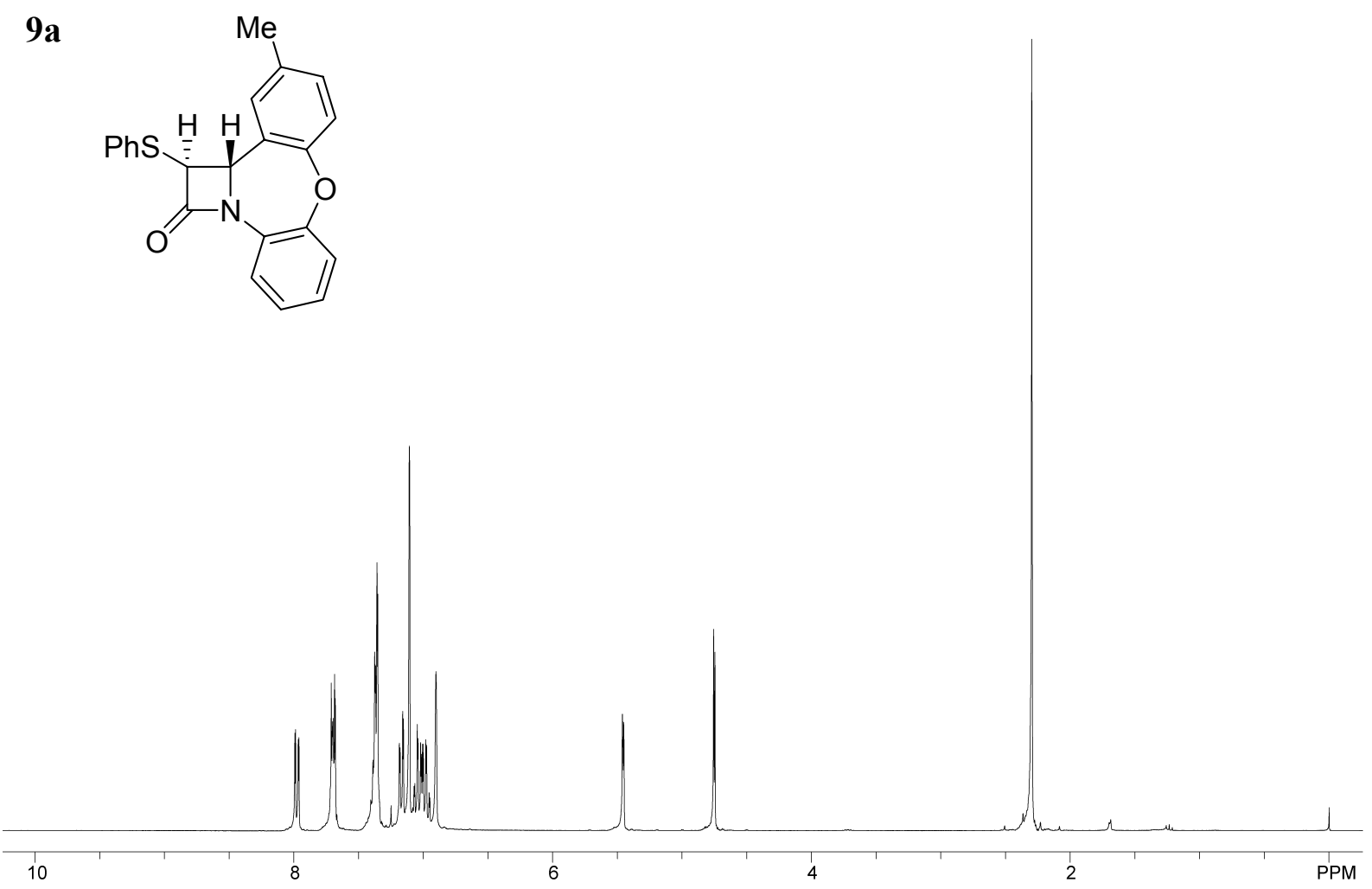

9b
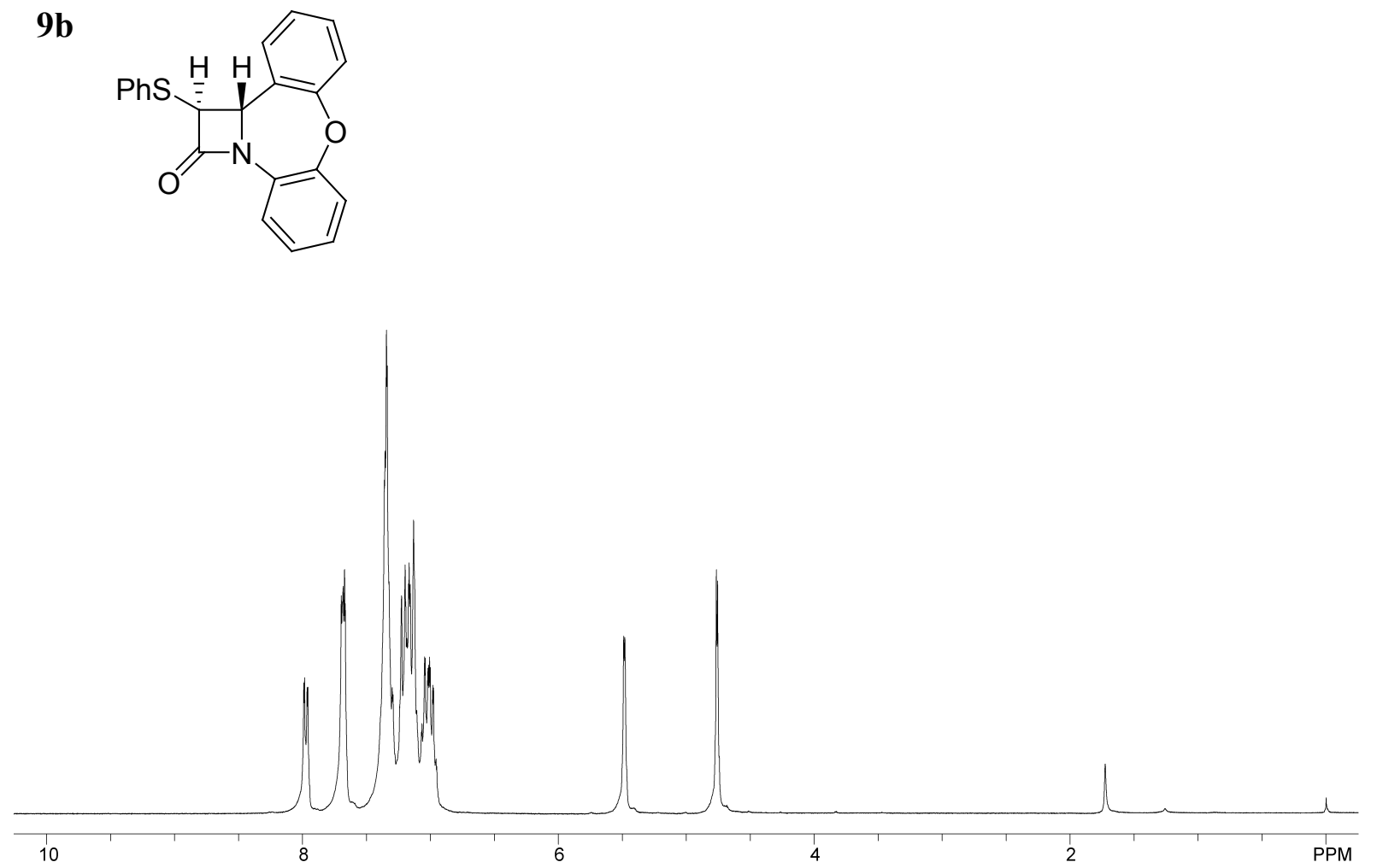

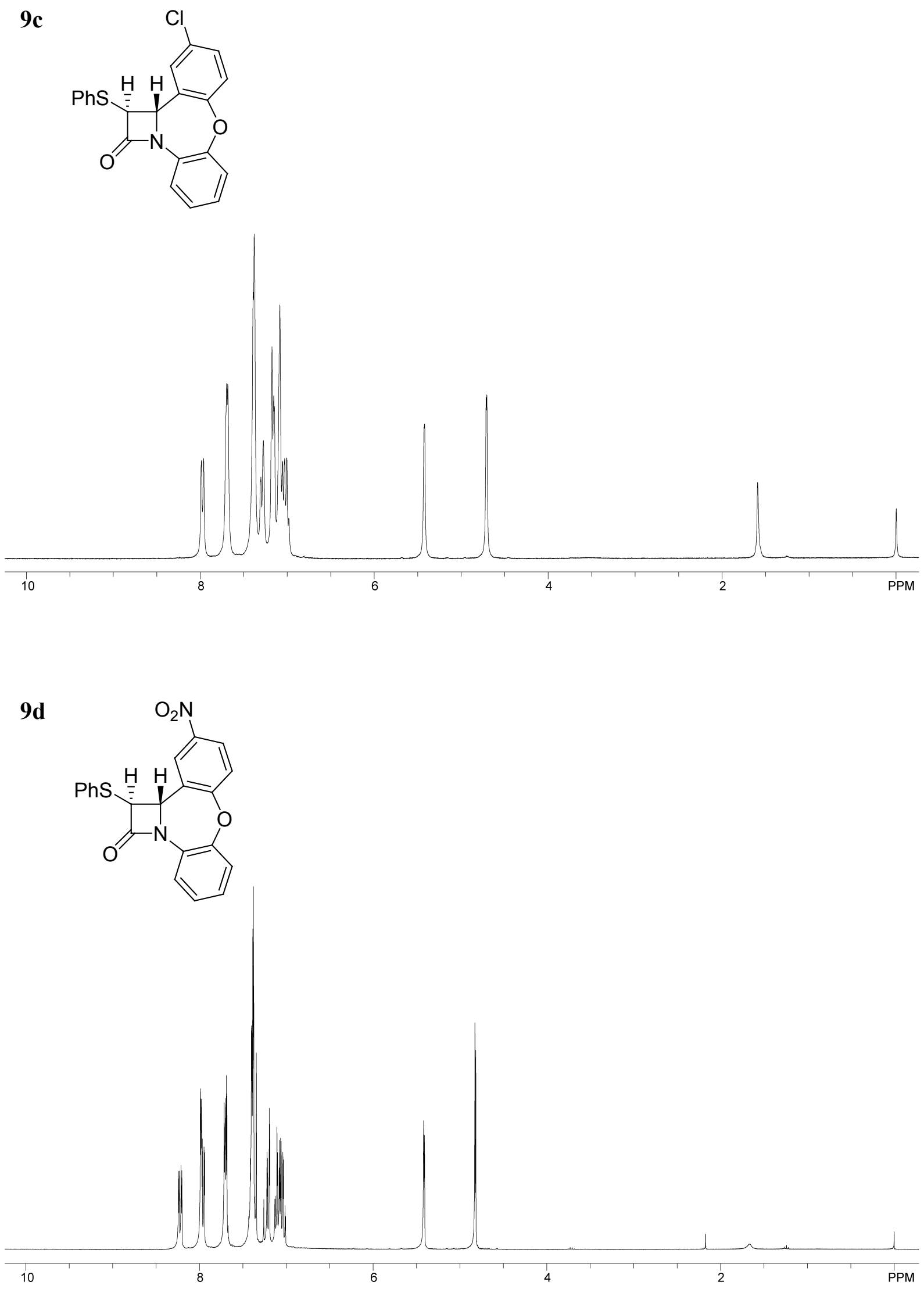


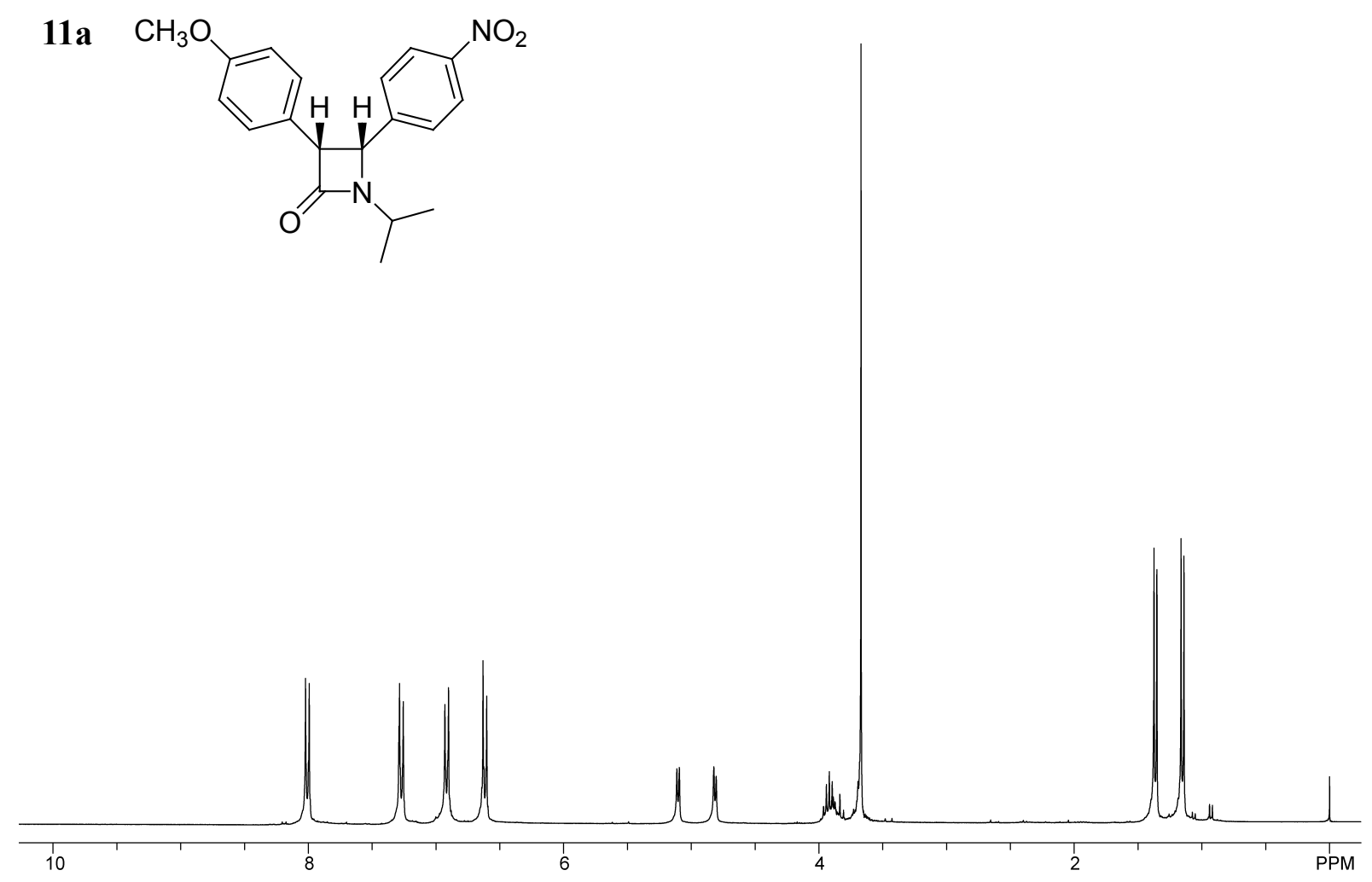

$12 \mathrm{a}$

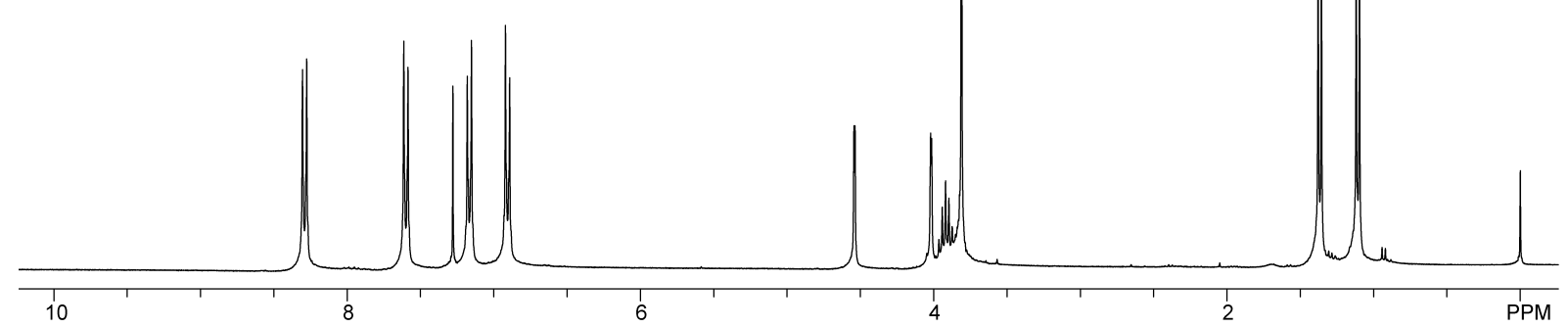



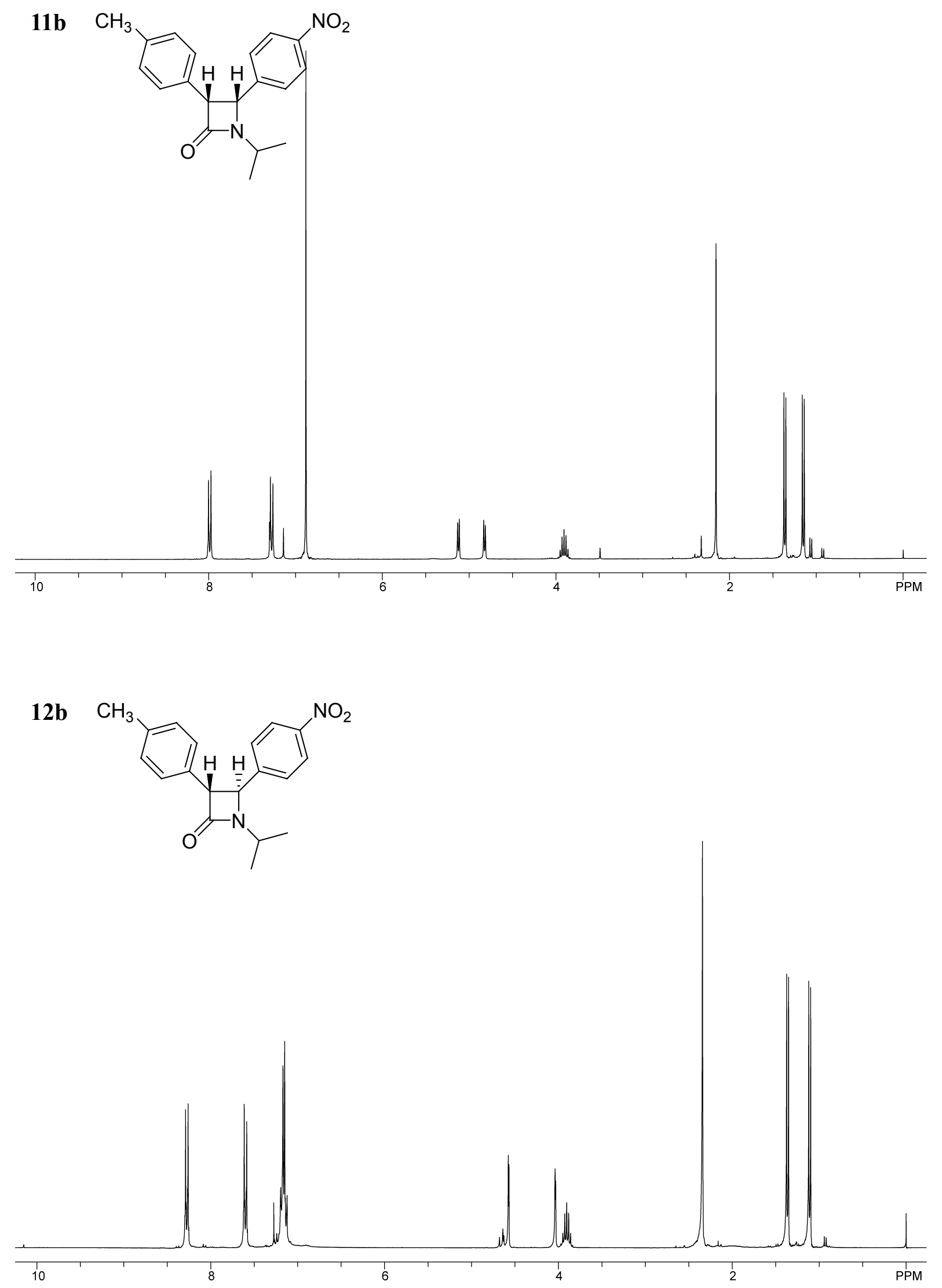
$11 \mathrm{c}$<smiles>CC(C)N1C(=O)[C@H](c2ccccc2)[C@@H](c2ccc([N+](=O)[O-])cc2)[C@H]1c1ccccc1</smiles>

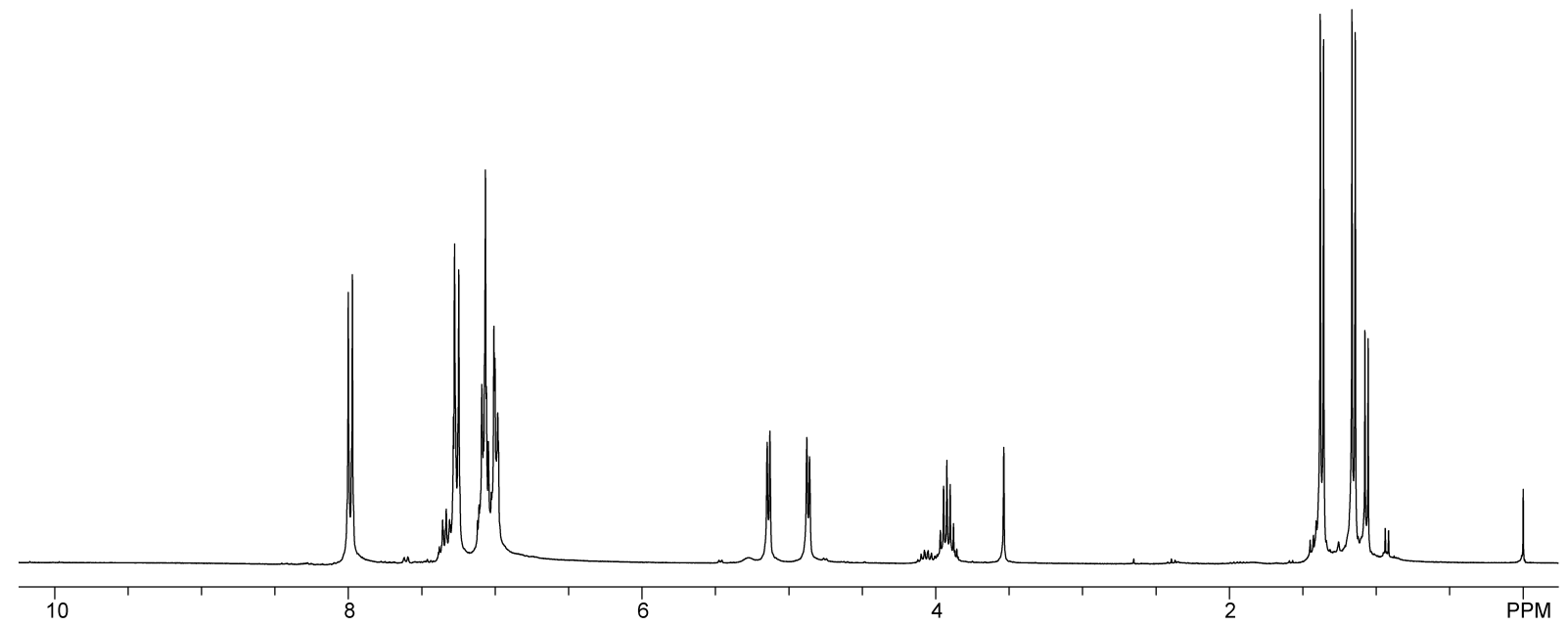

$12 c$<smiles>CC(C)N1C(=O)[C@H](c2ccccc2)[C@@H]1c1ccc([N+](=O)[O-])cc1</smiles>

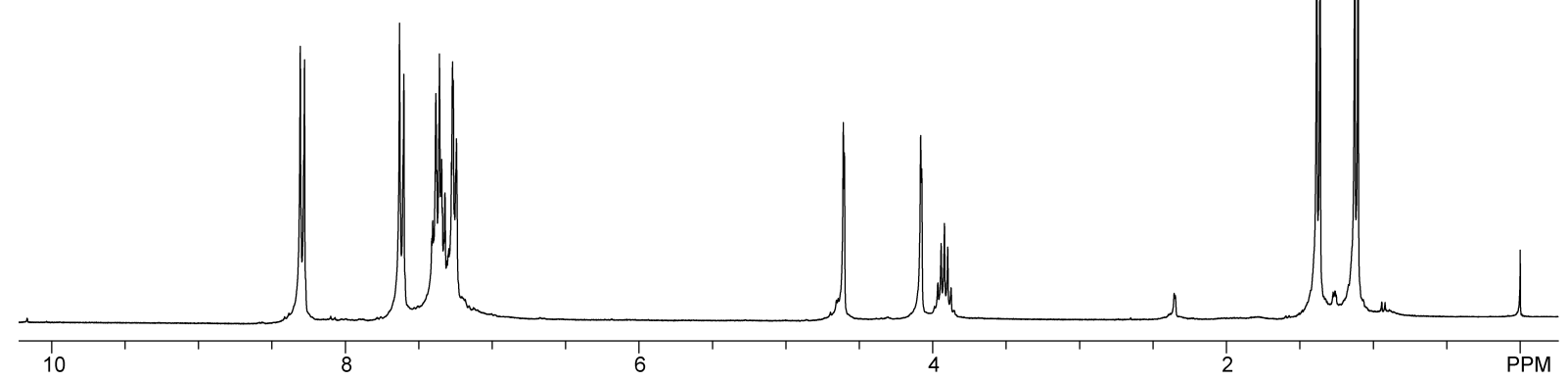


$11 \mathrm{~d}$

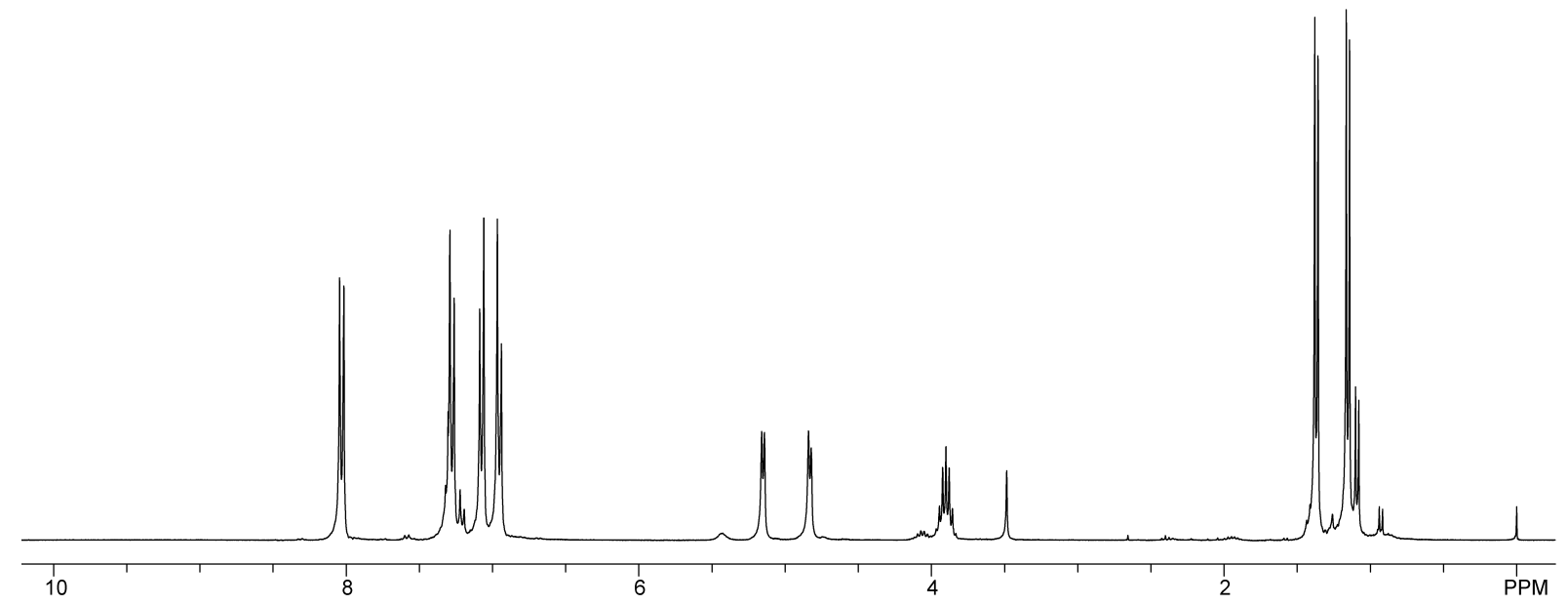

12d $\mathrm{Cl}$<smiles>CC(C)N1C(=O)[C@H](c2ccc(Cl)cc2)[C@H]1c1ccc([N+](=O)[O-])cc1</smiles>

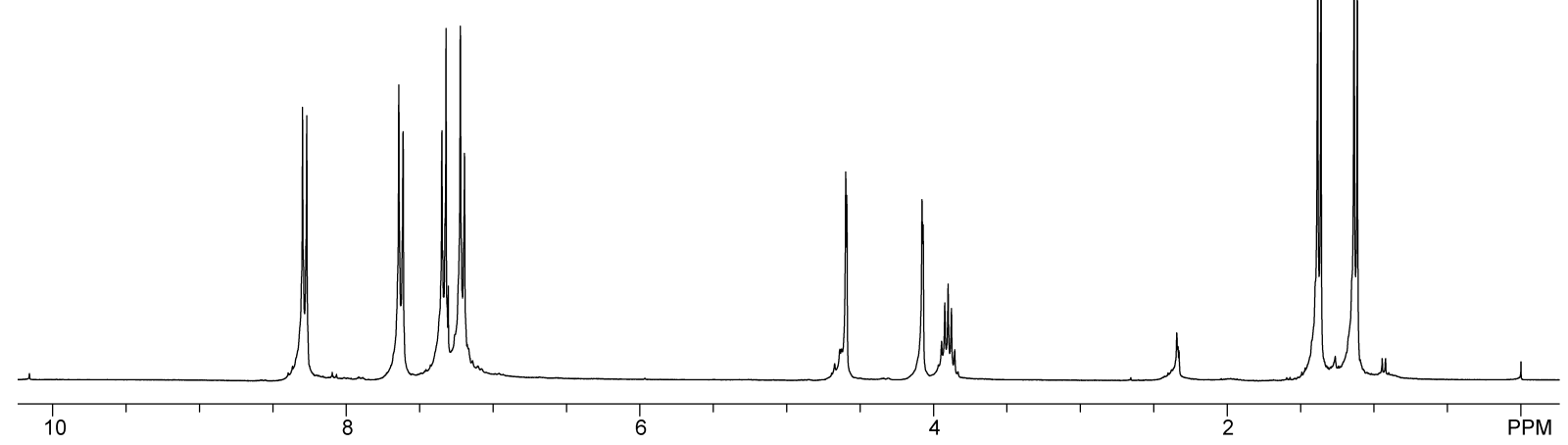


$11 \mathrm{e}$

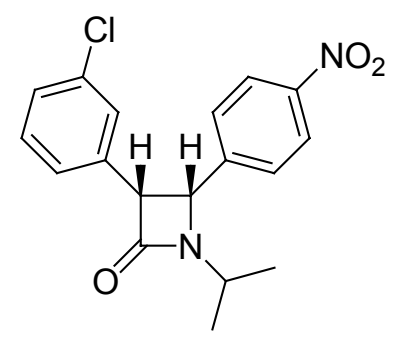

(containing some byproduct 2-(3-chlorophenyl)- $\mathrm{N}$-isopropylacetamide)

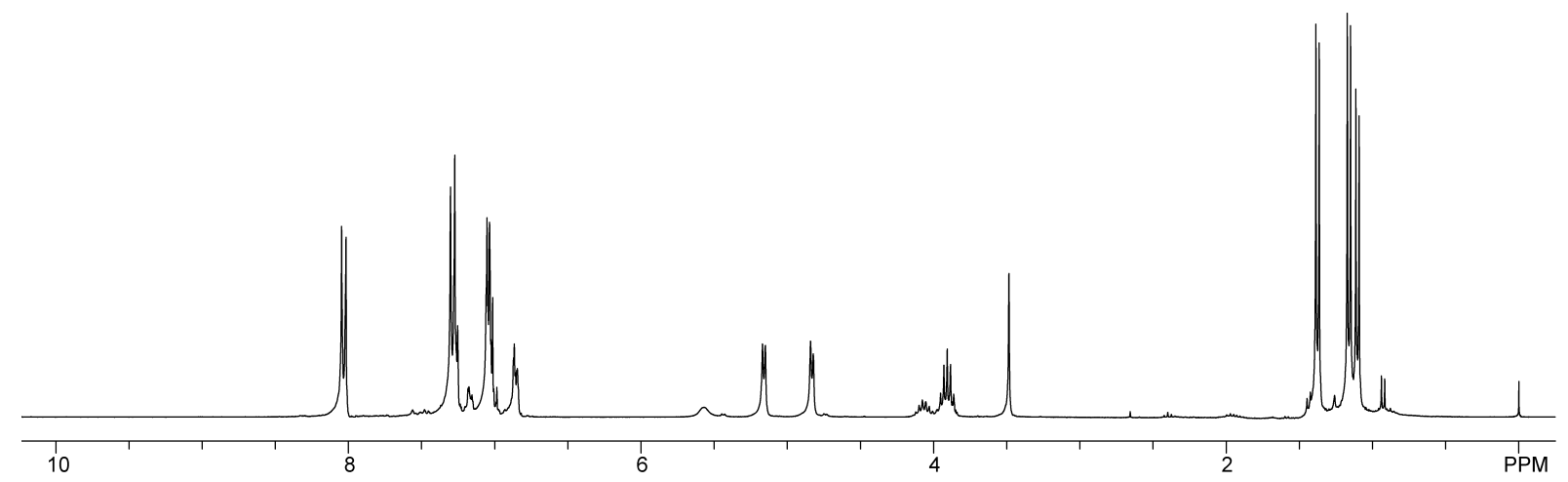

$12 \mathrm{e}$<smiles>CC(C)N1C(=O)[C@@H](c2cccc(Cl)c2)[C@@H]1c1ccc([N+](=O)[O-])cc1</smiles>

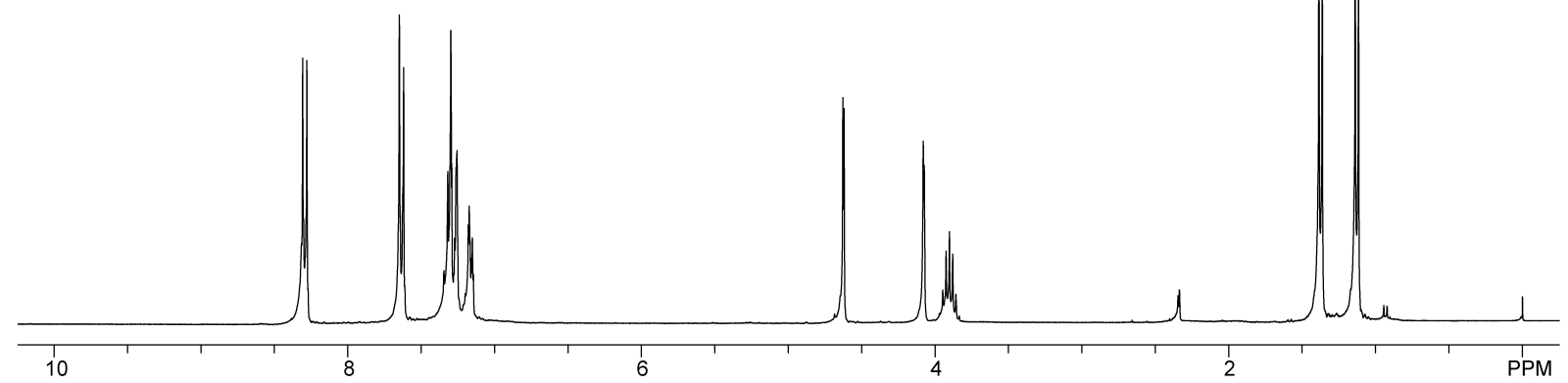




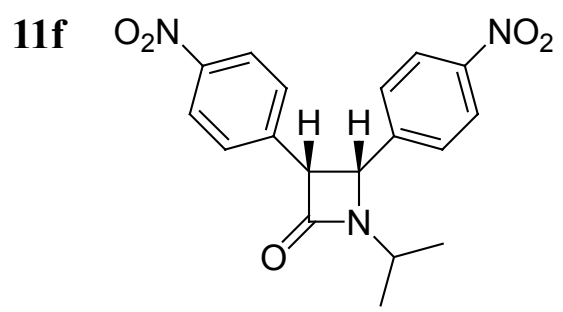

(containing some byproduct 2-(4-nitrophenyl)- $N$-isopropylacetamide)

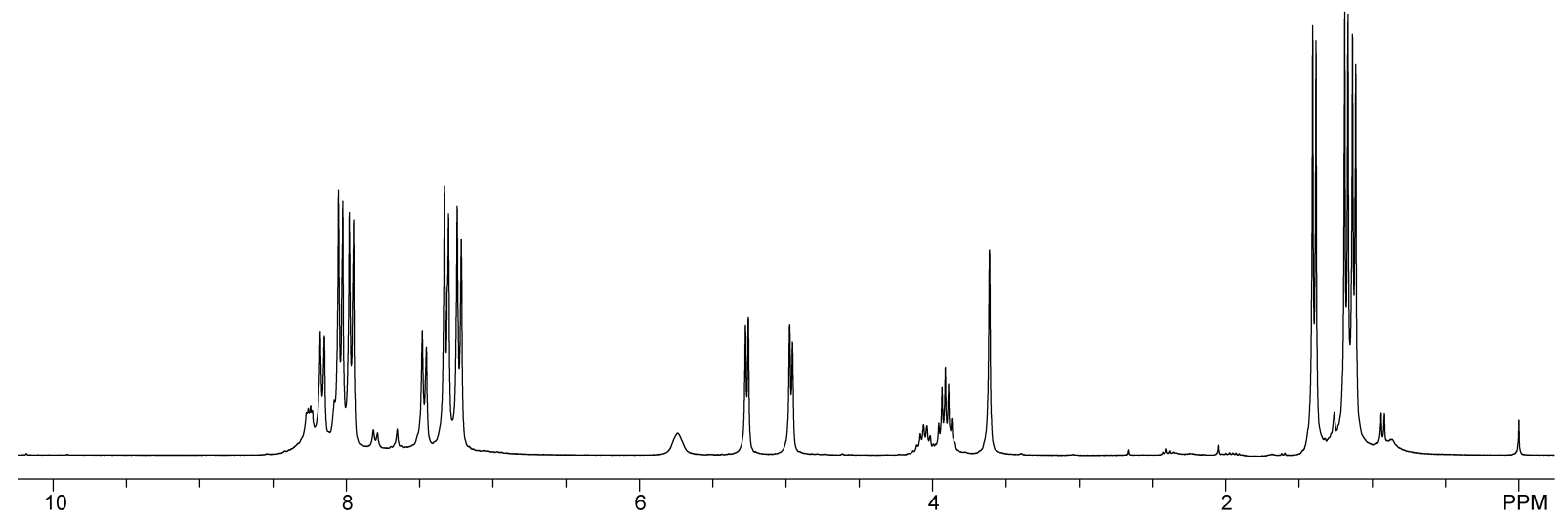<smiles>CCCO[N+](=O)[O-]</smiles>

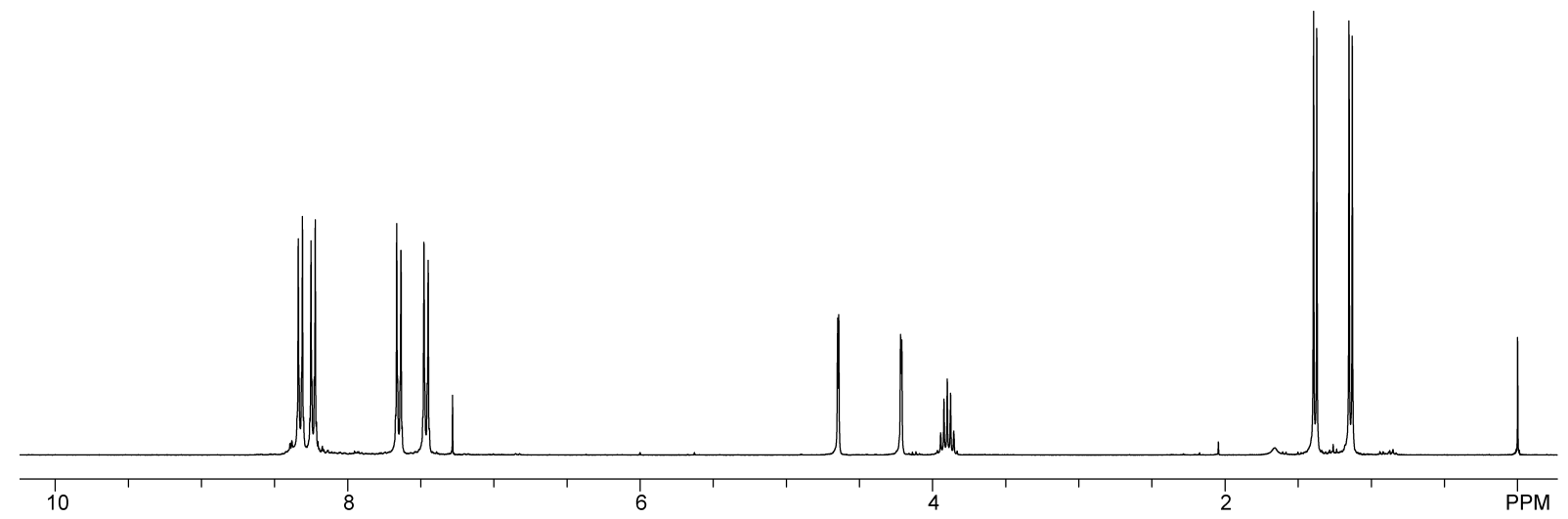



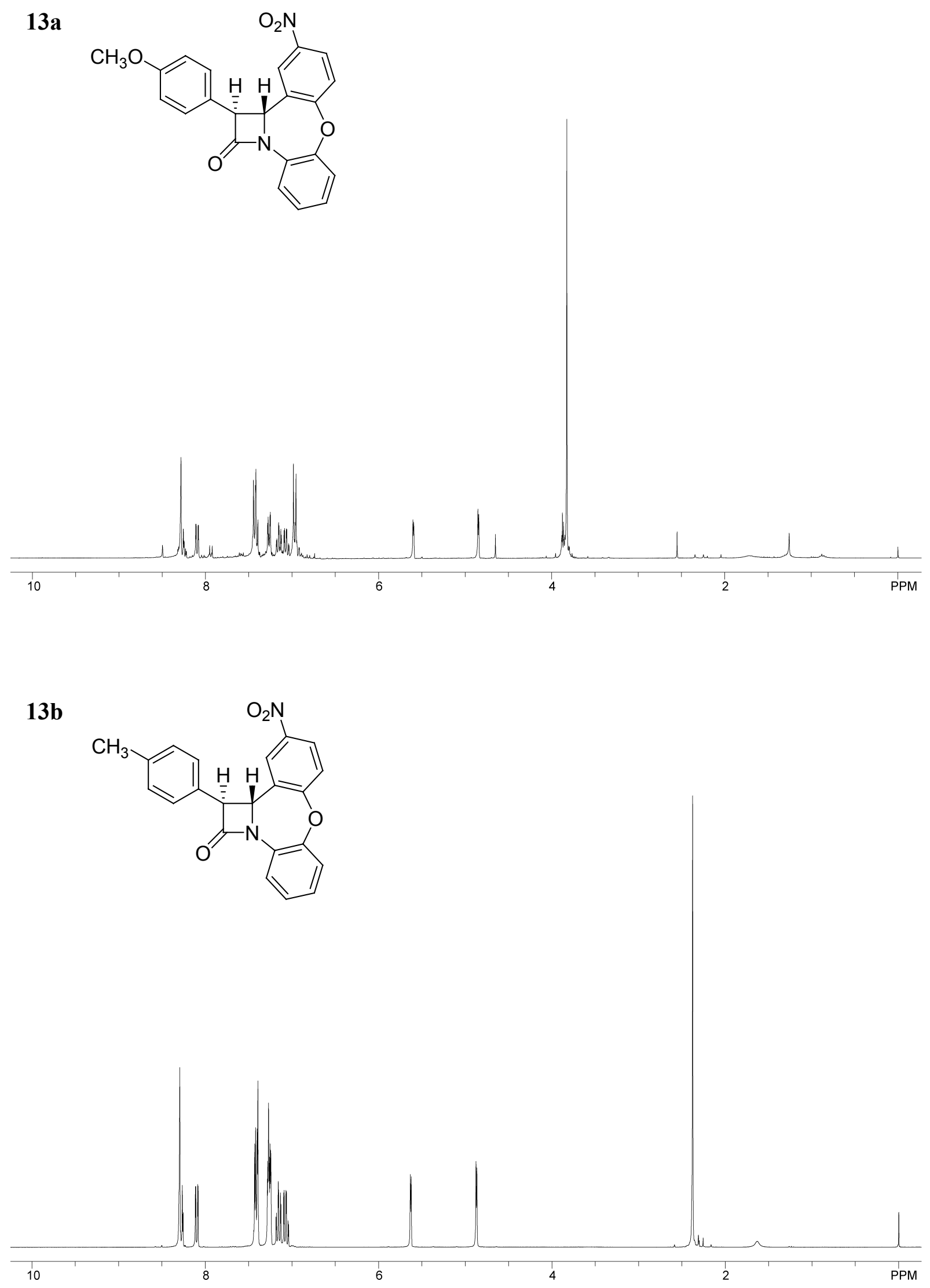

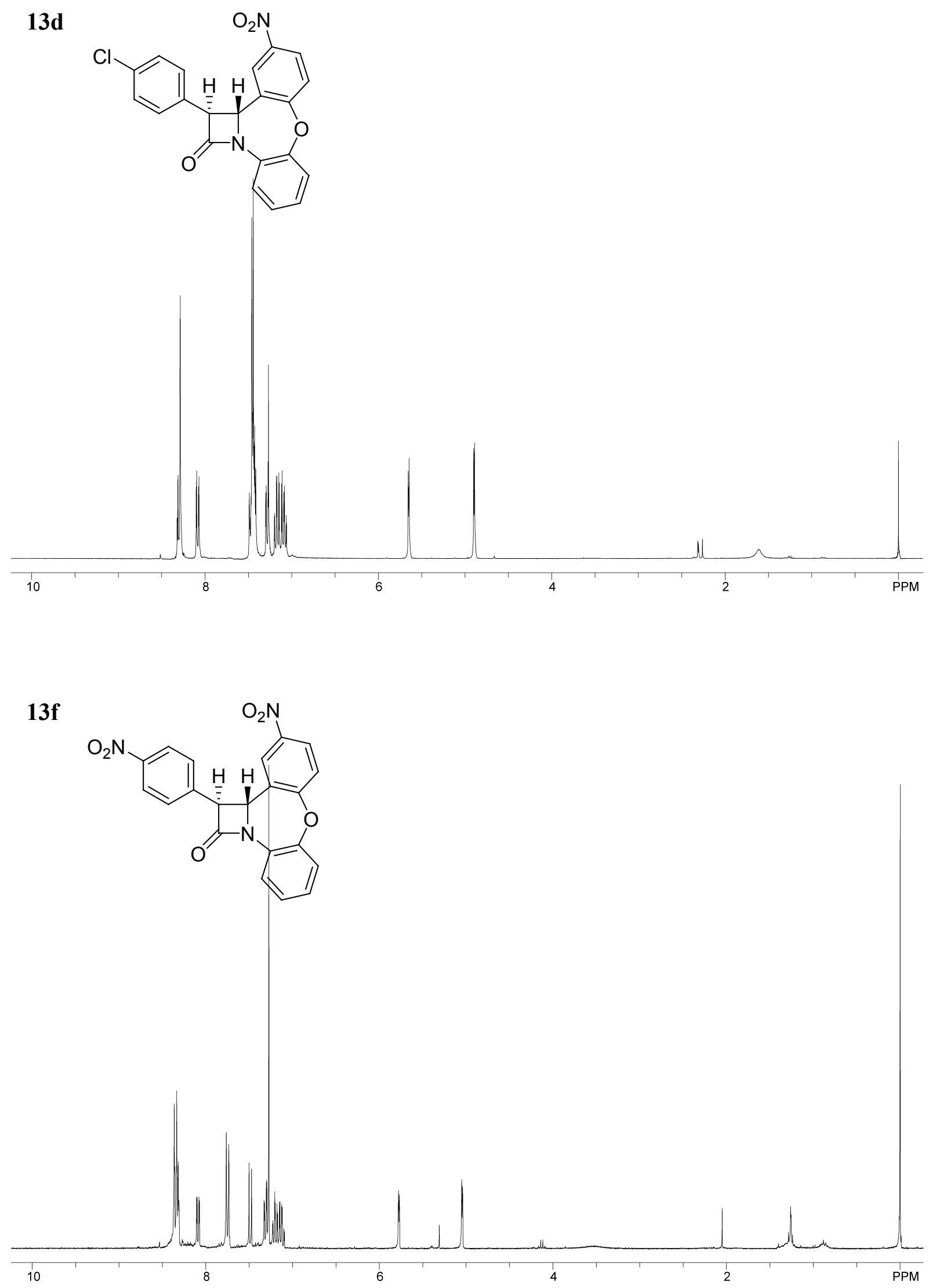

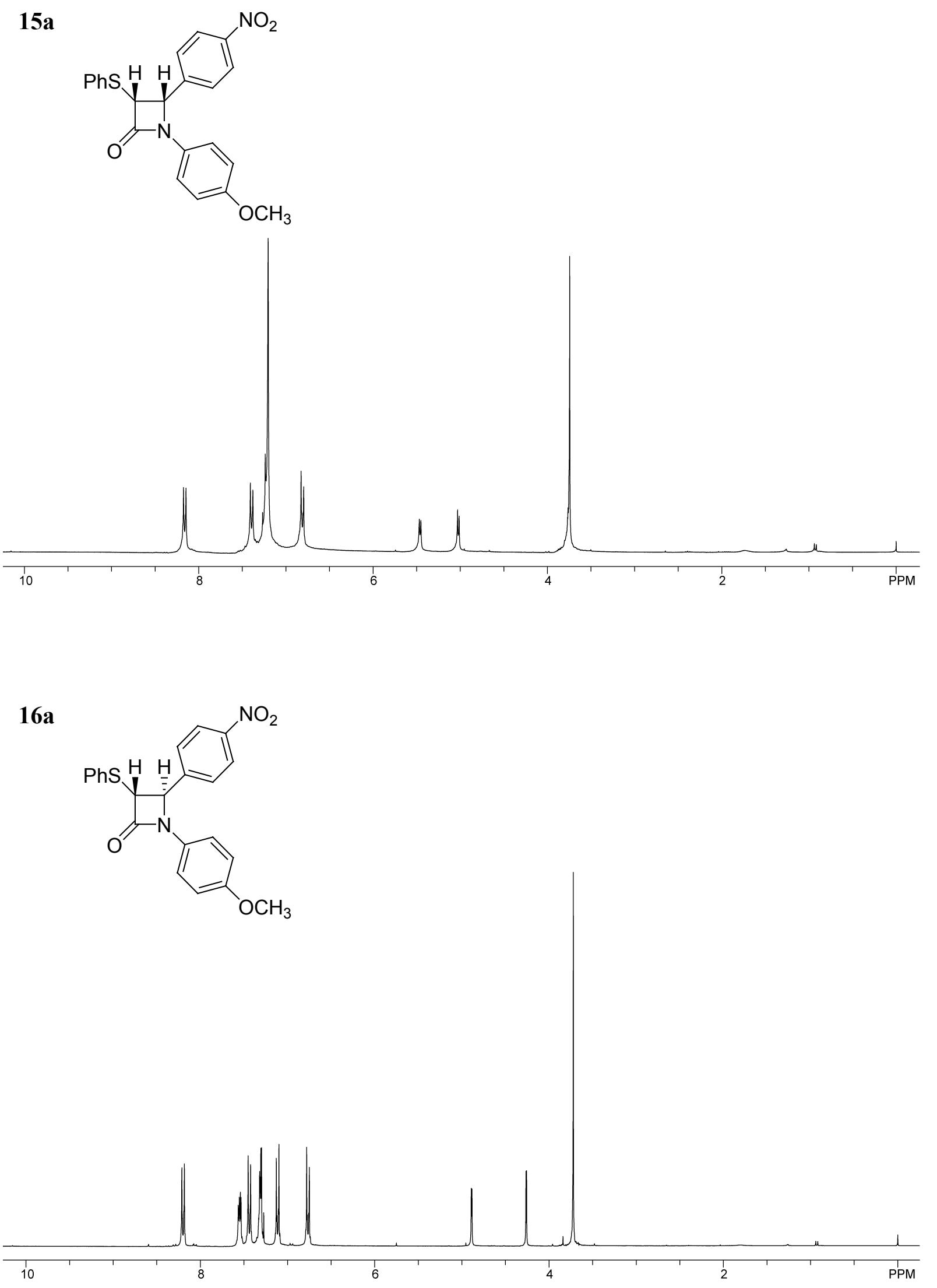

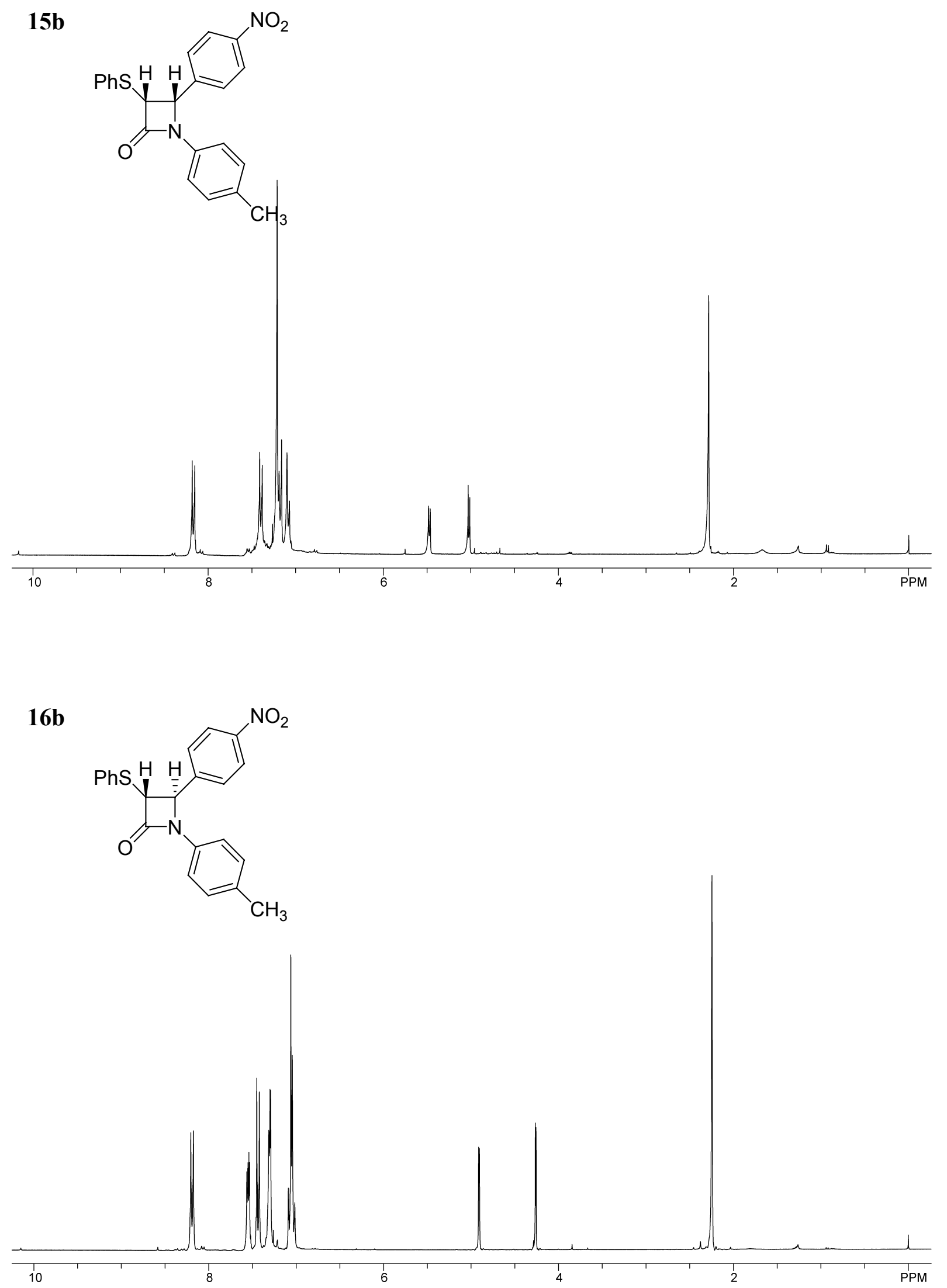

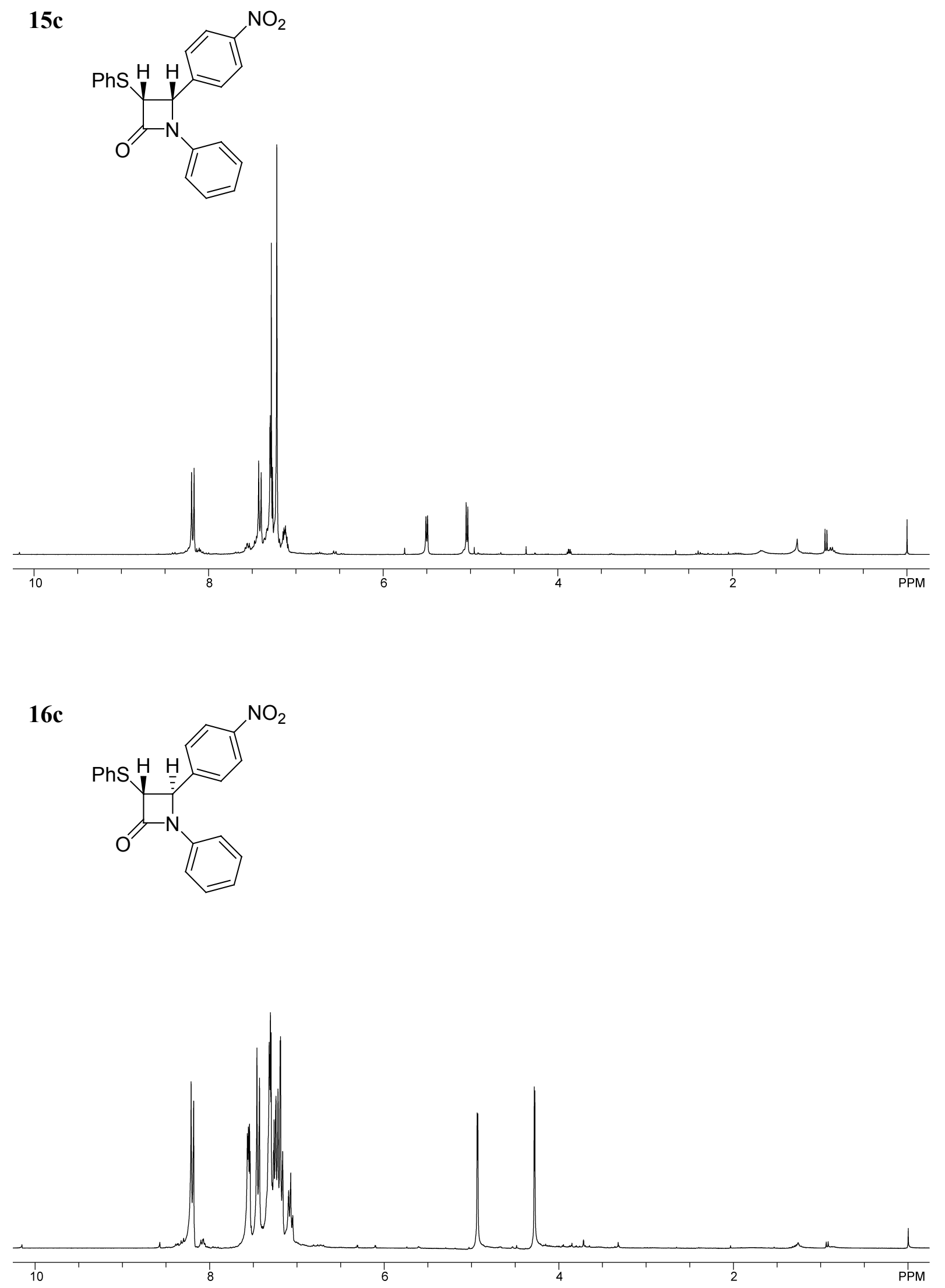

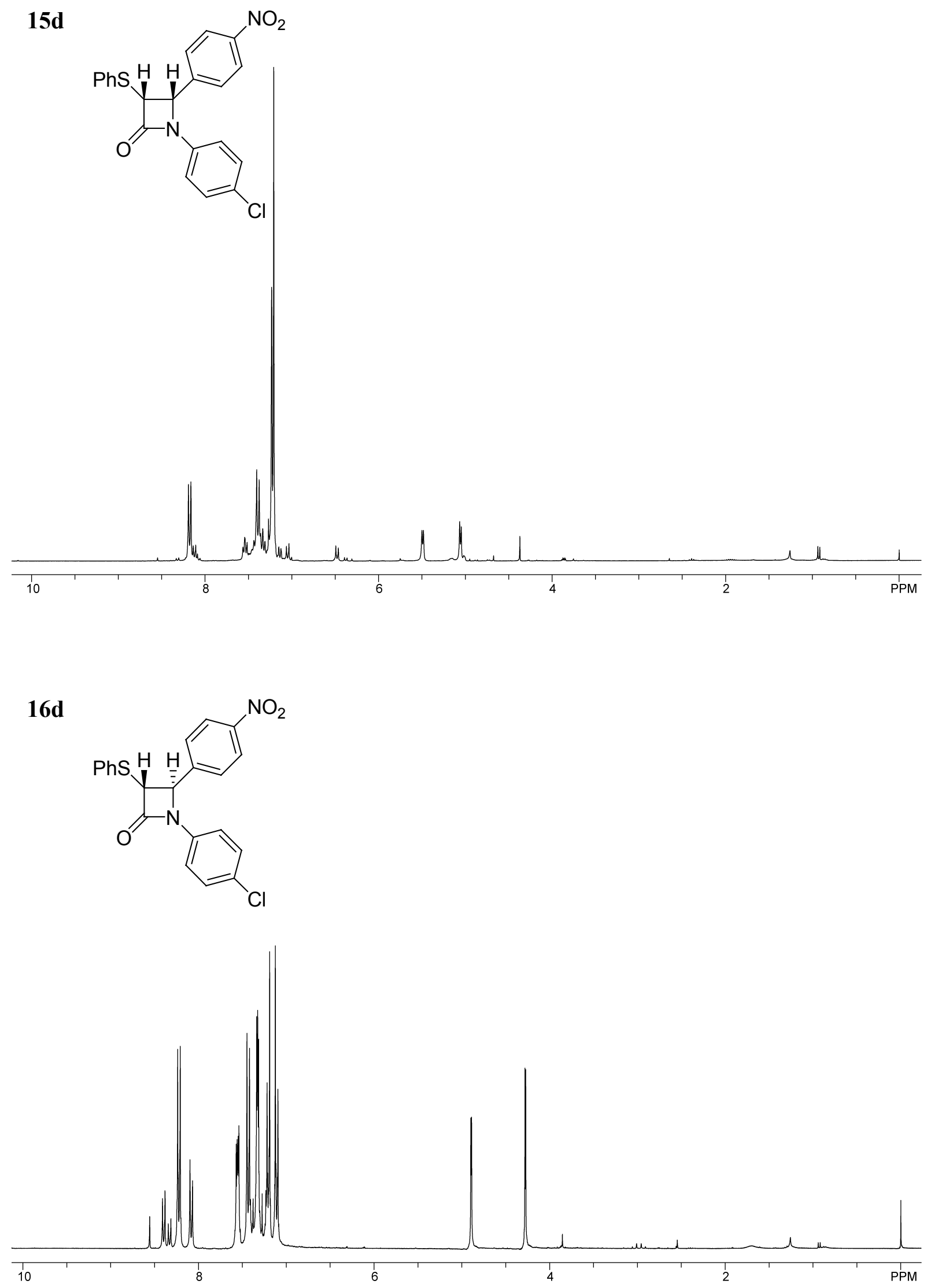


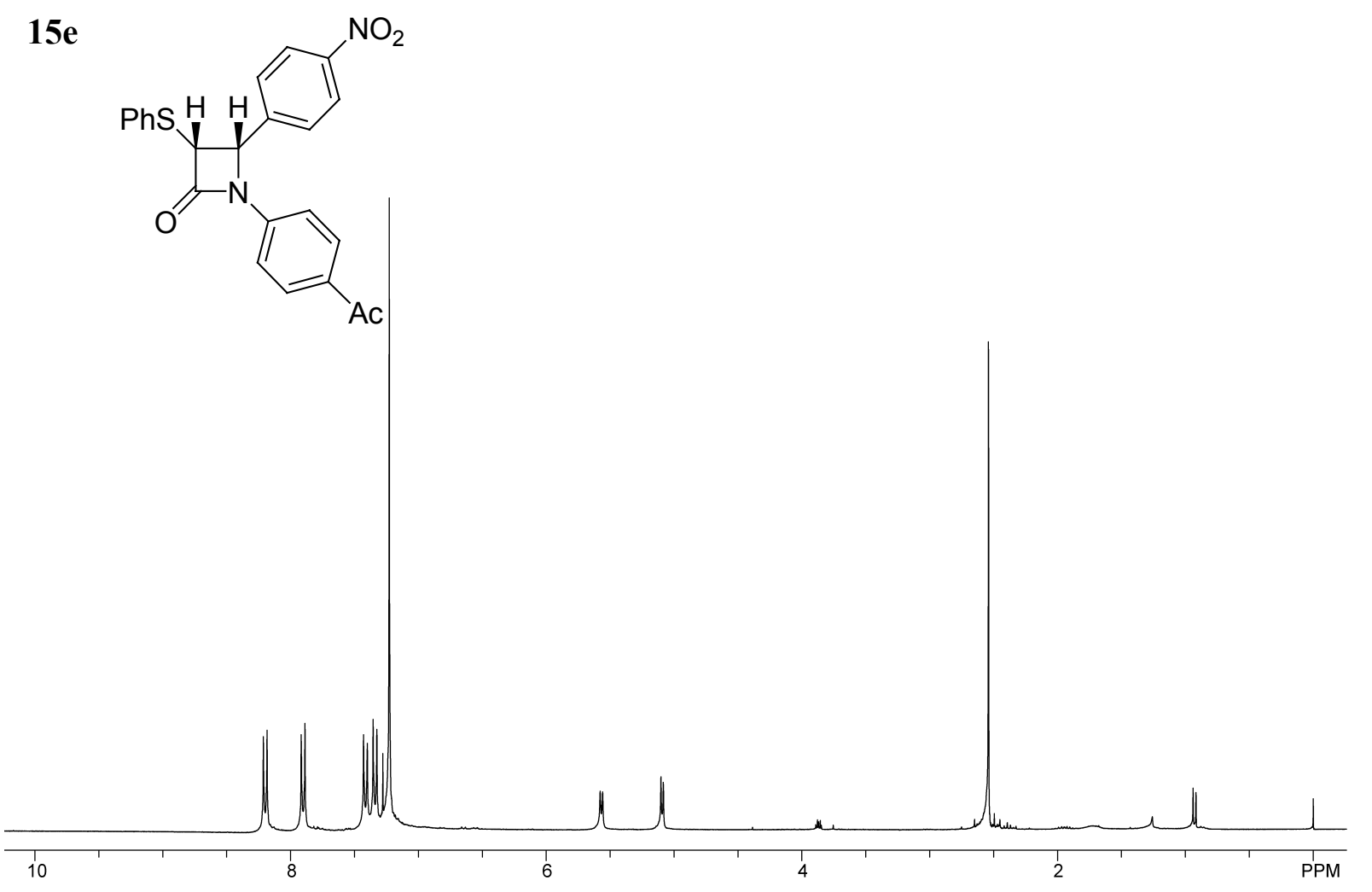

$16 \mathrm{e}$
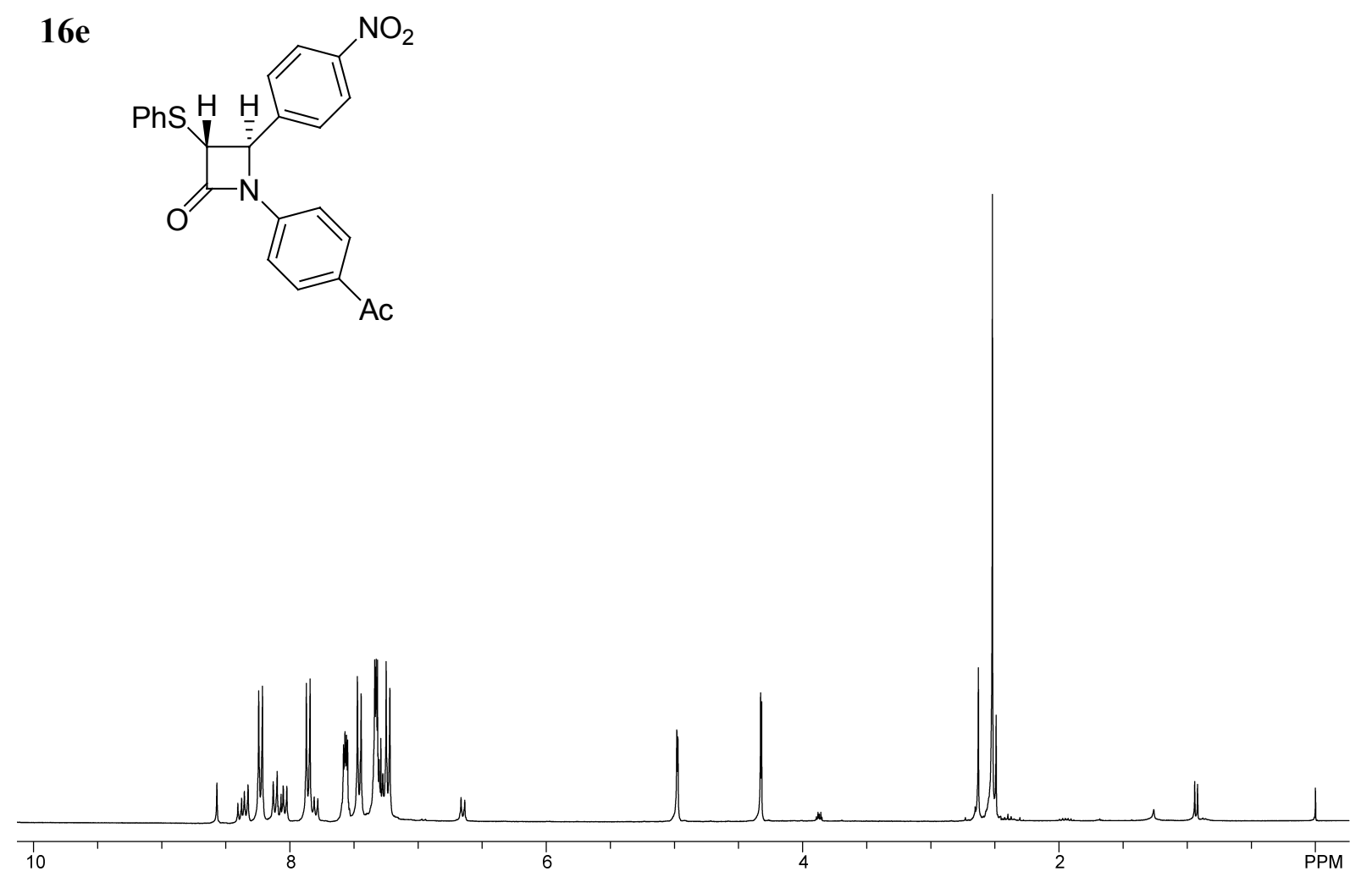
Supplemental Materials

S53

$17 \mathbf{a}$
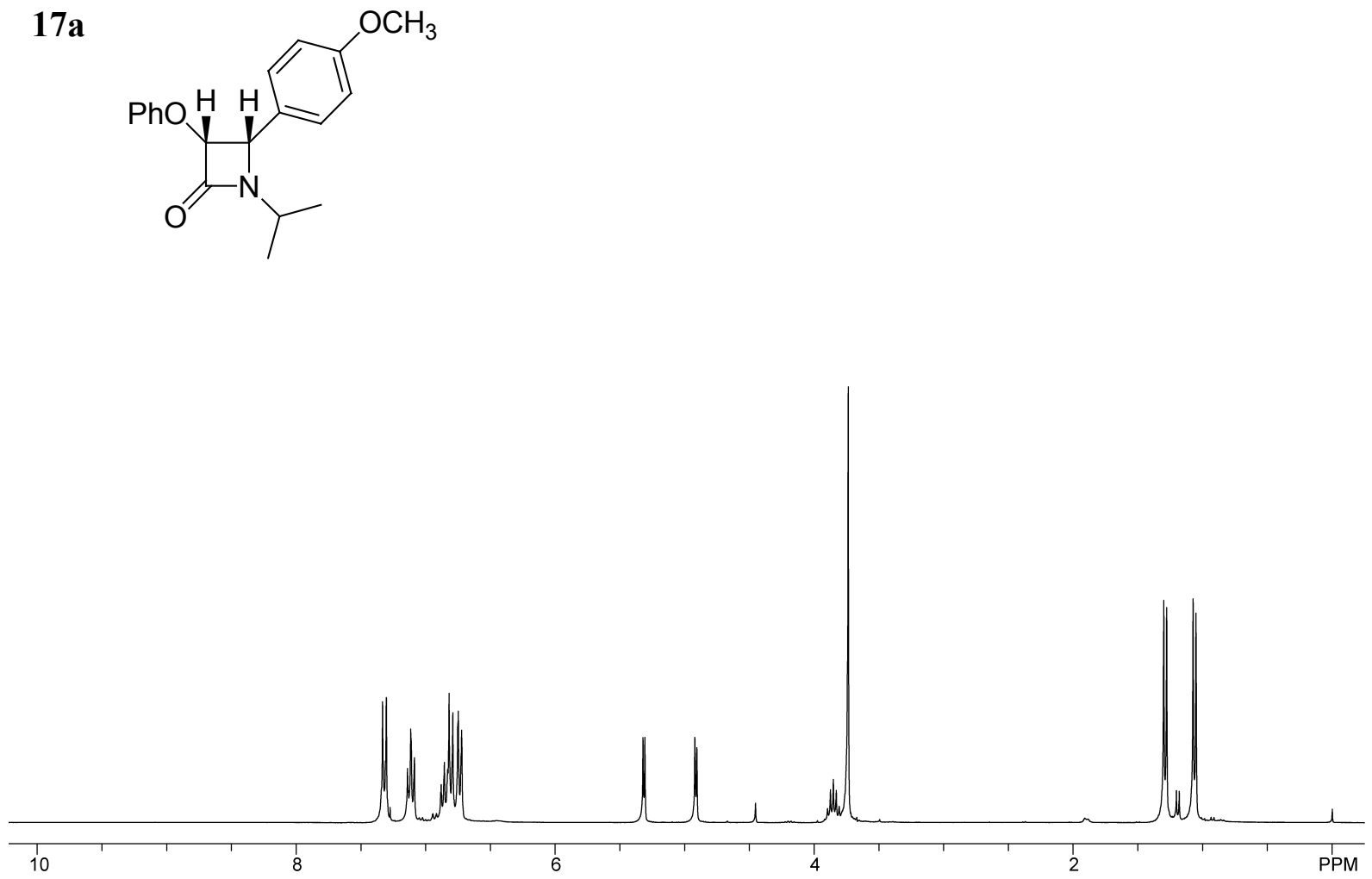

17b

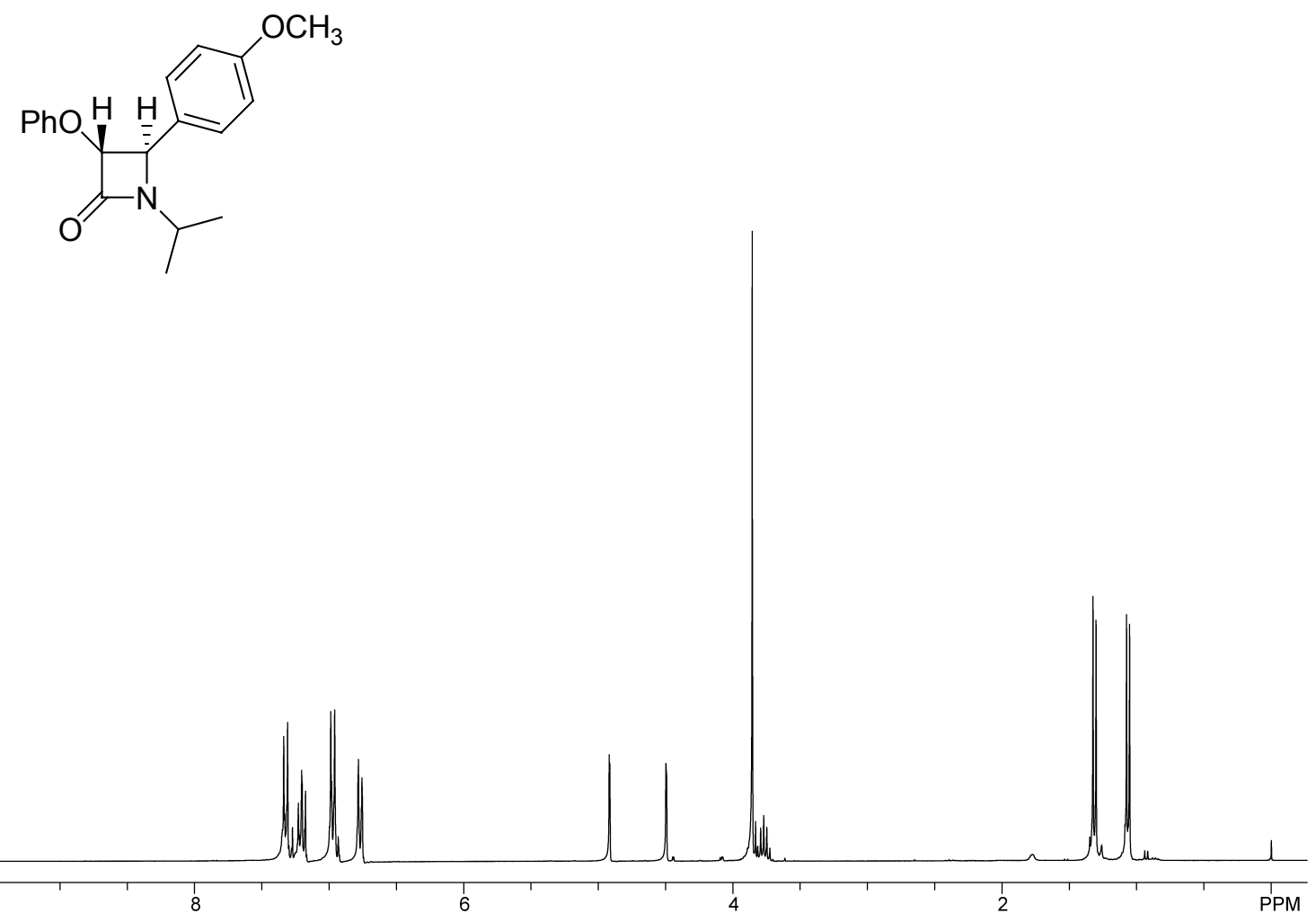




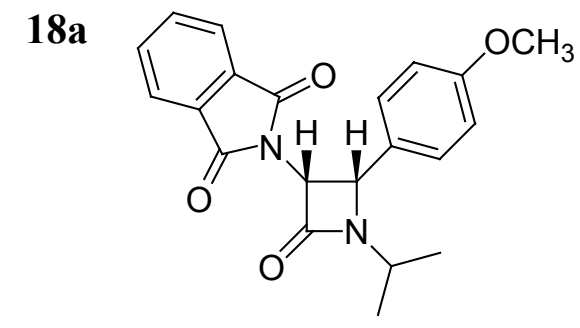

(containing some byproduct $N$-isopropyl-2-(1,3-dioxoisoindolin-2-yl)acetamide)

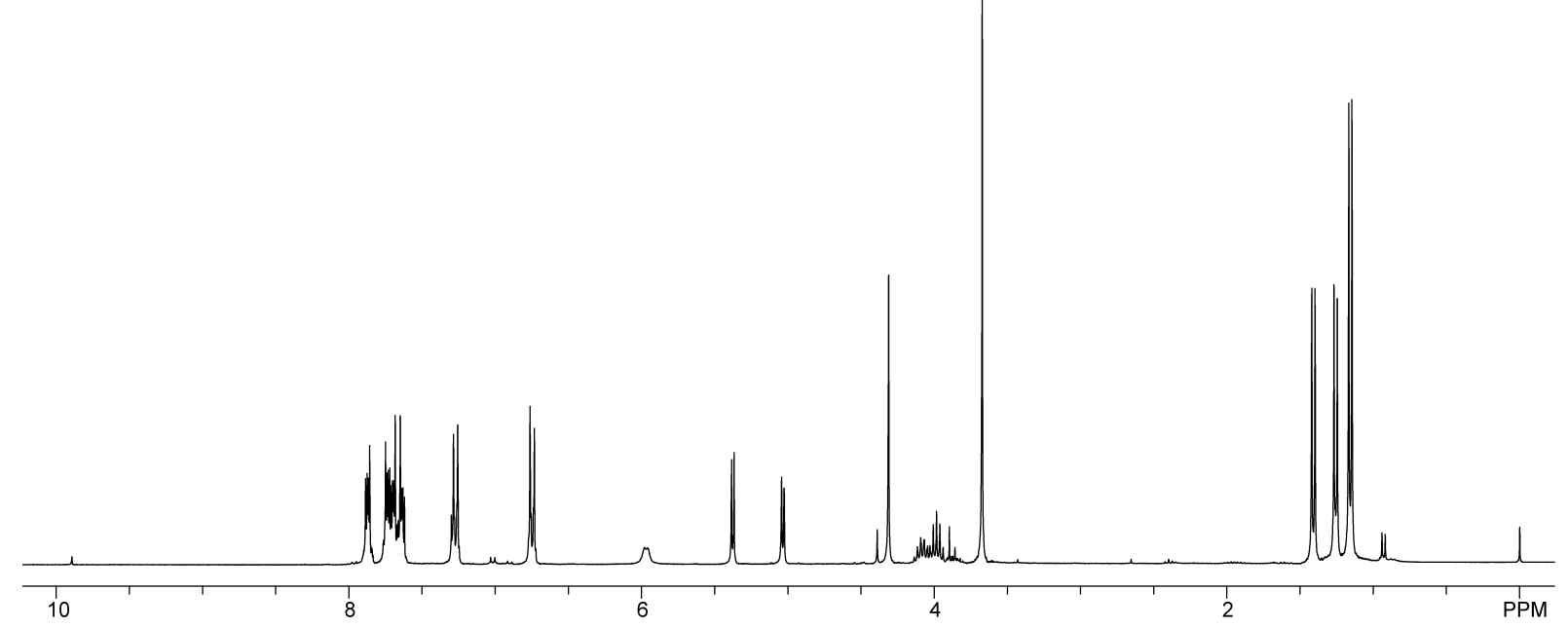<smiles>COc1ccc(C2C(=O)N(C(C)C)C2=O)cc1</smiles>

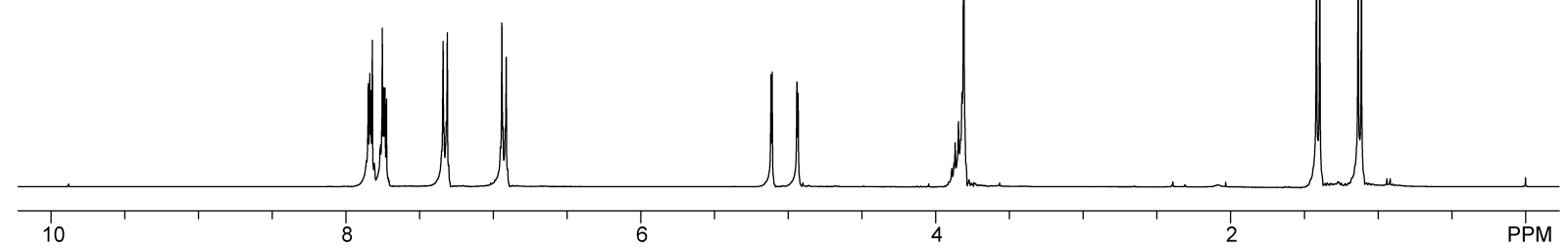


19a
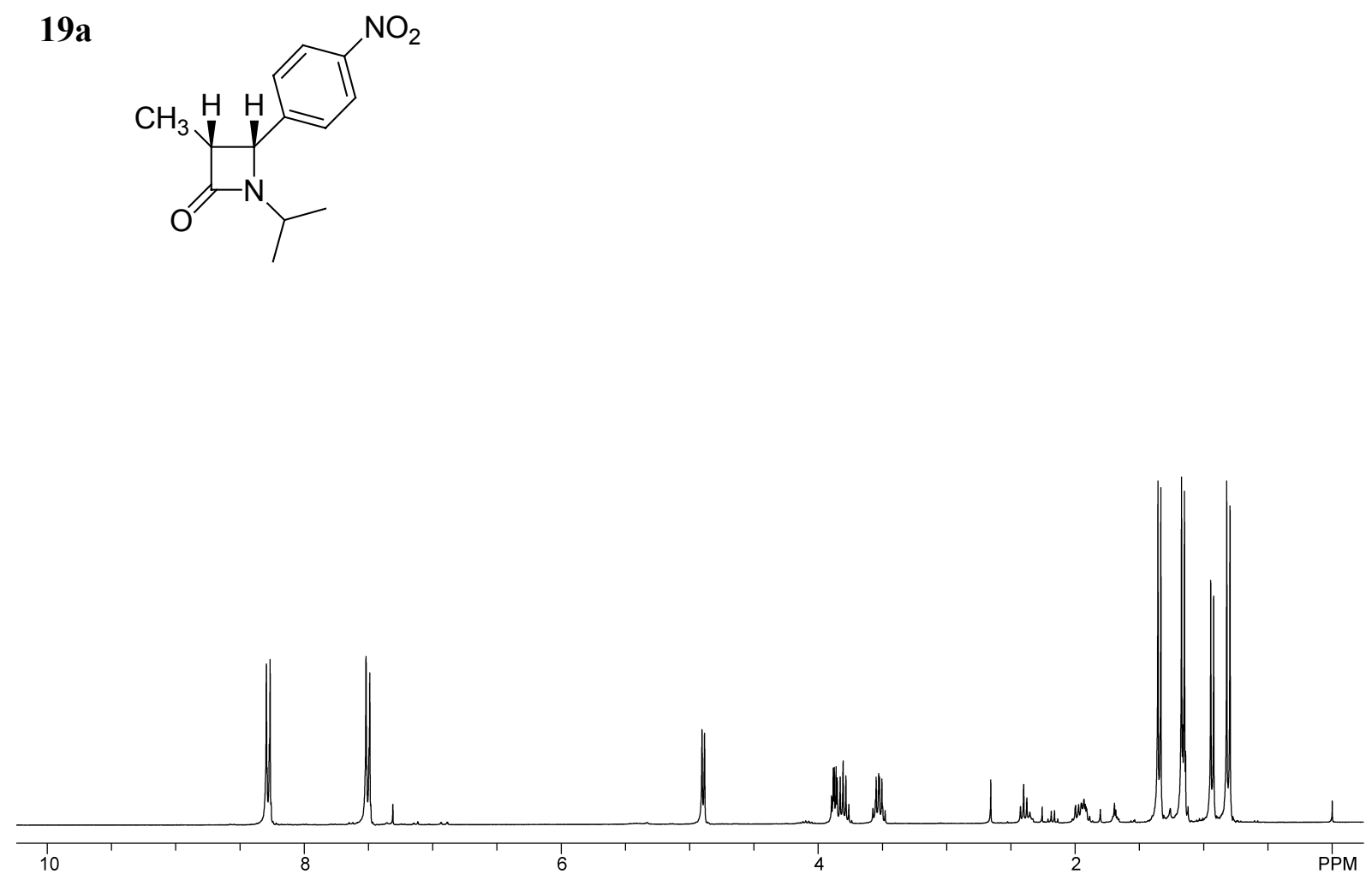

19b
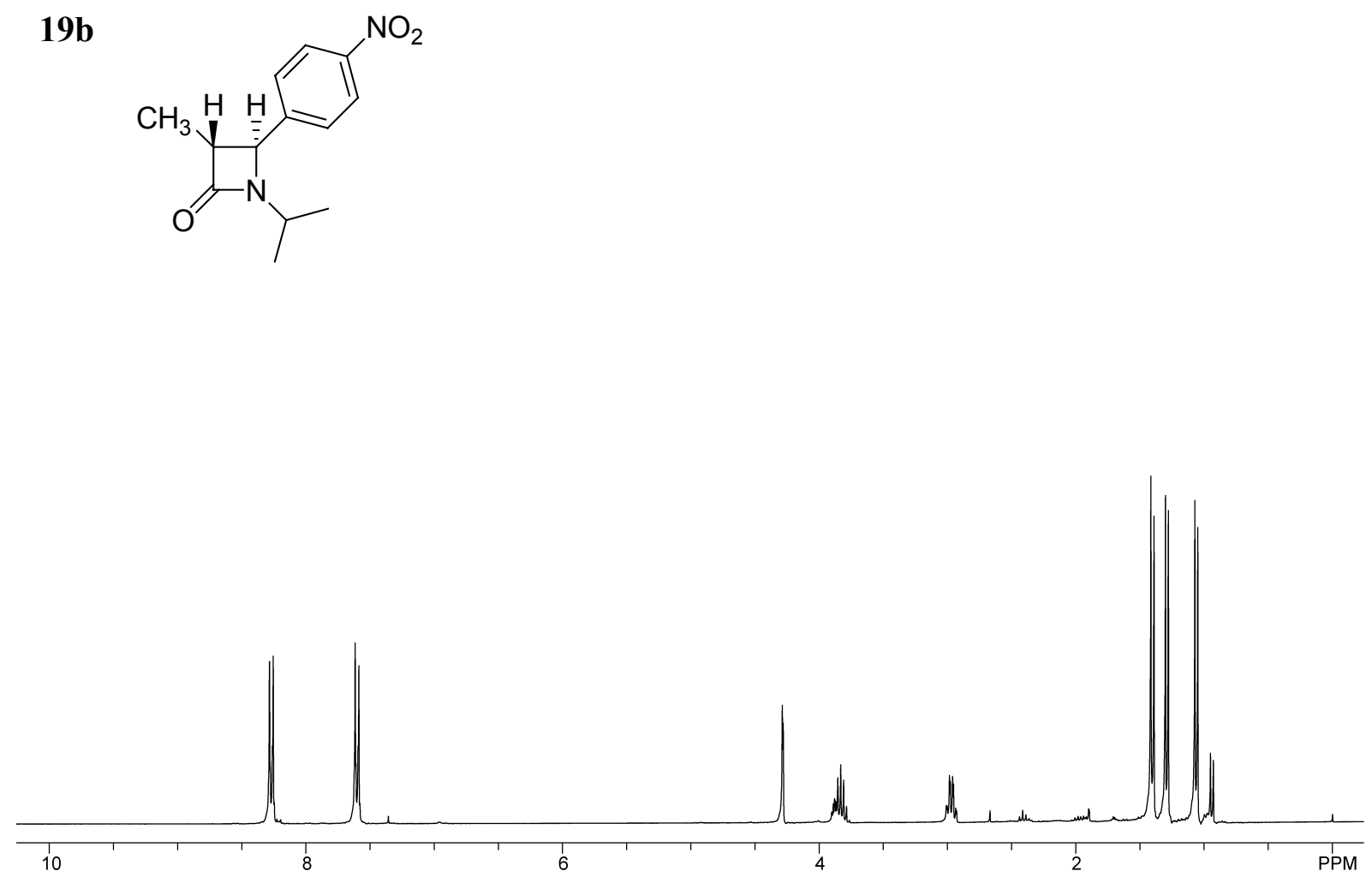

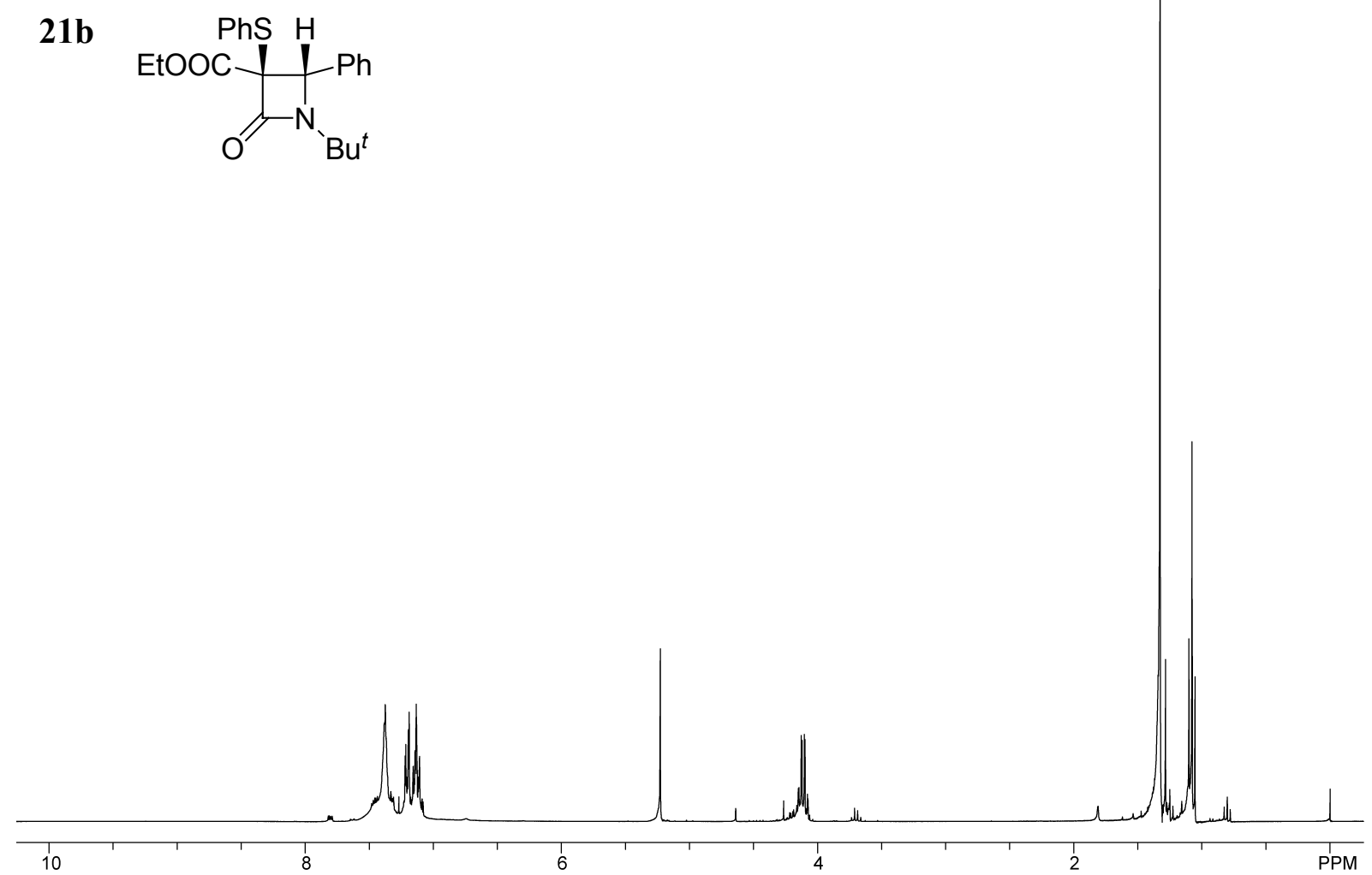

21b NOE<smiles>O=[13CH]Cc1ccccc1S</smiles><smiles>CCOC(=O)C1(S)C(=O)N(Cc2ccccc2)[C@H](c2ccccc2)[C@H]1c1ccccc1</smiles>

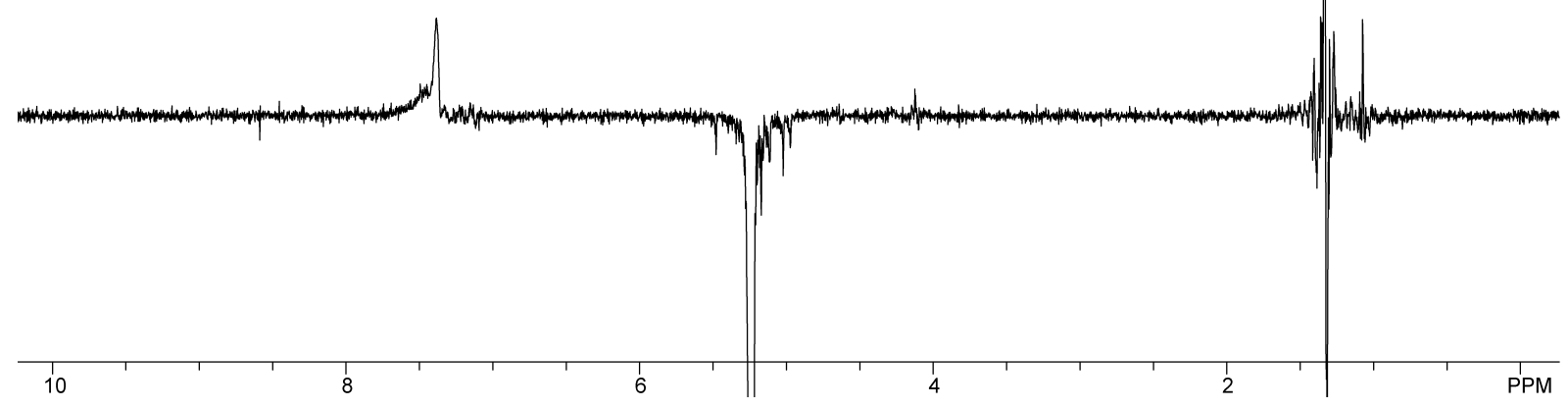

